\title{
Sonochemistry: A Powerful Way of Enhancing the Efficiency of Carbohydrate Synthesis
}

\author{
Shenglou Deng, Umesh Gangadharmath, and Cheng-Wei Tom Chang* \\ Department of Chemistry and Biochemistry, Utah State University, 0300 Old Main Hill, \\ Logan, Utah 84322-0300, U.S.A.
}

Tel: +4357973545. Fax: +4357973390. Email: chang@cc.usu.edu

\section{Supporting Information}

I. Spectroscopic Data of Products (S2 - S22)

II. ${ }^{1} \mathrm{H}$ and ${ }^{13} \mathrm{C}$ Spectra for New Compounds (S23 - S88)

III. References (S89) 


\section{Spectroscopic Data of Products}

\section{D-Galactosamine Hydrochloride (1). ${ }^{2}$}

Galactosamine Pentaacetate (2). ${ }^{1}{ }^{1} \mathrm{H}$ NMR $\left(\mathrm{CDCl}_{3}, 400 \mathrm{MHz}\right) \delta 5.71(\mathrm{~d}, J=8.8 \mathrm{~Hz}$, $1 \mathrm{H}), 5.39(\mathrm{~d}, J=3.2 \mathrm{~Hz}, 1 \mathrm{H}), 5.31(\mathrm{br}, \mathrm{NH}), 5.09$ (dd, $J=3.3,11.3 \mathrm{~Hz}, 1 \mathrm{H}), 4.45$ (dt, $J=$ 9.2, $9.2 \mathrm{~Hz}, 1 \mathrm{H}), 4.1-4.2(\mathrm{~m}, 2 \mathrm{H}), 4.03(\mathrm{t}, J=6.2 \mathrm{~Hz}, 1 \mathrm{H}), 2.18(\mathrm{~s}, 3 \mathrm{H}), 2.14(\mathrm{~s}, 3 \mathrm{H})$, $2.05(\mathrm{~s}, 3 \mathrm{H}), 2.03(\mathrm{~s}, 3 \mathrm{H}), 1.95(\mathrm{~s}, 3 \mathrm{H})$.

\section{D-Galactopyranose (3). ${ }^{2}$}

Galactose Pentaacetate (4). ${ }^{2} \mathrm{H}$ NMR $\left(\mathrm{CDCl}_{3}, 400 \mathrm{MHz}\right)(\beta$ isomer $) \delta 5.68(\mathrm{~d}, J=8.4$ $\mathrm{Hz}, 1 \mathrm{H}), 5.41(\mathrm{~d}, J=3.3 \mathrm{~Hz}, 1 \mathrm{H}), 5.31(\mathrm{t}, J=8.4 \mathrm{~Hz}, 1 \mathrm{H}), 5.07(\mathrm{dd}, J=3.4,7.0 \mathrm{~Hz}, 1 \mathrm{H})$, 4.0 - $4.2(\mathrm{~m}, 3 \mathrm{H}), 2.21(\mathrm{~s}, 3 \mathrm{H}), 2.14(\mathrm{~s}, 3 \mathrm{H}), 2.02(\mathrm{br}, 6 \mathrm{H}), 1.97(\mathrm{~s}, 3 \mathrm{H})$.

$\alpha$-D-Trehalose (5). ${ }^{2}$

2,2',3,3',4,4',6,6'-Octa-O-acetyl- $\boldsymbol{\alpha}$-D-trehalose (6). ${ }^{3}{ }^{1} \mathrm{H} \mathrm{NMR}\left(\mathrm{CDCl}_{3}, 400 \mathrm{MHz}\right) \delta 5.48$ $(\mathrm{dd}, J=9.5,10.0 \mathrm{~Hz}, 2 \mathrm{H}), 5.28(\mathrm{~d}, J=3.9 \mathrm{~Hz}, 2 \mathrm{H}), 5.0-5.1(\mathrm{~m}, 4 \mathrm{H}), 4.2-4.3(\mathrm{~m}, 2 \mathrm{H})$, $4.0-4.1(\mathrm{~m}, 4 \mathrm{H}), 2.07(\mathrm{~s}, 6 \mathrm{H}), 2.06(\mathrm{~s}, 6 \mathrm{H}), 2.04(\mathrm{~s}, 6 \mathrm{H}), 2.02(\mathrm{~s}, 6 \mathrm{H}) ;{ }^{13} \mathrm{C} \mathrm{NMR}\left(\mathrm{CDCl}_{3}\right.$, $100 \mathrm{MHz}) \delta 170.7,170.1,169.8,169.7,92.4,70.2,70.1,68.8,68.4,61.9,20.82,20.75$.

4,6:4',6'-Di-O-benzylidene- $\boldsymbol{\alpha}$-D-trehalose (7). ${ }^{4}{ }^{1} \mathrm{H}$ NMR (DMSO- $\left.d_{6}, 400 \mathrm{MHz}\right) \delta 7.3$ $7.4(\mathrm{~m}, 10 \mathrm{H}), 5.56(\mathrm{~s}, 2 \mathrm{H}), 5.24(\mathrm{~d}, J=6.0 \mathrm{~Hz}, 2 \mathrm{H}), 5.17(\mathrm{~d}, J=5.0 \mathrm{~Hz}, 2 \mathrm{H}), 4.94(\mathrm{~d}, J=$ $3.7 \mathrm{~Hz}, 2 \mathrm{H}), 4.0-4.1(\mathrm{~m}, 4 \mathrm{H}), 3.7-3.8(\mathrm{~m}, 2 \mathrm{H}), 3.7(\mathrm{t}, J=9.6 \mathrm{~Hz}, 2 \mathrm{H}), 3.4-3.5(\mathrm{~m}, 4 \mathrm{H})$. 
2,2',3,3'-Tetra- $O$-acetyl-4,6:4',6'-di-O-benzylidene- $\boldsymbol{\alpha}$-D-trehalose $\quad(8) .{ }^{5} \quad{ }^{1} \mathrm{H} \quad \mathrm{NMR}$ $\left(\mathrm{CDCl}_{3}, 400 \mathrm{MHz}\right) \delta 7.46-7.27(\mathrm{~m}, 10 \mathrm{H}), 5.64(\mathrm{t}, J=9.8 \mathrm{~Hz}, 2 \mathrm{H}), 5.51(\mathrm{~s}, 2 \mathrm{H}), 5.36(\mathrm{~d}, J$ $=4.0 \mathrm{~Hz}, 2 \mathrm{H}), 5.03(\mathrm{dd}, J=4.0,9.9 \mathrm{~Hz}, 2 \mathrm{H}), 4.18(\mathrm{dd}, J=4.9,10.4 \mathrm{~Hz}, 2 \mathrm{H}), 4.02(\mathrm{ddd}, J$ $=4.9,4.9,10.4 \mathrm{~Hz}, 2 \mathrm{H}), 3.76(\mathrm{t}, J=10.4 \mathrm{~Hz}, 2 \mathrm{H}), 3.69(\mathrm{t}, J=10.4 \mathrm{~Hz}, 2 \mathrm{H}), 2.15(\mathrm{~s}, 6 \mathrm{H})$, $2.08(\mathrm{~s}, 6 \mathrm{H}) ;{ }^{13} \mathrm{C} \mathrm{NMR}\left(\mathrm{CDCl}_{3}, 100 \mathrm{MHz}\right) \delta 170.5$ (2C), 169.6 (2C), 137.0 (2C), 129.3 (2C), 128.4 (4C), 126.4 (4C), 102.0 (2C), 93.3 (2C), 79.3 (2C), 71.1 (2C), 69.2 (2C), 68.8 (2C), $63.4(2 \mathrm{C}), 21.0(2 \mathrm{C}), 20.8(2 \mathrm{C})$.

Methyl $\alpha$-D-glucopyranoside (9). ${ }^{2}$

Methyl 4,6-O-Benzylidene- $\boldsymbol{\alpha}$-D-glucopyranoside (10). ${ }^{6}{ }^{1} \mathrm{H} \quad \mathrm{NMR} \quad\left(\mathrm{CDCl}_{3}, \quad 400\right.$ MHz) $\delta 7.52-7.27(\mathrm{~m}, 5 \mathrm{H}), 5.54(\mathrm{~s}, 1 \mathrm{H}), 4.81(\mathrm{~d}, J=3.9 \mathrm{~Hz}, 1 \mathrm{H}), 4.30(\mathrm{dd}, J=4.2,9.7$ $\mathrm{Hz}, 1 \mathrm{H}), 3.92(\mathrm{dt}, J=2.2,9.2 \mathrm{~Hz}, 1 \mathrm{H}), 3.81(\mathrm{ddd}, J=3.9,3.9,10.1 \mathrm{~Hz}, 1 \mathrm{H}), 3.75(\mathrm{t}, J=$ $10.1 \mathrm{~Hz}, 1 \mathrm{H}), 3.64(\mathrm{dt}, J=4.0,9.3 \mathrm{~Hz}, 1 \mathrm{H}), 3.50(\mathrm{t}, J=10.1 \mathrm{~Hz}, 1 \mathrm{H}), 3.47(\mathrm{~s}, 3 \mathrm{H}), 2.72$ $(\mathrm{d}, 1 \mathrm{H}, J=2.2 \mathrm{~Hz}), 2.28(\mathrm{~d}, J=9.4 \mathrm{~Hz}, 1 \mathrm{H}) ;{ }^{13} \mathrm{C} \mathrm{NMR}\left(\mathrm{CDCl}_{3}, 100 \mathrm{MHz}\right) \delta 137.3,129.5$, 128.5 (2C), 126.5 (2C), 102.2, 100.0, 81.1, 73.1, 72.1, 69.2, 62.6, 55.8.

1,2:3,4-Di- $\boldsymbol{O}$-isopropylidene- $\boldsymbol{\alpha}$-D-galactopyranose (11). ${ }^{2} \mathrm{H} \mathrm{NMR}^{1}\left(\mathrm{CDCl}_{3}, 400 \mathrm{MHz}\right)$ $\delta 5.57(\mathrm{~d}, J=5.0 \mathrm{~Hz}, 1 \mathrm{H}), 4.62(\mathrm{dd}, J=2.1,5.8 \mathrm{~Hz}, 1 \mathrm{H}), 4.34(\mathrm{dd}, J=2.3 .4 .9 \mathrm{~Hz}, 1 \mathrm{H})$, $4.27(\mathrm{~d}, J=7.9 \mathrm{~Hz}, 1 \mathrm{H}), 3.8-3.9(\mathrm{~m}, 2 \mathrm{H}), 3.7-3.8(\mathrm{~m}, 1 \mathrm{H}), 2.18$ (br, 6-OH), 1.54 (s, $3 \mathrm{H}), 1.46(\mathrm{~s}, 3 \mathrm{H}), 1.34(\mathrm{~s}, 6 \mathrm{H})$.

1,2:3,4-Di- $\boldsymbol{O}$-isopropylidene-6- $\boldsymbol{O}$-tosyl- $\boldsymbol{\alpha}$-D-galactopyranose (12). ${ }^{7}{ }^{1} \mathrm{H} \mathrm{NMR}\left(\mathrm{CDCl}_{3}\right.$, $400 \mathrm{MHz}) \delta 7.76(\mathrm{~d}, J=8.3 \mathrm{~Hz}, 2 \mathrm{H}), 7.29(\mathrm{~d}, J=8.1,2 \mathrm{H}), 5.41(\mathrm{~d}, J=5.0 \mathrm{~Hz}, 1 \mathrm{H}), 4.55$ $(\mathrm{dd}, J=2.5,7.9 \mathrm{~Hz}, 1 \mathrm{H}), 4.25(\mathrm{dd}, J=2.4,5.0 \mathrm{~Hz}, 1 \mathrm{H}), 4.1-4.2(\mathrm{~m}, 2 \mathrm{H}), 4.0-4.1(\mathrm{~m}$, 2H), $2.40(\mathrm{~s}, 3 \mathrm{H}), 1.46(\mathrm{~s}, 3 \mathrm{H}), 1.30(\mathrm{~s}, 3 \mathrm{H}), 1.28(\mathrm{~s}, 3 \mathrm{H}), 1.29(\mathrm{~s}, 3 \mathrm{H}) ;{ }^{13} \mathrm{C} \mathrm{NMR}\left(\mathrm{CDCl}_{3}\right.$, 
$100 \mathrm{MHz}) \delta 144.9,133.1,129.9$ (2C), 128.3 (2C), 109.7, 109.1, 96.3, 70.7, 70.60, 70.56, $68.4,66.1,26.1,26.0,25.1,24.5,21.7$.

Methyl 2,3-Di-O-benzyl-4,6-O-benzylidene- $\alpha$-D-glucopyranoside (13). ${ }^{8}$

Methyl 2,3-Di-O-benzyl- $\boldsymbol{\alpha}$-D-glucopyranoside (14). ${ }^{8}{ }^{1} \mathrm{H} \mathrm{NMR}\left(\mathrm{CDCl}_{3}, 400 \mathrm{MHz}\right) \delta$ 7.39-7.30 (m, 10H), $4.88(\mathrm{q}, 2 \mathrm{H}, J=11.5 \mathrm{~Hz}), 4.73(\mathrm{q}, 2 \mathrm{H}, J=12.1 \mathrm{~Hz}), 4.62(\mathrm{~d}, 1 \mathrm{H}, J=$ $3.5 \mathrm{~Hz}), 3.84-3.74(\mathrm{~m}, 2 \mathrm{H}), 3.81(\mathrm{t}, 1 \mathrm{H}, J=9.6 \mathrm{~Hz}), 3.66-3.62(\mathrm{~m}, 1 \mathrm{H}), 3.53(\mathrm{t}, 1 \mathrm{H}, J=$ $9.5 \mathrm{~Hz}), 3.50(\mathrm{~d}, 1 \mathrm{H}, J=9.5 \mathrm{~Hz}), 3.40(\mathrm{~s}, 3 \mathrm{H})$.

\section{Phenyl 2,3-Di-O-acetyl-4,6-O-benzylidene-1-thio-D-glucopyranoside (15). ${ }^{11}$}

Phenyl 2,3-Di-O-acetyl-1-thio-D-glucopyranoside (16). ${ }^{11}{ }^{1} \mathrm{H} \mathrm{NMR}\left(\mathrm{CDCl}_{3}, 400 \mathrm{MHz}\right)$ $(\beta$ anomer) $\delta 7.3-7.5(\mathrm{~m}, 5 \mathrm{H}), 5.07(\mathrm{t}, J=9.3 \mathrm{~Hz}, 1 \mathrm{H}), 4.93(\mathrm{t}, J=10.0 \mathrm{~Hz}, 1 \mathrm{H}), 4.71(\mathrm{~d}$, $J=8.0 \mathrm{~Hz}, 1 \mathrm{H}), 3.93(\mathrm{dd}, J=3.3,12.1 \mathrm{~Hz}, 1 \mathrm{H}), 3.84(\mathrm{~d}, J=4.3 \mathrm{~Hz}, 1 \mathrm{H}), 3.73(\mathrm{t}, J=9.5$ $\mathrm{Hz}, 1 \mathrm{H}), 3.4-3.5(\mathrm{~m}, 1 \mathrm{H}), 3.68(\mathrm{br}, 2 \mathrm{H}), 2.09(\mathrm{~s}, 3 \mathrm{H}), 2.08(\mathrm{~s}, 3 \mathrm{H})$.

Phenyl 3-O-Benzoyl-4,6- $O$-benzylidene-1-thio- $\beta$-D-galactopyranoside (17). ${ }^{10}$

Phenyl 3-O-Benzoyl-1-thio- $\beta$-D-galactopyranoside (18). ${ }^{1} \mathrm{H} \mathrm{NMR}\left(\mathrm{CDCl}_{3}, 400 \mathrm{MHz}\right) \delta$ $8.07(\mathrm{~d}, J=7.8 \mathrm{~Hz}, 2 \mathrm{H}), 7.5-7.6(\mathrm{~m}, 3 \mathrm{H}), 7.43(\mathrm{~d}, J=7.6 \mathrm{~Hz}, 2 \mathrm{H}), 7.3(\mathrm{~m}, 3 \mathrm{H}), 5.11$ $(\mathrm{dd}, J=2.6,9.4 \mathrm{~Hz}, 1 \mathrm{H}), 4.69(\mathrm{~d}, J=9.7 \mathrm{~Hz}, 1 \mathrm{H}), 4.30$ (s, 1H), 4.09 (dt, $J=2.5,9.6 \mathrm{~Hz}$, 1H), $3.9(\mathrm{~m}, 2 \mathrm{H}), 3.69(\mathrm{br}, 1 \mathrm{H}), 2.85(\mathrm{br}, 1 \mathrm{H}), 2.66(\mathrm{br}, 1 \mathrm{H}), 2.44(\mathrm{br}, 1 \mathrm{H}) ;{ }^{13} \mathrm{C} \mathrm{NMR}$ $\left(\mathrm{CDCl}_{3}, 100 \mathrm{MHz}\right) \delta 166.4,133.7,132.7,132.2,130.1,129.7,129.4,128.7,128.4,89.4$, 78.2, 77.1, 68.9, 67.6, 63.1; HRFAB Calcd for $\mathrm{C}_{19} \mathrm{H}_{20} \mathrm{~N}_{8} \mathrm{O}_{6} \mathrm{SNa}\left([\mathrm{M}+\mathrm{Na}]^{+}\right) \mathrm{m} / \mathrm{e} 399.0878$; measured m/e 399.0867. 
Methyl 2,3,4-Tri-O-acetyl-6-azido-6-deoxy- $\alpha$-D-glucopyranoside (19). ${ }^{8}$

Methyl 6-Azido-6-deoxy- $\boldsymbol{\alpha}$-D-glucopyranoside (20). ${ }^{8}{ }^{1} \mathrm{H} \mathrm{NMR}\left(\mathrm{CDCl}_{3}, 400 \mathrm{MHz}\right) \delta$ $4.69(\mathrm{~d}, 1 \mathrm{H}, J=3.7 \mathrm{~Hz}), 3.69-3.66(\mathrm{~m}, 1 \mathrm{H}), 3.60(\mathrm{t}, 1 \mathrm{H}, J=9.3 \mathrm{~Hz}), 3.51(\mathrm{dd}, 1 \mathrm{H}, J=2.2$, 11.0 Hz), 3.47-3.37 (m, 2H), 3.44 (s, 3H), 3.26 (t, $1 \mathrm{H}, J=9.4 \mathrm{~Hz}), 1.90(\mathrm{br}, 1 \mathrm{H}, \mathrm{OH}) ;{ }^{13} \mathrm{C}$ $\operatorname{NMR}\left(\mathrm{CDCl}_{3}, 100 \mathrm{MHz}\right) \delta 100.15,73.69,72.27,71.47,71.41,54.52,51.57$.

\section{2,2',3,3',4,4'-Hexa- $O$-benzyl-6,6'-di- $O$-trityl- $\alpha$-D-trehalose (21). ${ }^{9}$}

2,2',3,3',4,4'-Hexa-O-benzyl-o-D-trehalose (22). ${ }^{9} \mathrm{H}$ NMR $\left(\mathrm{CDCl}_{3}, 400 \mathrm{MHz}\right) \delta$ 7.36$7.26(\mathrm{~m}, 30 \mathrm{H}), 5.14(\mathrm{~d}, 2 \mathrm{H}, J=3.6 \mathrm{~Hz}), 4.89(\mathrm{q}, 4 \mathrm{H}, J=1.6 \mathrm{~Hz}), 4.83(\mathrm{q}, 4 \mathrm{H}, J=11.0$ $\mathrm{Hz}), 4.71(\mathrm{q}, 4 \mathrm{H}, J=11.8 \mathrm{~Hz}), 4.08-4.06(\mathrm{~m}, 4 \mathrm{H}), 3.60-3.58(\mathrm{~m}, 6 \mathrm{H}), 3.54(\mathrm{dd}, 2 \mathrm{H}, J=$ 3.4, $9.6 \mathrm{~Hz}), 1.54$ (broad, 2H).

Methyl 2-O-Benzoyl-4,6-O-benzylidene- $\alpha$-D-glucopyranoside (23). ${ }^{10}$

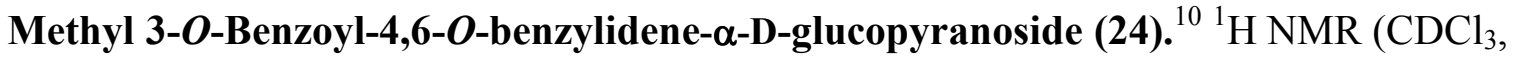
$400 \mathrm{MHz}) \delta 8.1(\mathrm{~m}, 2 \mathrm{H}), 7.6(\mathrm{~m}, 1 \mathrm{H}), 7.4-7.5(\mathrm{~m}, 4 \mathrm{H}), 7.3(\mathrm{~m}, 3 \mathrm{H}), 5.60(\mathrm{t}, J=9.6 \mathrm{~Hz}$, 1H), $5.54(\mathrm{~s}, 1 \mathrm{H}), 4.87(\mathrm{~d}, J=3.8 \mathrm{~Hz}, 1 \mathrm{H}), 4.35(\mathrm{dd}, J=4.8 \mathrm{~Hz}, J=10.3 \mathrm{~Hz}, 1 \mathrm{H}), 3.9-$ $4.0(\mathrm{~m}, 1 \mathrm{H}), 3.8-3.9(\mathrm{~m}, 3 \mathrm{H}), 3.52(\mathrm{~s}, 3 \mathrm{H}), 2.34(\mathrm{~d}, J=11.3 \mathrm{~Hz}, 1 \mathrm{H}) ;{ }^{13} \mathrm{C} \mathrm{NMR}\left(\mathrm{CDCl}_{3}\right.$, $100 \mathrm{MHz}) \delta 166.9,137.2,133.3,130.2,130.1$ (2C), 129.2, 128.5 (2C), $128.4(2 \mathrm{C}), 126.4$ (2C), 101.7, 100.5, 79.1, 73.2, 72.3, 69.2, 63.0, 55.8.

Phenyl 3-O-Decanoyl-4,6- $O$-benzylidene-1-thio- $\beta$-D-galactopyranoside (25). ${ }^{10}$ 
Phenyl 2-O-Decanoyl-4,6- $\boldsymbol{O}$-benzylidene-1-thio- $\boldsymbol{\beta}$-D-galactopyranoside $\quad(26) .{ }^{10} \quad{ }^{1} \mathrm{H}$ $\operatorname{NMR}\left(\mathrm{CDCl}_{3}, 400 \mathrm{MHz}\right) \delta 7.7(\mathrm{~m}, 2 \mathrm{H}), 7.2-7.5(\mathrm{~m}, 8 \mathrm{H}), 5.52(\mathrm{~s}, 1 \mathrm{H}), 5.08(\mathrm{t}, J=9.7$ $\mathrm{Hz}, 1 \mathrm{H}), 4.66(\mathrm{~d}, J=9.8 \mathrm{~Hz}, 1 \mathrm{H}), 4.38(\mathrm{dd}, J=1.4,12.5 \mathrm{~Hz}, 1 \mathrm{H}), 4.21(\mathrm{~d}, J=3.2 \mathrm{~Hz}$ 1H), $4.03(\mathrm{dd}, J=1.6,12.5 \mathrm{~Hz}, 1 \mathrm{H}), 3.7$ (m, 1H), 3.54 (br, 1H), 2.46 (d, $J=10.8 \mathrm{~Hz}$, $1 \mathrm{H}), 2.41(\mathrm{t}, J=7.4 \mathrm{~Hz}, 2 \mathrm{H}), 1.7(\mathrm{~m}, 2 \mathrm{H}), 1.2-1.4(\mathrm{~m}, 14 \mathrm{H}), 0.89(\mathrm{t}, J=3.5 \mathrm{~Hz}, 3 \mathrm{H})$; ${ }^{13} \mathrm{C}$ NMR $\left(\mathrm{CDCl}_{3}, 100 \mathrm{MHz}\right) \delta 173.2,137.6,133.8,131.7,130.0,129.0,128.4,128.3$, $126.8,101.7,85.1,75.8,73.0,70.1,70.0,69.3,34.6,32.1,29.7,29.5$ (2 carbons), 29.3, $25.2,22.9,14.3$.

Methyl 2-O-Acetyl-4,6- $O$-benzylidene- $\alpha$-D-glucopyranoside (27). ${ }^{10}$

Methyl 3-O-Acetyl-4,6-O-benzylidene- $\boldsymbol{\alpha}$-D-glucopyranoside (28). ${ }^{10}{ }^{1} \mathrm{H} \mathrm{NMR}\left(\mathrm{CDCl}_{3}\right.$, $400 \mathrm{MHz}) \delta 7.4-7.5(\mathrm{~m}, 2 \mathrm{H}), 7.3-7.4(\mathrm{~m}, 3 \mathrm{H}), 5.50(\mathrm{~s}, 1 \mathrm{H}), 5.34(\mathrm{dd}, J=9.7,9.7 \mathrm{~Hz}$, $1 \mathrm{H}), 4.81(\mathrm{~d}, J=3.8 \mathrm{~Hz}, 1 \mathrm{H}), 4.31(\mathrm{dd}, J=4.7,9.7 \mathrm{~Hz}, 1 \mathrm{H}), 3.88(\mathrm{ddd}, J=4.7,9.9,10.2$ $\mathrm{Hz}, 1 \mathrm{H}), 3.76(\mathrm{dd}, J=10.2,10.2 \mathrm{~Hz}, 1 \mathrm{H}), 3.67$ (m, 1H, H-2), 3.59 (dd, $J=10.2,10.2 \mathrm{~Hz}$, 1H), $3.47(\mathrm{~s}, 3 \mathrm{H}), 2.35(\mathrm{~d}, J=9.3 \mathrm{~Hz}, 1 \mathrm{H}), 2.13(\mathrm{~s}, 3 \mathrm{H}) ;{ }^{13} \mathrm{C} \mathrm{NMR}\left(\mathrm{CDCl}_{3}, 100 \mathrm{MHz}\right) \delta$ $171.3,137.2,129.3,128.5,126.4,101.8,100.3,78.9,72.5,72.0,69.1,62.9,55.8,21.3$.

Phenyl 3-O-p-Methoxybenzoyl-4,6-O-benzylidene-1-thio- $\beta$-D-galactopyranoside (29). ${ }^{10}$

Phenyl 2-O-p-Methoxybenzoyl-4,6- $O$-benzylidene-1-thio- $\beta$-D-galactopyranoside (30). ${ }^{10} 1 \mathrm{H} \mathrm{NMR}\left(\mathrm{CDCl}_{3}, 400 \mathrm{MHz}\right) \delta 8.0(\mathrm{~m}, 2 \mathrm{H}), 7.7$ (m, 2H), 7.2 - $7.5(\mathrm{~m}, 8 \mathrm{H}), 6.9(\mathrm{~m}$, 2H), $5.56(\mathrm{~s}, 1 \mathrm{H}), 5.23(\mathrm{t}, J=9.7 \mathrm{~Hz}, 1 \mathrm{H}), 4.83(\mathrm{~d}, J=9.8 \mathrm{~Hz}, 1 \mathrm{H}), 4.43(\mathrm{dd}, J=1.4$, $12.4 \mathrm{~Hz}, 1 \mathrm{H}), 4.28(\mathrm{dd}, J=0.7,3.7 \mathrm{~Hz}, 1 \mathrm{H}), 4.07(\mathrm{dd}, J=1.7,12.4 \mathrm{~Hz}, 1 \mathrm{H}), 3.9(\mathrm{~m}, 1 \mathrm{H}$, H-3), 3.89 (s, 3H), 3.63 (br, 1H), 2.65 (d, $J=10.7 \mathrm{~Hz}, 1 \mathrm{H}) ;{ }^{13} \mathrm{C} \mathrm{NMR}\left(\mathrm{CDCl}_{3}, 100 \mathrm{MHz}\right)$ 
$\delta$ 166.0, 163.9, 137.6, 134.2, 132.2, 131.3, 129.5, 129.0, 128.44, 128.40, 126.8, 122.4, $113.9,101.7,85.2,76.0,73.3,70.7,70.3,69.4,55.7$.

Phenyl 3,6-Di- $O$-pivolyl-1-thio- $\alpha$-D-mannopyranoside (31). ${ }^{10}$

Phenyl 2,6-Di-O-pivolyl-1-thio- $\boldsymbol{\alpha}$-D-mannopyranoside (32). ${ }^{10}{ }^{1} \mathrm{H}$ NMR $\left(\mathrm{CDCl}_{3}, 400\right.$ MHz) $\delta 7.50(\mathrm{~m}, 2 \mathrm{H}), 7.3(\mathrm{~m}, 3 \mathrm{H}), 5.45(\mathrm{~s}, 1 \mathrm{H}), 5.39$ (d, $J=1.1 \mathrm{~Hz}, 1 \mathrm{H}), 4.52(\mathrm{dd}, 1 \mathrm{H}, J$ $=4.3,11.8 \mathrm{~Hz}, 1 \mathrm{H}), 4.3-4.4(\mathrm{~m}, 2 \mathrm{H}), 4.04(\mathrm{dd}, J=3.3,9.4 \mathrm{~Hz}, 1 \mathrm{H}), 3.72(\mathrm{t}, J=9.5 \mathrm{~Hz}$, 1H), 1.23 (s, 9H), $1.22(\mathrm{~s}, 9 \mathrm{H}) ;{ }^{13} \mathrm{C} \mathrm{NMR}\left(\mathrm{CDCl}_{3}, 100 \mathrm{MHz}\right) \delta$ 179.8, 178.3, 133.7, 132.2, $129.3,128.1,86.7,73.3,72.0,70.9,68.3,63.9,39.3,39.2,27.4,27.3$.

$\alpha$-D-Glucose Pentaacetate (33). ${ }^{2}$

Phenyl 2,3,4,6-Tetra-O-acetyl-1-thio- $\boldsymbol{\beta}$-D-glucopyranoside (34). ${ }^{11}{ }^{1} \mathrm{H}$ NMR $\left(\mathrm{CDCl}_{3}\right.$, $400 \mathrm{MHz}) \delta 7.3-7.5(\mathrm{~m}, 5 \mathrm{H}), 5.23(\mathrm{~d}, J=3.1 \mathrm{~Hz}, 1 \mathrm{H}), 5.19(\mathrm{t}, J=9.9 \mathrm{~Hz}, 1 \mathrm{H}), 5.03(\mathrm{dd}$, $J=3.4,10.0 \mathrm{~Hz}, 1 \mathrm{H}), 4.68(\mathrm{~d}, 1 \mathrm{H}, J=9.9 \mathrm{~Hz}, 1 \mathrm{H}), 3.80(\mathrm{q}, J=6.5 \mathrm{~Hz}, 1 \mathrm{H}), 2.10(\mathrm{~s}, 3 \mathrm{H})$, $2.04(\mathrm{~s}, 3 \mathrm{H}), 1.93(\mathrm{~s}, 3 \mathrm{H}), 1.09$ (d, $J=6.5 \mathrm{~Hz}, 3 \mathrm{H})$.

$\alpha$-D-Mannose Pentaacetate (35). ${ }^{2}$

Phenyl 2,3,4,6-Tetra-O-acetyl-1-thio- $\boldsymbol{\alpha}$-D-mannopyranoside (36). ${ }^{11} \mathrm{H}$ NMR $\left(\mathrm{CDCl}_{3}\right.$, $400 \mathrm{MHz}) \delta 7.3-7.5(\mathrm{~m}, 5 \mathrm{H}), 5.51(\mathrm{t}, J=1.5 \mathrm{~Hz}, 1 \mathrm{H}), 5.50(\mathrm{br}, 1 \mathrm{H}), 5.36(\mathrm{~d}, J=9.9 \mathrm{~Hz}$, $1 \mathrm{H}), 5.53(\mathrm{t}, J=3.6 \mathrm{~Hz}, 1 \mathrm{H}), 4.5-4.6(\mathrm{~m}, 1 \mathrm{H}), 4.31(\mathrm{dd}, J=5.9,12.3 \mathrm{~Hz}, 1 \mathrm{H}), 4.12(\mathrm{dd}$, $J=2.4,12.3 \mathrm{~Hz}, 1 \mathrm{H}), 2.16$ (s, 3H), 2.08 (s, 3H), 2.06 (s, 3H), 2.03 (s, 3H).

$\alpha$-L-Rhamnose Tetraacetate (37). ${ }^{13}$ 
Phenyl 2,3,4-Tri- $\boldsymbol{O}$-acetyl-1-thio- $\boldsymbol{\alpha}$-L-rhamnopyranoside (38). ${ }^{11}{ }^{1} \mathrm{H}$ NMR $\left(\mathrm{CDCl}_{3}, 400\right.$ MHz) $\delta 7.3-7.5(\mathrm{~m}, 5 \mathrm{H}), 5.50(\mathrm{dd}, J=1.7,3.4 \mathrm{~Hz}, 1 \mathrm{H}), 5.42(\mathrm{~d}, J=1.6 \mathrm{~Hz}, 1 \mathrm{H}), 5.30$ (dd, $J=3.4,10.1 \mathrm{~Hz}, 1 \mathrm{H}), 5.15$ (t, $J=9.8 \mathrm{~Hz}, 1 \mathrm{H}), 4.3-4.4(\mathrm{~m}, 1 \mathrm{H}), 2.15(\mathrm{~s}, 3 \mathrm{H}), 2.08$ (s, 3H), $2.02(\mathrm{~s}, 3 \mathrm{H}), 1.25(\mathrm{~d}, J=6.3 \mathrm{~Hz}, 1 \mathrm{H})$.

Phenyl 2-Acetamido-2-deoxy-3,4,6-tri- $O$-acetyl-1-thio- $\beta$-D-galactopyranoside (39). ${ }^{11}$ ${ }^{1} \mathrm{H}$ NMR $\left(\mathrm{CDCl}_{3}, 400 \mathrm{MHz}\right) \delta 7.3-7.5(\mathrm{~m}, 5 \mathrm{H}), 5.57(\mathrm{~d}, J=9.0 \mathrm{~Hz}, 1 \mathrm{H}), 5.23(\mathrm{t}, J=9.7$ Hz, 1H), 5.06 (t, $J=9.8 \mathrm{~Hz}, 1 \mathrm{H}), 4.86(\mathrm{~d}, J=10.5 \mathrm{~Hz}, 1 \mathrm{H}), 4.22$ (dd, $J=5.3,12.3 \mathrm{~Hz}$, $1 \mathrm{H}), 4.17(\mathrm{dd}, J=2.6,12.2 \mathrm{~Hz}, 1 \mathrm{H}), 4.03(\mathrm{dt}, J=10.3,9.2 \mathrm{~Hz}, 1 \mathrm{H}), 4.7-4.8(\mathrm{~m}, 1 \mathrm{H})$, $2.08(\mathrm{~s}, 3 \mathrm{H}), 2.03(\mathrm{~s}, 3 \mathrm{H}), 2.02(\mathrm{~s}, 3 \mathrm{H}), 1.97$ (s, 3H).

$\alpha$-D-Galactopyranose (40). ${ }^{2}$

Phenyl 2,3,4,6-Tetra-O -acetyl-1-thio- $\boldsymbol{\beta}$-D-galactopyranoside (41). ${ }^{11}{ }^{1} \mathrm{H}$ NMR $\left(\mathrm{CDCl}_{3}\right.$, $400 \mathrm{MHz}) \delta 7.5(\mathrm{~m}, 2 \mathrm{H}), 7.2-7.3(\mathrm{~m}, 3 \mathrm{H}), 5.43(\mathrm{dd}, J=0.8,4.8 \mathrm{~Hz}, 1 \mathrm{H}), 5.3(\mathrm{t}, 1 \mathrm{H}, J=$ $10.0 \mathrm{~Hz}, 1 \mathrm{H}), 5.06$ (dd, $J=3.4,10.0 \mathrm{~Hz}, 1 \mathrm{H}), 4.73$ (d, $J=10.1 \mathrm{~Hz}, 1 \mathrm{H}), 4.20$ (dd, $J=7.0$, $11.3 \mathrm{~Hz}, 1 \mathrm{H}), 4.12$ (dd, $J=6.2,11.3 \mathrm{~Hz}, 1 \mathrm{H}), 3.94(\mathrm{dt}, J=0.8,7.0 \mathrm{~Hz}, 1 \mathrm{H}), 2.13(\mathrm{~s}, 3 \mathrm{H})$, $2.10(\mathrm{~s}, 3 \mathrm{H}), 2.05(\mathrm{~s}, 3 \mathrm{H}), 1.98(\mathrm{~s}, 3 \mathrm{H})$.

Acetyl 2,3,4-Tri- $O$-acetyl- $\alpha$-L-fucopyranoside (42). ${ }^{11}$

Phenyl 2,3,4-Tri- $\boldsymbol{O}$-acetyl-1-thio-L-fucopyranoside (43). ${ }^{11}{ }^{1} \mathrm{H}$ NMR $\left(\mathrm{CDCl}_{3}, 400\right.$ MHz) $\delta 7.3-7.5(\mathrm{~m}, 5 \mathrm{H}), 5.23(\mathrm{~d}, J=3.1 \mathrm{~Hz}, 1 \mathrm{H}), 5.19$ (t, $J=9.9 \mathrm{~Hz}, 1 \mathrm{H}), 5.03(\mathrm{dd}, J=$ 3.4, $10.0 \mathrm{~Hz}, 1 \mathrm{H}), 4.68(\mathrm{~d}, 1 \mathrm{H}, J=9.9 \mathrm{~Hz}, 1 \mathrm{H}), 3.80$ (q, $J=6.5 \mathrm{~Hz}, 1 \mathrm{H}), 2.10(\mathrm{~s}, 3 \mathrm{H})$, $2.04(\mathrm{~s}, 3 \mathrm{H}), 1.93(\mathrm{~s}, 3 \mathrm{H}), 1.09(\mathrm{~d}, J=6.5 \mathrm{~Hz}, 3 \mathrm{H})$. 
Acetyl 2-Acetamido-2-deoxy-3,4,6-tri- $O$-acetyl- $\alpha$-D-glucopyranoside (44). ${ }^{2}$

Phenyl 2-Acetamido-2-deoxy-3,4,6-tri-O-acetyl-1-thio- $\beta$-D-glucopyranoside $\quad(45){ }^{12}$ ${ }^{1} \mathrm{H} \mathrm{NMR}\left(\mathrm{CDCl}_{3}, 400 \mathrm{MHz}\right) \delta 7.3-7.5(\mathrm{~m}, 5 \mathrm{H}), 5.57(\mathrm{~d}, J=9.0 \mathrm{~Hz}, 1 \mathrm{H}), 5.23(\mathrm{t}, J=9.7$ $\mathrm{Hz}, 1 \mathrm{H}), 5.06(\mathrm{t}, J=9.8 \mathrm{~Hz}, 1 \mathrm{H}), 4.86(\mathrm{~d}, J=10.5 \mathrm{~Hz}, 1 \mathrm{H}), 4.22(\mathrm{dd}, J=5.3,12.3 \mathrm{~Hz}$, 1H), $4.17(\mathrm{dd}, J=2.6,12.2 \mathrm{~Hz}, 1 \mathrm{H}), 4.03(\mathrm{dt}, J=10.3,9.2 \mathrm{~Hz}, 1 \mathrm{H}), 4.7-4.8(\mathrm{~m}, 1 \mathrm{H})$, $2.08(\mathrm{~s}, 3 \mathrm{H}), 2.03(\mathrm{~s}, 3 \mathrm{H}), 2.02(\mathrm{~s}, 3 \mathrm{H}), 1.97(\mathrm{~s}, 3 \mathrm{H})$.

\section{2,3,4,6-Tetra- $O$-acetyl- $\alpha$-D-glucopyranosyl Bromide (46). ${ }^{14}$}

2,3,4,6-Tetra-O-acetyl- $\boldsymbol{\beta}$-D-glucopyranosyl Azide (47). ${ }^{1} \mathrm{H} \mathrm{NMR}\left(\mathrm{CDCl}_{3}, 400 \mathrm{MHz}\right) \delta$ $5.25(\mathrm{t}, J=9.5 \mathrm{~Hz}, 1 \mathrm{H}), 5.11(\mathrm{t}, J=9.7 \mathrm{~Hz}, 1 \mathrm{H}), 4.97(\mathrm{t}, J=8.9 \mathrm{~Hz}, 1 \mathrm{H}), 4.65(\mathrm{~d}, J=8.8$ $\mathrm{Hz}, 1 \mathrm{H}), 4.28(\mathrm{dd}, J=4.8,12.5 \mathrm{~Hz}, 1 \mathrm{H}), 4.18(\mathrm{dd}, J=2.2,12.4 \mathrm{~Hz}, 1 \mathrm{H}), 3.7$ - $3.8(\mathrm{~m}$, 1H), $2.11(\mathrm{~s}, 3 \mathrm{H}), 2.09$ (s, 3H), 2.04 (s, 3H), $2.02(\mathrm{~s}, 3 \mathrm{H}) ;{ }^{13} \mathrm{C} \mathrm{NMR}\left(\mathrm{CDCl}_{3}, 100 \mathrm{MHz}\right) \delta$ $170.7,170.2,169.4,169.3,88.1,74.2,72.8,70.9,68.1,61.9,20.8,20.7$ (3C); HRFAB Calcd for $\mathrm{C}_{14} \mathrm{H}_{19} \mathrm{~N}_{3} \mathrm{O}_{9} \mathrm{Na}\left([\mathrm{M}+\mathrm{Na}]^{+}\right)$m/e 396.1019; measured m/e 396.1004.

\section{2,3,4,6-Tetra- $O$-acetyl- $\alpha$-D-galactopyranosyl Bromide (48). ${ }^{14}$}

2,3,4,6-Tetra- $\boldsymbol{O}$-acetyl- $\boldsymbol{\beta}$-D-galactopyranosyl Azide (49). ${ }^{1} \mathrm{H} \mathrm{NMR}\left(\mathrm{CDCl}_{3}, 400 \mathrm{MHz}\right)$ $\delta 5.42(\mathrm{~d}, J=3.3 \mathrm{~Hz}, 1 \mathrm{H}), 5.15(\mathrm{dd}, J=8.9,10.3 \mathrm{~Hz}, 1 \mathrm{H}), 5.03(\mathrm{dd}, J=3.4,10.3 \mathrm{~Hz}, 1 \mathrm{H})$, $4.59(\mathrm{~d}, J=8.8 \mathrm{~Hz}, 1 \mathrm{H}), 4.16(\mathrm{dd}, J=3.1,6.8 \mathrm{~Hz}, 2 \mathrm{H}), 4.02(\mathrm{dt}, J=0.9,6.2 \mathrm{~Hz}, 1 \mathrm{H})$, $2.16(\mathrm{~s}, 3 \mathrm{H}), 2.08(\mathrm{~s}, 3 \mathrm{H}), 2.06(\mathrm{~s}, 3 \mathrm{H}), 1.98(\mathrm{~s}, 3 \mathrm{H}) ;{ }^{13} \mathrm{C} \mathrm{NMR}\left(\mathrm{CDCl}_{3}, 100 \mathrm{MHz}\right) \delta$ $170.5,170.3,170.1,169.5,88.5,73.1,70.9,68.3,67.1,61.4,20.81$ (2C), 20.77, 20.7; HRFAB Calcd for $\mathrm{C}_{14} \mathrm{H}_{19} \mathrm{~N}_{3} \mathrm{O}_{9} \mathrm{Na}\left([\mathrm{M}+\mathrm{Na}]^{+}\right) \mathrm{m} / \mathrm{e}$ 396.1019; measured m/e 396.1008. 
2,3,4,6-Tetra-O-acetyl- $\alpha$-D-mannopyranosyl Bromide (50). ${ }^{14}$

2,3,4,6-Tetra-O-acetyl-D-mannopyranosyl Azide (51). ${ }^{1} \mathrm{H} \mathrm{NMR}\left(\mathrm{CDCl}_{3}, 400 \mathrm{MHz}\right) \delta$ $5.51(\mathrm{~d}, J=2.6 \mathrm{~Hz}, 1 \mathrm{H}), 5.45(\mathrm{dd}, J=1.2,3.2 \mathrm{~Hz}, 1 \mathrm{H}), 5.27(\mathrm{t}, J=9.6 \mathrm{~Hz}, 1 \mathrm{H}), 5.05(\mathrm{dd}$, $J=3.2,10.0 \mathrm{~Hz}, 1 \mathrm{H}), 4.74(\mathrm{~d}, J=1.2 \mathrm{~Hz}, 1 \mathrm{H}), 4.64(\mathrm{dd}, J=2.7,3.9 \mathrm{~Hz}, 1 \mathrm{H}), 3.8(\mathrm{~m}$, 1H), $2.22(\mathrm{~s}, 3 \mathrm{H}), 2.12(\mathrm{~s}, 3 \mathrm{H}), 2.06(\mathrm{~s}, 3 \mathrm{H}), 2.00(\mathrm{~s}, 3 \mathrm{H}),{ }^{13} \mathrm{C} \mathrm{NMR}\left(\mathrm{CDCl}_{3}, 100 \mathrm{MHz}\right) \delta$ $170.77,170.75,170.0,169.7,97.7,85.3,74.9,71.2,69.4,65.6,62.4,20.9$ (2C), 20.8; HRFAB Calcd for $\mathrm{C}_{14} \mathrm{H}_{19} \mathrm{~N}_{3} \mathrm{O}_{9} \mathrm{Na}\left([\mathrm{M}+\mathrm{Na}]^{+}\right)$m/e 396.1019; measured m/e 396.1017.

2,3,4-Tri-O-acetyl- $\alpha$-D-rhamnopyranosyl Azide (52). ${ }^{14}$

2,3,4-Tri-O-acetyl- $\boldsymbol{\alpha}$-D-rhamnopyranosyl Azide (53). ${ }^{1} \mathrm{H}$ NMR $\left(\mathrm{CDCl}_{3}, 400 \mathrm{MHz}\right) \delta$ $5.44(\mathrm{~d}, J=3.1 \mathrm{~Hz}, 1 \mathrm{H}), 5.10(\mathrm{t}, J=10.0 \mathrm{~Hz}, 1 \mathrm{H}), 5.00(\mathrm{dd}, J=3.3,10.2 \mathrm{~Hz}, 1 \mathrm{H}), 4.69$ (s, $1 \mathrm{H}), 3.6-3.7(\mathrm{~m}, 1 \mathrm{H}), 2.22(\mathrm{~s}, 3 \mathrm{H}), 2.07(\mathrm{~s}, 3 \mathrm{H}), 2.00(\mathrm{~s}, 3 \mathrm{H}), 1.33(\mathrm{~d}, J=6.1 \mathrm{~Hz}, 3 \mathrm{H})$;

${ }^{13} \mathrm{C} \mathrm{NMR}\left(\mathrm{CDCl}_{3}, 100 \mathrm{MHz}\right) \delta 170.2(2 \mathrm{C}), 170.0,85.2,73.2,71.21,70.2,69.8,20.9(2 \mathrm{C})$, 20.7, 17.6; HRFAB Calcd for $\mathrm{C}_{12} \mathrm{H}_{17} \mathrm{~N}_{3} \mathrm{O}_{7} \mathrm{Na}\left([\mathrm{M}+\mathrm{Na}]^{+}\right) \mathrm{m} / \mathrm{e} 338.0964$; measured m/e 338.0954 .

6,6'-Diazido-2,2',3,3',4,4'-hexa- $O$-benzyl-6,6'-dideoxy- $\alpha$-D-trehalose (54). ${ }^{5}$

1-Octyne (55). ${ }^{2}$

2,2',3,3',4,4'-Hexa-O-benzyl-6,6'-bis(4-n-hexyl-1H-1,2,3-triazol-1-yl)- $\alpha$-D-trehalose (56). ${ }^{1} \mathrm{H} \mathrm{NMR}\left(\mathrm{CDCl}_{3}, 400 \mathrm{MHz}\right) \delta 7.1-7.4(\mathrm{~m}, 32 \mathrm{H}), 5.07$ (d, $\left.J=3.5 \mathrm{~Hz}, 2 \mathrm{H}\right), 5.00$ (d, $J=10.9 \mathrm{~Hz}, 2 \mathrm{H}), 4.90(\mathrm{dd}, J=1.5,10.4 \mathrm{~Hz}, 4 \mathrm{H}), 4.78(\mathrm{~d}, J=10.7 \mathrm{~Hz}, 2 \mathrm{H}), 4.68(\mathrm{~d}, J=$ 
$11.3 \mathrm{~Hz}, 2 \mathrm{H}), 4.55(\mathrm{~d}, J=11.9 \mathrm{~Hz}, 2 \mathrm{H}), 4.48(\mathrm{dd}, J=4.7,14.5 \mathrm{~Hz}, 2 \mathrm{H}), 4.3(\mathrm{~m}, 2 \mathrm{H})$, 4.10 (t, $J=9.3 \mathrm{~Hz}, 2 \mathrm{H}), 4.07$ (dd, $J=2.8,14.6 \mathrm{~Hz}, 2 \mathrm{H}), 3.42$ (dd, $J=3.5,9.6 \mathrm{~Hz}, 2 \mathrm{H}$ ), $3.11(\mathrm{t}, J=9.3 \mathrm{~Hz}, 2 \mathrm{H}), 2.7(\mathrm{~m}, 4 \mathrm{H}), 1.6-1.7(\mathrm{~m}, 4 \mathrm{H}), 1.3-1.4(\mathrm{~m}, 12 \mathrm{H}), 0.91(\mathrm{t}, J=$ $6.6 \mathrm{~Hz}, 6 \mathrm{H}) ;{ }^{13} \mathrm{C} \mathrm{NMR}\left(\mathrm{CDCl}_{3}, 100 \mathrm{MHz}\right) \delta 148.5$ (2C), 138.5 (2C), 138.4 (2C), 138.1 (2C), 128.70 (2C), 128.67 (4C), 128.5 (2C), 128.10 (2C), 128.05 (6C), 127.98 (4C), 127.9 (2C), 127.4 (6C), 122.3 (2C), 93.8 (2C), 81.8 (2C), 79.7 (2C), 78.1 (2C), 75.8 (2C), 75.1 (2C), 73.4 (2C), 70.0 (2C), 50.1 (2C), 31.8 (4C), 29.6 (4C), 29.2 (4C), 25.9 (2C), 22.8 (4C), 14.3 (2C); MALDI Calcd for $\mathrm{C}_{70} \mathrm{H}_{84} \mathrm{~N}_{6} \mathrm{O}_{9} \mathrm{Na}\left([\mathrm{M}+\mathrm{Na}]^{+}\right) \mathrm{m} / \mathrm{e}$ 1175.6197; measured $\mathrm{m} / \mathrm{e} 1175.6128$.

\section{1-Decyne (57). ${ }^{2}$}

\section{2,2',3,3',4,4'-Hexa-O-benzyl-6,6'-bis(4-n-octyl-1H-1,2,3-triazol-1-yl)- $\alpha$-D-trehalose}

(58). ${ }^{1} \mathrm{H}$ NMR $\left(\mathrm{CDCl}_{3}, 400 \mathrm{MHz}\right): 7.3-7.4$ (m, 30H), 7.2 (s, $\left.2 \mathrm{H}\right), 5.10(\mathrm{~d}, J=3.4 \mathrm{~Hz}$, $2 \mathrm{H}), 5.02(\mathrm{~d}, J=10.9 \mathrm{~Hz}, 2 \mathrm{H}), 4.92(\mathrm{dd}, J=2.2,10.5 \mathrm{~Hz}, 4 \mathrm{H}), 4.80(\mathrm{~d}, J=10.7 \mathrm{~Hz}, 2 \mathrm{H})$, $4.70(\mathrm{~d}, J=11.9 \mathrm{~Hz}, 2 \mathrm{H}), 4.57(\mathrm{~d}, J=11.9 \mathrm{~Hz}, 2 \mathrm{H}), 4.50(\mathrm{dd}, J=4.7,14.5 \mathrm{~Hz}, 2 \mathrm{H}), 4.3$ (m, 2H), $4.1-4.2(\mathrm{~m}, 4 \mathrm{H}), 3.45(\mathrm{dd}, J=3.4,9.6 \mathrm{~Hz}, 2 \mathrm{H}), 3.14(\mathrm{t}, J=9.3 \mathrm{~Hz}, 2 \mathrm{H}), 2.7$ (m, $4 \mathrm{H}), 1.6-1.7(\mathrm{~m}, 4 \mathrm{H}), 1.3-1.4(\mathrm{~m}, 20 \mathrm{H}), 0.94(\mathrm{t}, J=6.4 \mathrm{~Hz}, 6 \mathrm{H}) ;{ }^{13} \mathrm{C} \mathrm{NMR}\left(\mathrm{CDCl}_{3}\right.$, $100 \mathrm{MHz}) \delta 148.5$ (2C), 138.6 (2C), 138.5 (2C), 138.2 (2C), 128.73 (4C), 128.68 (6C), 128.5 (4C), 128.11 (2C), 128.08 (4C), 128.0 (2C), 127.9 (2C), 127.4 (4C), 122.3 (2C), 93.7 (2C), 81.8 (2C), 79.7 (2C), 78.1 (2C), 75.8 (2C), 75.0 (2C), 73.4 (2C), 70.0 (2C), 50.1 (2C), 32.1 (4C), 29.7 (2C), 29.6 (2C), 29.5 (4C), 25.9 (2C), 22.9 (2C), 14.3 (2C); MALDI Calcd for $\mathrm{C}_{74} \mathrm{H}_{92} \mathrm{~N}_{6} \mathrm{O}_{9} \mathrm{Na}\left([\mathrm{M}+\mathrm{Na}]^{+}\right)$m/e 1231.6823; measured m/e 1231.6856.

\section{Boc-propargylamine (59). ${ }^{2}$}


2,2',3,3',4,4'-Hexa-O-benzyl-6,6'-bis(4-tert-butyoxycarbonylmethyl-1H-1,2,3-triazol1-yl)- $\alpha$-D-trehalose (60). ${ }^{1} \mathrm{H}$ NMR $\left(\mathrm{CDCl}_{3}, 400 \mathrm{MHz}\right) \delta 7.3-7.4$ (m, 32H), 5.11 (br, 2H), $5.08(\mathrm{~d}, J=3.3 \mathrm{~Hz}, 2 \mathrm{H}), 5.00(\mathrm{~d}, J=10.9 \mathrm{~Hz}, 2 \mathrm{H}), 4.89(\mathrm{dd}, J=3.1,11.0 \mathrm{~Hz}, 4 \mathrm{H}), 4.77$ (d, $J=10.6 \mathrm{~Hz}, 2 \mathrm{H}), 4.69(\mathrm{~d}, J=11.9 \mathrm{~Hz}, 2 \mathrm{H}), 4.56(\mathrm{~d}, J=11.9 \mathrm{~Hz}, 2 \mathrm{H}), 4.49$ (dd, $J=$ 4.2, $14.6 \mathrm{~Hz}, 2 \mathrm{H}), 4.37$ (dd, $J=1.1,5.8 \mathrm{~Hz}, 4 \mathrm{H}), 4.3(\mathrm{~m}, 2 \mathrm{H}), 4.07$ (t, $J=9.2 \mathrm{~Hz}, 2 \mathrm{H})$, $3.98(\mathrm{dd}, J=2.6,14.6 \mathrm{~Hz}, 2 \mathrm{H}), 3.42(\mathrm{dd}, J=3.5,9.7 \mathrm{~Hz}, 2 \mathrm{H}), 3.06$ (dd, $J=9.1,9.8 \mathrm{~Hz}$, 2H), 1.46 (s, 18H); $\left.{ }^{13} \mathrm{C} \mathrm{NMR} \mathrm{(} \mathrm{CDCl}_{3}, 100 \mathrm{MHz}\right) \delta 156.0$ (2C), 145.3 (2C), 138.5 (2C), 138.4 (2C), 138.2 (2C), 128.8 (6C), 128.7 (8C), 128.5 (4C), 128.1 (2C), 128.0 (4C), 127.9 (2C), 127.4 (4C), 123.7 (2C), 94.4 (2C), 81.8 (2C), 79.8 (2C), 79.7 (2C), 77.8 (2C), 75.8 (2C), 75.2 (2C), 73.4 (2C), 69.7 (2C), 50.0 (2C), 36.4 (2C), 28.6 (6C); MALDI Calcd for $\mathrm{C}_{70} \mathrm{H}_{82} \mathrm{~N}_{8} \mathrm{O}_{13} \mathrm{Na}\left([\mathrm{M}+\mathrm{Na}]^{+}\right)$m/e 1265.5894; measured m/e 1265.5955.

\section{6'-Azidohexyl 2,3,4,6-tetra-O-acetyl- $\beta$-D-glucopyranoside (61). ${ }^{15}$}

\section{6-(4-tert-Butyoxycarbonylmethyl-1H-1,2,3-triazol-1-yl)hexyl $\quad 2,3,4,6$-tetra- $O$-acetyl-} 及-D-glucopyranoside (62). ${ }^{1} \mathrm{H} \mathrm{NMR}\left(\mathrm{CDCl}_{3}, 400 \mathrm{MHz}\right) \delta 7.43$ (s, 1H), 5.13 (t, $J=9.5$ Hz, 1H), 5.00 (t, $J=9.8 \mathrm{~Hz}, 1 \mathrm{H}), 4.89$ (dd, $J=8.0,9.5 \mathrm{~Hz}, 1 \mathrm{H}), 4.40$ (d, $J=7.9 \mathrm{~Hz}, 1 \mathrm{H})$, $4.32(\mathrm{~d}, J=5.9 \mathrm{~Hz}, 2 \mathrm{H}), 4.25$ (t, $J=7.1 \mathrm{~Hz}, 2 \mathrm{H}), 4.19(\mathrm{dd}, J=4.8,12.3 \mathrm{~Hz}, 1 \mathrm{H}), 4.07$ (dd, $J=2.4,12.3 \mathrm{~Hz}, 1 \mathrm{H}), 3.8(\mathrm{~m}, 1 \mathrm{H}), 3.6(\mathrm{~m}, 1 \mathrm{H}), 3.4(\mathrm{~m}, 1 \mathrm{H}), 2.01(\mathrm{~s}, 3 \mathrm{H}), 1.95(\mathrm{~s}$, 6H), 1.93 (s, 3H), $1.8-1.9(\mathrm{~m}, 2 \mathrm{H}), 1.5-1.6(\mathrm{~m}, 2 \mathrm{H}), 1.37$ (s, 9H), $1.2-1.3(\mathrm{~m}, 2 \mathrm{H})$; ${ }^{13} \mathrm{C} \mathrm{NMR}\left(\mathrm{CDCl}_{3}, 100 \mathrm{MHz}\right) \delta 170.8,170.4,169.6,169.5,156.1,145.7,121.9,101.0$, 79.8, 73.0, 72.0, 71.5, 69.6, 68.7, 62.1, 50.3, 36.4, 30.0, 29.9, 28.9, 28.6 (3C), 23.1, 20.9, 20.81, 20.76; MALDI Calcd for $\mathrm{C}_{27} \mathrm{H}_{42} \mathrm{~N}_{4} \mathrm{NaO}_{12}\left([\mathrm{M}+\mathrm{Na}]^{+}\right) \mathrm{m} / \mathrm{e}$ 637.2697; measured m/e 637. 
6-Azidohexyl 2,3,4-tri- $\boldsymbol{O}$-acetyl- $\boldsymbol{\alpha}$-L-rhamnopyranoside (63). ${ }^{1} \mathrm{H}$ NMR $\left(\mathrm{CDCl}_{3}, 400\right.$ MHz) $\delta 5.27(\mathrm{dd}, J=3.5,10.1 \mathrm{~Hz}, 1 \mathrm{H}), 5.21(\mathrm{dd}, J=1.6,3.3 \mathrm{~Hz}, 1 \mathrm{H}), 5.04(\mathrm{t}, J=9.9 \mathrm{~Hz}$, 1H), $4.71(\mathrm{~s}, 1 \mathrm{H}), 3.8-3.9(\mathrm{~m}, 1 \mathrm{H}), 3.6-3.7(\mathrm{~m}, 1 \mathrm{H}), 3.4-3.5(\mathrm{~m}, 1 \mathrm{H}), 3.26(\mathrm{t}, J=6.9$ Hz, 2H), 2.15 (s, 3H), 2.05 (s, 3H), 1.99 (s, 3H), 1.6 (m, 4H), 1.4 (m, 4H), 1.23 (d, $J=6.4$ $\mathrm{Hz}, 3 \mathrm{H}) ;{ }^{13} \mathrm{C} \mathrm{NMR}\left(\mathrm{CDCl}_{3}, 100 \mathrm{MHz}\right) \delta 170.3,170.1$ (2 carbons), 97.6, 71.4, 70.2, 69.4, 68.1, 66.5, 51.5, 29.3, 28.9, 26.6, 25.9, 21.1, 20.93, 20.86, 19.6; HRFAB Calcd for $\mathrm{C}_{18} \mathrm{H}_{30} \mathrm{~N}_{3} \mathrm{O}_{8} \mathrm{Na}\left([\mathrm{MH}]^{+}\right) \mathrm{m} / \mathrm{e} 416.2033$; measured m/e 416.2042.

6-(4-n-Hexyl-1H-1,2,3-triazol-1-yl)hexyl 2,3,4-tri- $O$-acetyl- $\alpha$-L-rhamnopyranoside

(64). ${ }^{1} \mathrm{H} \mathrm{NMR}\left(\mathrm{CDCl}_{3}, 400 \mathrm{MHz}\right) \delta 7.24(\mathrm{~s}, 1 \mathrm{H}), 5.23(\mathrm{dd}, J=3.4,10.0 \mathrm{~Hz}, 1 \mathrm{H}), 5.16$ (br, 1H), 5.01 (t, $J=9.9 \mathrm{~Hz}, 1 \mathrm{H}), 4.65$ (br, 1H), 4.27 (t, $J=7.2 \mathrm{~Hz}, 2 \mathrm{H}), 3.8(\mathrm{~m}, 1 \mathrm{H}), 3.6-$ $3.7(\mathrm{~m}, 1 \mathrm{H}), 3.3-3.4(\mathrm{~m}, 1 \mathrm{H}), 2.66(\mathrm{t}, J=7.6 \mathrm{~Hz}, 2 \mathrm{H}), 2.10(\mathrm{~s}, 3 \mathrm{H}), 2.00(\mathrm{~s}, 3 \mathrm{H}), 1.94(\mathrm{~s}$, $3 \mathrm{H}), 1.8-1.9(\mathrm{~m}, 2 \mathrm{H}), 1.6-1.7(\mathrm{~m}, 2 \mathrm{H}), 1.5-1.6(\mathrm{~m}, 2 \mathrm{H}), 1.2-1.4(\mathrm{~m}, 10 \mathrm{H}), 1.17$ (d, $J$ $=6.3 \mathrm{~Hz}, 3 \mathrm{H}), 0.83(\mathrm{t}, J=5.9 \mathrm{~Hz}, 3 \mathrm{H}) ;{ }^{13} \mathrm{C} \mathrm{NMR}\left(\mathrm{CDCl}_{3}, 100 \mathrm{MHz}\right) \delta 170.3,170.12$, $170.10,148.6,120.6,97.56,71.3,70.1,69.4,68.1,66.5,50.2,30.7,30.4,29.6,29.2,29.1$, 26.5, 25.85, 25.8, 22.7, 21.0, 21.9, 20.9, 17.6, 14.2; HRFAB Calcd for $\mathrm{C}_{26} \mathrm{H}_{44} \mathrm{~N}_{3} \mathrm{O}_{8}$ $\left([\mathrm{MH}]^{+}\right) \mathrm{m} / \mathrm{e} 526.3128 ;$ measured $\mathrm{m} / \mathrm{e} 526.3137$.

6-(4-n-Octyl-1H-1,2,3-triazol-1-yl)hexyl 2,3,4-tri- $O$-acetyl- $\alpha$-L-rhamnopyranoside (65). ${ }^{1} \mathrm{H}$ NMR ( $\left.\mathrm{CDCl}_{3}, 400 \mathrm{MHz}\right): \delta 7.26(\mathrm{~s}, 1 \mathrm{H}) 5.28(\mathrm{dd}, J=3.3,10.1 \mathrm{~Hz}, 1 \mathrm{H}), 5.21(\mathrm{br}$, $1 \mathrm{H}), 5.06(\mathrm{t}, J=9.9 \mathrm{~Hz}, 1 \mathrm{H}), 4.70(\mathrm{br}, 1 \mathrm{H}), 4.32(\mathrm{t}, J=7.2 \mathrm{~Hz}, 2 \mathrm{H}), 3.8-3.9(\mathrm{~m}, 1 \mathrm{H})$, $3.6-3.7(\mathrm{~m}, 1 \mathrm{H}), 3.4(\mathrm{~m}, 1 \mathrm{H}), 2.70(\mathrm{t}, J=7.7 \mathrm{~Hz}, 2 \mathrm{H}), 2.15(\mathrm{~s}, 3 \mathrm{H}), 2.05(\mathrm{~s}, 3 \mathrm{H}), 1.98(\mathrm{~s}$, $3 \mathrm{H}), 1.8-1.9(\mathrm{~m}, 2 \mathrm{H}), 1.6-1.7(\mathrm{~m}, 2 \mathrm{H}), 1.6(\mathrm{~m}, 2 \mathrm{H}), 1.3-1.4(\mathrm{~m}, 14 \mathrm{H}), 1.21(\mathrm{~d}, J=$ $6.2 \mathrm{~Hz}, 3 \mathrm{H}), 0.87$ (t, $J=5.8 \mathrm{~Hz}, 3 \mathrm{H}) ;{ }^{13} \mathrm{C} \mathrm{NMR}\left(\mathrm{CDCl}_{3}, 100 \mathrm{MHz}\right) \delta 170.3,170.13$, $170.11,148.6,120.6,97.6,71.3,70.2,69.4,68.1,66.5,50.2,31.0,30.4,29.7,29.5,29.43$, 
29.35, 29.2, 26.5, 25.9, 25.8, 22.8, 21.1, 20.93, 20.86, 17.6, 14.2; HRFAB Calcd for $\mathrm{C}_{28} \mathrm{H}_{48} \mathrm{~N}_{3} \mathrm{O}_{8}\left([\mathrm{MH}]^{+}\right)$m/e 554.3441; measured m/e 554.3447.

6-(4-tert-Butyoxycarbonylmethyl-1H-1,2,3-triazol-1-yl)hexyl $\quad 2,3,4-$ tri- $O$-acetyl- $\alpha$-Lrhamnopyranoside (66). ${ }^{1} \mathrm{H}$ NMR $\left(\mathrm{CDCl}_{3}, 400 \mathrm{MHz}\right): \delta 7.26$ (s, $\left.1 \mathrm{H}\right) 5.28$ (dd, $J=3.3$, $10.1 \mathrm{~Hz}, 1 \mathrm{H}), 5.21(\mathrm{br}, 1 \mathrm{H}), 5.06(\mathrm{t}, J=9.9 \mathrm{~Hz}, 1 \mathrm{H}), 4.70(\mathrm{br}, 1 \mathrm{H}), 4.32(\mathrm{t}, J=7.2 \mathrm{~Hz}$, 2H), $3.8-3.9(\mathrm{~m}, 1 \mathrm{H}), 3.6-3.7(\mathrm{~m}, 1 \mathrm{H}), 3.4(\mathrm{~m}, 1 \mathrm{H}), 2.70(\mathrm{t}, J=7.7 \mathrm{~Hz}, 2 \mathrm{H}), 2.15(\mathrm{~s}$, 3H), 2.05 (s, 3H), $1.98(\mathrm{~s}, 3 \mathrm{H}), 1.8-1.9(\mathrm{~m}, 2 \mathrm{H}), 1.6-1.7(\mathrm{~m}, 2 \mathrm{H}), 1.6(\mathrm{~m}, 2 \mathrm{H}), 1.3-$ $1.4(\mathrm{~m}, 14 \mathrm{H}), 1.21(\mathrm{~d}, J=6.2 \mathrm{~Hz}, 3 \mathrm{H}), 0.87(\mathrm{t}, J=5.8 \mathrm{~Hz}, 3 \mathrm{H}) ;{ }^{13} \mathrm{C} \mathrm{NMR}\left(\mathrm{CDCl}_{3}, 100\right.$ MHz) $\delta 170.3,170.13,170.11,148.6,120.6,97.6,71.3,70.2,69.4,68.1,66.5,50.2,31.0$, $30.4,29.7,29.5,29.43,29.35,29.2,26.5,25.9,25.8,22.8,21.1,20.93,20.86,17.6,14.2$; HRFAB Calcd for $\mathrm{C}_{28} \mathrm{H}_{48} \mathrm{~N}_{3} \mathrm{O}_{8}\left([\mathrm{MH}]^{+}\right)$m/e 554.3441; measured m/e 554.3447.

\section{Methyl 6-Azido-2,3,4-tri- $O$-acetyl-6-deoxy- $\alpha$-D-gluco-pyranoside (67). ${ }^{8}$}

\section{Methyl 2,3,4-Tri- $O$-acetyl-6-deoxy-6-(4-n-hexyl-1H-1,2,3-triazol-1-yl)- $\alpha$-D-gluco-} pyranoside (68). ${ }^{1} \mathrm{H} \mathrm{NMR}\left(\mathrm{CDCl}_{3}, 400 \mathrm{MHz}\right) \delta 7.38(\mathrm{~s}, 1 \mathrm{H}), 5.47(\mathrm{t}, J=9.6 \mathrm{~Hz}, 1 \mathrm{H})$, 4.89 (t, $J=3.5 \mathrm{~Hz}, 1 \mathrm{H}), 4.85$ (d, $J=2.8 \mathrm{~Hz}, 1 \mathrm{H}), 4.83$ (dd, $J=3.0,6.5 \mathrm{~Hz}, 1 \mathrm{H}), 4.55$ (dd, $J=2.1,14.2 \mathrm{~Hz}, 1 \mathrm{H}), 4.30(\mathrm{dd}, J=8.7,14.2 \mathrm{~Hz}, 1 \mathrm{H}), 4.18(\mathrm{dt}, J=2.0,8.2 \mathrm{~Hz}, 1 \mathrm{H}), 3.11$ (s, 3H), $2.71(\mathrm{t}, J=7.0 \mathrm{~Hz}, 2 \mathrm{H}), 2.09$ (s, 3H), $2.06(\mathrm{~s}, 3 \mathrm{H}), 2.00(\mathrm{~s}, 3 \mathrm{H}), 1.6-1.7(\mathrm{~m}, 2 \mathrm{H})$, $1.3(\mathrm{~m}, 6 \mathrm{H}), 0.87(\mathrm{t}, J=6.0 \mathrm{~Hz}, 3 \mathrm{H}) ;{ }^{13} \mathrm{C} \mathrm{NMR}\left(\mathrm{CDCl}_{3}, 100 \mathrm{MHz}\right) \delta 170.3,170.1(2 \mathrm{C})$, $148.7,122.3,96.8,71.0,70.3,70.0,68.2,55.5,50.8,31.7,29.9,29.6,29.0,25.8,22.7$, 20.9, 20.8, 14.2; HRFAB Calcd for $\mathrm{C}_{21} \mathrm{H}_{34} \mathrm{~N}_{3} \mathrm{O}_{8}\left([\mathrm{MH}]^{+}\right)$m/e 456.2346; measured m/e 456.2338 . 
Methyl 2,3,4-Tri-O-acetyl-6-deoxy-6-(4-n-octyl-1H-1,2,3-triazol-1-yl)- $\alpha$-D-glucopyranoside (69). ${ }^{1} \mathrm{H} \mathrm{NMR}\left(\mathrm{CDCl}_{3}, 400 \mathrm{MHz}\right) \delta 7.38(\mathrm{~s}, 1 \mathrm{H}), 5.48(\mathrm{dd}, J=9.5,9.9 \mathrm{~Hz}$, $1 \mathrm{H}), 4.89(\mathrm{t}, J=3.7 \mathrm{~Hz}, 1 \mathrm{H}), 4.85(\mathrm{~d}, J=2.9 \mathrm{~Hz}, 1 \mathrm{H}), 4.83(\mathrm{dd}, J=2.6,6.4 \mathrm{~Hz}, 1 \mathrm{H})$, $4.56(\mathrm{dd}, J=2.4,14.2 \mathrm{~Hz}, 1 \mathrm{H}), 4.29(\mathrm{dd}, J=8.7,14.3 \mathrm{~Hz}, 1 \mathrm{H}), 4.1-4.2(\mathrm{~m}, 1 \mathrm{H}), 3.1(\mathrm{~s}$, 3H), $2.70(\mathrm{dd}, J=6.8,8.1 \mathrm{~Hz}, 2 \mathrm{H}), 2.09(\mathrm{~s}, 3 \mathrm{H}), 2.06(\mathrm{~s}, 3 \mathrm{H}), 2.00(\mathrm{~s}, 3 \mathrm{H}), 1.6-1.7(\mathrm{~m}$, 2H), $1.3(\mathrm{~m}, 10 \mathrm{H}), 1.87(\mathrm{t}, J=6.6 \mathrm{~Hz}, 3 \mathrm{H}) ;{ }^{13} \mathrm{C} \mathrm{NMR}\left(\mathrm{CDCl}_{3}, 100 \mathrm{MHz}\right) \delta 170.3,170.1$ (2C), 148.7, 122.3, 96.8, 71.0, 70.3, 70.0, 68.2, 55.5, 50.8, 32.0, 29.7, 29.5, 29.4 (2C), 25.8, 22.8, 20.9 (2C), 20.8, 14.3; HRFAB Calcd for $\mathrm{C}_{23} \mathrm{H}_{38} \mathrm{~N}_{3} \mathrm{O}_{8}\left([\mathrm{MH}]^{+}\right) \mathrm{m} / \mathrm{e} 484.2659$; measured m/e 484.2645.

Methyl 2,3,4-Tri-O-acetyl-6-deoxy-6-(4-n-tert-butyoxycarbonylmethyl -1H-1,2,3triazol-1-yl)- $\boldsymbol{\alpha}$-D-glucopyranoside (70). ${ }^{1} \mathrm{H} \mathrm{NMR}\left(\mathrm{CDCl}_{3}, 400 \mathrm{MHz}\right) \delta 7.64(\mathrm{~s}, 1 \mathrm{H})$, $5.48(\mathrm{t}, J=9.8 \mathrm{~Hz}, 1 \mathrm{H}), 5.11(\mathrm{br}, 1 \mathrm{H}), 4.90(\mathrm{~d}, J=3.6 \mathrm{~Hz}, 1 \mathrm{H}), 4.88(\mathrm{dd}, J=4.2,5.6 \mathrm{~Hz}$, $1 \mathrm{H}), 4.83(\mathrm{dd}, J=1.0,5.6 \mathrm{~Hz}, 1 \mathrm{H}), 4.57(\mathrm{dd}, J=2.3,14.4 \mathrm{~Hz}, 1 \mathrm{H}), 4.39$ (d, $J=6.1 \mathrm{~Hz}$, 2H), $4.35(\mathrm{dd}, J=9.5,14.3 \mathrm{~Hz}, 1 \mathrm{H}), 4.20(\mathrm{~m}, 1 \mathrm{H}), 3.14(\mathrm{~s}, 3 \mathrm{H}), 2.11(\mathrm{~s}, 3 \mathrm{H}), 2.07$ (s, 3H), $2.01(\mathrm{~s}, 3 \mathrm{H}), 1.44(\mathrm{~s}, 9 \mathrm{H}) ;{ }^{13} \mathrm{C} \mathrm{NMR}\left(\mathrm{CDCl}_{3}, 100 \mathrm{MHz}\right) \delta 170.3,170.1,170.0,156.0$, $145.8,123.6,96.8,77.4,70.9,70.2,70.0,68.1,55.7,50.9,36.3,28.6(3 \mathrm{C}), 20.9$ (3C), HRFAB Calcd for $\mathrm{C}_{21} \mathrm{H}_{33} \mathrm{~N}_{4} \mathrm{O}_{10}\left([\mathrm{MH}]^{+}\right)$m/e 501.2197; measured m/e 501.2221.

\section{Phenyl 2,3-Di- $O$-benzoyl-4,6-O-benzylidene-1-thio- $\beta$-D-glucopyranoside (71). ${ }^{11}$}

Phenyl 2,3-Di- $\boldsymbol{O}$-benzoyl-4- $\boldsymbol{O}$-benzyl-1-thio- $\boldsymbol{\beta}$-D-glucopyranoside (72). ${ }^{1} \mathrm{H} \quad \mathrm{NMR}$ $\left(\mathrm{CDCl}_{3}, 400 \mathrm{MHz}\right) \delta 7.96(\mathrm{~d}, J=7.2 \mathrm{~Hz}, 2 \mathrm{H}), 7.92(\mathrm{~d}, J=7.2 \mathrm{~Hz}, 2 \mathrm{H}), 7.4-7.6(\mathrm{~m}, 4 \mathrm{H})$, $7.2-7.4(\mathrm{~m}, 8 \mathrm{H}), 7.1-7.2(\mathrm{~m}, 4 \mathrm{H}), 5.76(\mathrm{t}, J=9.4 \mathrm{~Hz}, 1 \mathrm{H}), 5.37(\mathrm{t}, J=9.9 \mathrm{~Hz}, 1 \mathrm{H})$, $4.99(\mathrm{~d}, J=9.9 \mathrm{~Hz}, 1 \mathrm{H}), 4.60(\mathrm{~s}, 2 \mathrm{H}), 3.99(\mathrm{dd}, J=2.0,12.2 \mathrm{~Hz}, 1 \mathrm{H}), 3.93(\mathrm{t}, J=9.6 \mathrm{~Hz}$, $1 \mathrm{H}), 3.81(\mathrm{dd}, J=3.9,12.3 \mathrm{~Hz}, 2 \mathrm{H}), 3.6-3.7(\mathrm{~m}, 1 \mathrm{H}), 1.95(\mathrm{br}, 1 \mathrm{H}) ;{ }^{13} \mathrm{C} \mathrm{NMR}\left(\mathrm{CDCl}_{3}\right.$, 
$100 \mathrm{MHz}) \delta 165.9,165.5,137.3,133.44,133.38,132.9,132.5,130.1,129.9,129.6,129.5$, $129.2,128.6,128.42,128.37,128.2,86.4,79.8,76.5,75.6,75.1,71.1,62.0$; HRFAB Calcd for $\mathrm{C}_{33} \mathrm{H}_{30} \mathrm{O}_{7} \mathrm{SNa}\left([\mathrm{M}+\mathrm{Na}]^{+}\right)$m/e 593.1610; measured m/e 593.1631.

\section{Phenyl 4,6-O-benzylidene-1-thio- $\beta$-D-glucopyranoside (73). ${ }^{11}$}

Phenyl 4-O-benzyl-1-thio- $\beta$-D-glucopyranoside (74). ${ }^{1} \mathrm{H} \mathrm{NMR}\left(\mathrm{CDCl}_{3}, 400 \mathrm{MHz}\right) \delta 7.5$ (m, 2H), 7.3 - $7.4(\mathrm{~m}, 8 \mathrm{H}), 4.85$ (d, $J=11.4 \mathrm{~Hz}, 1 \mathrm{H}), 4.73$ (d, $J=11.4 \mathrm{~Hz}, 1 \mathrm{H}), 4.57$ (d, $J$ $=9.8 \mathrm{~Hz}, 1 \mathrm{H}), 3.93(\mathrm{dd}, J=3.0,12.0 \mathrm{~Hz}, 1 \mathrm{H}), 3.7-3.8(\mathrm{~m}, 2 \mathrm{H}), 3.4-3.5(\mathrm{~m}, 2 \mathrm{H}), 3.36$ (dt, $J=1.4,10.1 \mathrm{~Hz}, 1 \mathrm{H}), 2.65(\mathrm{~d}, J=1.8 \mathrm{~Hz}, 1 \mathrm{H}), 2.57$ (d, $J=2.2 \mathrm{~Hz}, 1 \mathrm{H}), 1.94(\mathrm{t}, J=$ $6.6 \mathrm{~Hz}, 1 \mathrm{H}) ;{ }^{13} \mathrm{C} \mathrm{NMR}\left(\mathrm{CDCl}_{3}, 100 \mathrm{MHz}\right) \delta 138.2,132.9,131.9,129.4,128.8,128.5$, 128.3, 88.1, 79.6, 78.3, 77.1, 75.0, 72.6, 62.4; HRFAB Calcd for $\mathrm{C}_{19} \mathrm{H}_{23} \mathrm{O}_{5} \mathrm{SNa}\left([\mathrm{M}+\mathrm{H}]^{+}\right)$ $\mathrm{m} / \mathrm{e} 363.1266$; measured m/e 363.1250.

Methyl 2,3,4-Tri- $\boldsymbol{O}$-benzyl- $\boldsymbol{\alpha}$-D-glucopyranoside (75). ${ }^{8}{ }^{1} \mathrm{H} \mathrm{NMR}\left(\mathrm{CDCl}_{3}, 400 \mathrm{MHz}\right) \delta$ $7.3-7.4(\mathrm{~m}, 15 \mathrm{H}), 5.03(\mathrm{~d}, J=11.0 \mathrm{~Hz}, 1 \mathrm{H}), 4.92(\mathrm{~d}, J=11.1 \mathrm{~Hz}, 1 \mathrm{H}), 4.88$ (d, $J=11.0$ Hz, 1H), $4.83(\mathrm{~d}, J=12.1 \mathrm{~Hz}, 1 \mathrm{H}), 4.70(\mathrm{~d}, J=12.1 \mathrm{~Hz}, 1 \mathrm{H}), 4.68(\mathrm{~d}, J=11.1 \mathrm{~Hz}, 1 \mathrm{H})$, $4.62(\mathrm{~d}, J=3.5 \mathrm{~Hz}, 1 \mathrm{H}), 4.06(\mathrm{t}, J=9.2 \mathrm{~Hz}, 1 \mathrm{H}), 3.81(\mathrm{dd}, J=2.2,11.8 \mathrm{~Hz}, 1 \mathrm{H}), 3.73$ (dd, $J=3.4,11.8 \mathrm{~Hz}, 1 \mathrm{H}), 3.7(\mathrm{~m}, 1 \mathrm{H}), 3.57(\mathrm{t}, J=9.2 \mathrm{~Hz}, 1 \mathrm{H}), 3.55(\mathrm{dd}, J=3.6,9.7 \mathrm{~Hz}$, 1H), 3.40 (s, 3H), 1.85 (br, $1 \mathrm{H}) ;{ }^{13} \mathrm{C} \mathrm{NMR}\left(\mathrm{CDCl}_{3}, 100 \mathrm{MHz}\right) \delta$ 139.0, 138.44, 138.39, $128.7,128.6,128.3,128.24,128.18,128.1,127.8,98.4,82.2,80.3,77.1,76.0,75.3,73.6$, $71.0,68.1,55.4$.

Methyl 3- $O$-Benzyl-4,6- $O$-benzylidene- $\alpha$-D-glucopyranoside (76). ${ }^{16}$ 
Methyl 3,4-Di-O-benzyl- $\alpha$-D-glucopyranoside (77). ${ }^{1} \mathrm{H}$ NMR $\left(\mathrm{CDCl}_{3}, 400 \mathrm{MHz}\right) \delta 7.2$ $7.40(\mathrm{~m}, 10 \mathrm{H}), 4.94(\mathrm{~d}, J=11.3 \mathrm{~Hz}, 1 \mathrm{H}), 4.90(\mathrm{~d}, J=11.1 \mathrm{~Hz}, 1 \mathrm{H}), 4.94(\mathrm{~d}, J=11.3$ Hz,1H), 4.89 (q, 2H, $J=11.3 \mathrm{~Hz}), 4.76(\mathrm{~d}, J=3.6 \mathrm{~Hz}, 1 \mathrm{H}), 4.68(\mathrm{~d}, J=11.1 \mathrm{~Hz}, 1 \mathrm{H})$, $3.83(\mathrm{dd}, J=2.5,11.7 \mathrm{~Hz}, 1 \mathrm{H}), 3.78(\mathrm{~d}, J=9.2 \mathrm{~Hz}, 1 \mathrm{H}), 3.74(\mathrm{dd}, J=4.0,11.9 \mathrm{~Hz}, 1 \mathrm{H})$, $3.7(\mathrm{~m}, 2 \mathrm{H}), 3.55(\mathrm{t}, J=9.3 \mathrm{~Hz}, 1 \mathrm{H}), 3.43(\mathrm{~s}, 3 \mathrm{H}), 1.99(\mathrm{br}, 1 \mathrm{H}) ;{ }^{13} \mathrm{C} \mathrm{NMR}\left(\mathrm{CDCl}_{3}, 100\right.$ MHz) $\delta 138.9,138.4,128.70,128.67,128.2,128.12,128.08,127.9,99.7,83.3,77.6,75.6$, 75.2, 73.2, 71.4, 61.9, 55.4; HRFAB Calcd for $\mathrm{C}_{21} \mathrm{H}_{26} \mathrm{O}_{6} \mathrm{Na}\left([\mathrm{M}+\mathrm{Na}]^{+}\right) \mathrm{m} / \mathrm{e} 397.1627$; measured m/e 397.1630.

\section{Phenyl 2,3-Di- $O$-benzyl-4,6- $O$-benzylidene-1-thio- $\beta$-D-galactopyranoside $(78) .{ }^{11}$}

Phenyl 2,3,4-Tri- $\boldsymbol{O}$-benzyl-1-thio- $\boldsymbol{\beta}$-D-galactopyranoside (79). ${ }^{1} \mathrm{H}$ NMR $\left(\mathrm{CDCl}_{3}, 400\right.$ MHz) $\delta 7.6(\mathrm{~m}, 2 \mathrm{H}), 7.2-7.4(\mathrm{~m}, 18 \mathrm{H}), 4.99(\mathrm{~d}, J=11.7 \mathrm{~Hz}, 1 \mathrm{H}), 4.84(\mathrm{~d}, J=10.2 \mathrm{~Hz}$, 1H), $4.775(\mathrm{~d}, J=10.2 \mathrm{~Hz}, 1 \mathrm{H}), 4.772(\mathrm{~d}, J=1.4 \mathrm{~Hz}, 1 \mathrm{H}), 4.66(\mathrm{~d}, J=9.6 \mathrm{~Hz}, 1 \mathrm{H}), 4.65$ (d, $J=11.7 \mathrm{~Hz}, 1 \mathrm{H}), 3.97(\mathrm{t}, J=9.4 \mathrm{~Hz}, 1 \mathrm{H}), 3.87(\mathrm{~d}, J=3.3 \mathrm{~Hz}, 1 \mathrm{H}), 3.85(\mathrm{dd}, J=7.0$, $11.1 \mathrm{~Hz}, 1 \mathrm{H}), 3.62$ (dd, $J=2.7,9.0 \mathrm{~Hz}, 1 \mathrm{H}), 3.54(\mathrm{dd}, J=5.1,11.2 \mathrm{~Hz}, 1 \mathrm{H}), 3.4(\mathrm{~m}, 1 \mathrm{H})$; ${ }^{13} \mathrm{C} \mathrm{NMR}\left(\mathrm{CDCl}_{3}, 100 \mathrm{MHz}\right) \delta$ 138.54, 138.47, 138.4, 134.2, 131.8, 129.1, 128.7, 128.6, $128.54,128.51,128.4,128.1,128.01,127.97,127.85,127.4,88.0,84.5,79.0,77.8,75.9$, 74.4, 73.6, 73.3, 62.5; HRFAB Calcd for $\mathrm{C}_{33} \mathrm{H}_{34} \mathrm{O}_{5} \mathrm{SNa}\left([\mathrm{M}+\mathrm{Na}]^{+}\right) \mathrm{m} / \mathrm{e}$ 565.2025; measured $\mathrm{m} / \mathrm{e} 565.2011$.

\section{Phenyl 2,3-Di- $O$-benzoyl-4,6- $O$-benzylidene-1-thio- $\beta$-D-galactopyranoside $(80) .{ }^{11}$}

Phenyl 2,3-Di- $\boldsymbol{O}$-benzoyl-4- $\boldsymbol{O}$-benzyl-1-thio- $\beta$-D-galactopyranoside (81). ${ }^{1} \mathrm{H}$ NMR $\left(\mathrm{CDCl}_{3}, 400 \mathrm{MHz}\right) \delta 7.9-8.0(\mathrm{~m}, 4 \mathrm{H}), 7.5(\mathrm{~m}, 4 \mathrm{H}), 7.3-7.4(\mathrm{~m}, 4 \mathrm{H}), 7.2-7.3(\mathrm{~m}, 8 \mathrm{H})$, $5.90(\mathrm{t}, J=10.0 \mathrm{~Hz}, 1 \mathrm{H}), 5.39(\mathrm{dd}, J=3.0,10.0 \mathrm{~Hz}, 1 \mathrm{H}), 4.96(\mathrm{~d}, J=9.9 \mathrm{~Hz}, 1 \mathrm{H}), 4.78$ 
(d, $J=11.6 \mathrm{~Hz}, 1 \mathrm{H}), 4.50(\mathrm{~d}, J=11.6 \mathrm{~Hz}, 1 \mathrm{H}), 4.19(\mathrm{~d}, J=2.5 \mathrm{~Hz}, 1 \mathrm{H}), 3.93(\mathrm{dd}, J=7.1$, $11.2 \mathrm{~Hz}, 1 \mathrm{H}), 3.81(\mathrm{dd}, J=5.0,7.0 \mathrm{~Hz}, 1 \mathrm{H}), 3.65(\mathrm{~m}, 1 \mathrm{H}), 1.75(\mathrm{br}, 1 \mathrm{H}) ;{ }^{13} \mathrm{C} \mathrm{NMR}$ $\left(\mathrm{CDCl}_{3}, 100 \mathrm{MHz}\right) \delta 166.2,165.5,137.6,133.7,133.4,132.8,132.6,130.1,130.0,129.8$, 129.2, 129.1, 128.72, 128.66, 128.6, 128.4, 128.2, 128.1, 86.8, 79.3, 76.3, 74.9, 73.9, 68.7, 62.2 .

6-(N-tert-Butyoxycarbonylamino)hexyl 2-acetamido-2-deoxy-3,4,6-tri- $O$-acetyl- $\beta$-Dglucopyranoside (82). ${ }^{1} \mathrm{H} \mathrm{NMR}\left(\mathrm{CDCl}_{3}, 400 \mathrm{MHz}\right) \delta 5.90(\mathrm{~d}, J=8.5 \mathrm{~Hz}, 1 \mathrm{H}), 5.35(\mathrm{t}, J$ $=9.8 \mathrm{~Hz}, 1 \mathrm{H}), 5.06(\mathrm{t}, J=9.7 \mathrm{~Hz}, 1 \mathrm{H}), 4.72(\mathrm{~d}, J=8.2 \mathrm{~Hz}, 1 \mathrm{H}), 4.65(\mathrm{br}, 1 \mathrm{H}), 4.26(\mathrm{dd}, J$ $=4.7,12.2 \mathrm{~Hz}, 1 \mathrm{H}), 4.14(\mathrm{dd}, J=2.3,12.2 \mathrm{~Hz}, 1 \mathrm{H}), 3.9(\mathrm{~m}, 1 \mathrm{H}), 3.7-3.8(\mathrm{~m}, 1 \mathrm{H}), 3.7$ (m, 1H), 3.5 (m, 1H), 3.1 (m, 2H), 2.09 (s, 3H), 2.04 (s, 3H), 2.03 (s, 3H), $1.96(\mathrm{~s}, 3 \mathrm{H})$, $1.6-1.7(\mathrm{~m}, 2 \mathrm{H}), 1.5(\mathrm{~m}, 2 \mathrm{H}), 1.45(\mathrm{~s}, 9 \mathrm{H}), 1.3-1.4(\mathrm{~m}, 2 \mathrm{H}) ;{ }^{13} \mathrm{C} \mathrm{NMR}\left(\mathrm{CDCl}_{3}, 100\right.$ MHz) $\delta 170.9$ (2 carbons), 170.5, 169.6, 155.3, 100.9, 72.5, 72.0, 69.8, 69.0, 62.4, 55.3, 40.7, 29.7, 28.9, 28.6 (3 carbons), 23.5 (2 carbons), 20.93 ( 2 carbons), 20.86, 20.82; HRFAB Calcd for $\mathrm{C}_{24} \mathrm{H}_{41} \mathrm{~N}_{2} \mathrm{O}_{11} \mathrm{Na}\left([\mathrm{M}+\mathrm{Na}]^{+}\right)$m/e 533.2710; measured m/e 533.2704.

6-Azidohexyl $2,3,4,6$-tetra- $\boldsymbol{O}$-acetyl- $\boldsymbol{\beta}$-D-galactopyranoside (83). ${ }^{1} \mathrm{H}$ NMR $\left(\mathrm{CDCl}_{3}\right.$, $400 \mathrm{MHz}) \delta 5.37(\mathrm{~d}, J=3.2 \mathrm{~Hz}, 1 \mathrm{H}), 5.16(\mathrm{dd}, J=8.0,10.5 \mathrm{~Hz}, 1 \mathrm{H}), 5.00(\mathrm{dd}, J=3.5$, $10.5 \mathrm{~Hz}, 1 \mathrm{H}), 4.44(\mathrm{~d}, J=7.9 \mathrm{~Hz}, 1 \mathrm{H}), 4.1-4.2(\mathrm{~m}, 2 \mathrm{H}), 3.9(\mathrm{~m}, 2 \mathrm{H}), 3.5(\mathrm{~m}, 1 \mathrm{H}), 3.2-$ $3.3(\mathrm{~m}, 2 \mathrm{H}), 2.13$ (s, 3H), 2.03 (s, 6H), $1.96(\mathrm{~s}, 3 \mathrm{H}), 1.6(\mathrm{~m}, 4 \mathrm{H}), 1.4(\mathrm{~m}, 4 \mathrm{H}) ;{ }^{13} \mathrm{C}$ NMR $\left(\mathrm{CDCl}_{3}, 100 \mathrm{MHz}\right) \delta 170.5,170.4,169.5$ (2 carbons), 101.6, 71.2, 70.8, 70.1, 69.2, 67.3, 51.6, 29.5, 29.0, 26.6, 25.6, 20.9, 20.85 (2 carbons), 20.77; HRFAB Calcd for $\mathrm{C}_{20} \mathrm{H}_{31} \mathrm{~N}_{3} \mathrm{O}_{10} \mathrm{Na}\left([\mathrm{M}+\mathrm{Na}]^{+}\right)$m/e 496.1907; measured m/e 496.1911.

Methyl 2-O-Benzoyl-4,6-O-benzylidene-3-O-(2,3,4,6-tetra- $O$-acetyl- $\alpha$-D-galactopyranosyl)- $\alpha$-D-glucopyranoside (84). ${ }^{10}{ }^{1} \mathrm{H} \mathrm{NMR}\left(\mathrm{CDCl}_{3}, 400 \mathrm{MHz}\right) \delta 7.4-8.1$ (m, 
$10 \mathrm{H}), 5.62(\mathrm{~s}, 1 \mathrm{H}), 5.33(\mathrm{dd}, J=0.7,3.3 \mathrm{~Hz}, 1 \mathrm{H}), 5.20(\mathrm{dd}, J=8.1,10.5 \mathrm{~Hz}, 1 \mathrm{H}), 5.13$ $(\mathrm{dd}, J=3.8,9.6 \mathrm{~Hz}, 1 \mathrm{H}), 5.05(\mathrm{~d}, J=3.8 \mathrm{~Hz}, 1 \mathrm{H}), 4.84(\mathrm{dd}, J=3.4,10.4 \mathrm{~Hz}, 1 \mathrm{H}), 4.76$ $(\mathrm{d}, J=8.0 \mathrm{~Hz}, 1 \mathrm{H}), 4.40(\mathrm{t}, J=9.4 \mathrm{~Hz}, 1 \mathrm{H}), 4.31(\mathrm{dd}, J=4.4,9.9 \mathrm{~Hz}, 1 \mathrm{H}), 4.13(\mathrm{q}, J=$ $7.6 \mathrm{~Hz}, 1 \mathrm{H}), 4.04(\mathrm{dd}, J=11.0,6.1 \mathrm{~Hz}, 1 \mathrm{H}), 3.9$ (m, 1H), $3.8-3.9$ (m, 2H), 3.75 (t, $J=$ $6.0 \mathrm{~Hz}, 1 \mathrm{H}), 3.40$ (s, 3H), 2.10 (s, 3H), 2.00 (s, 3H), 1.90 (s, 3H), 1.60 (s, 3H); ${ }^{13} \mathrm{C}$ NMR $\left(\mathrm{CDCl}_{3}, 100 \mathrm{MHz}\right) \delta 170.6,170.5,170.3,169.5,166.0,137.5,133.8,130.0$ (2C), 129.6, 129.2, 128.8 (2C), 128.3 (2C), 126.2 (2C), 101.7, 101.2, 97.8, 79.4, 77.4 (2C), 76.6, 73.8, 71.4, 70.7, 69.1, 69.0, 67.1, 62.8, 61.3, 55.6, 20.8 (2C), 20.7, 20.3.

\section{6-(N-tert-Butyoxycarbonylamino)hexyl $\quad 2,3,4-$ tri- $O$-acetyl- $\alpha$-L-rhamnopyranoside}

(85). ${ }^{1} \mathrm{H}$ NMR $\left(\mathrm{CDCl}_{3}, 400 \mathrm{MHz}\right) \delta 5.27(\mathrm{dd}, J=3.4,10.1 \mathrm{~Hz}, 1 \mathrm{H}), 5.20(\mathrm{dd}, J=1.7,3.4$ $\mathrm{Hz}, 1 \mathrm{H}), 5.04(\mathrm{t}, J=9.9 \mathrm{~Hz}, 1 \mathrm{H}), 4.70(\mathrm{~s}, 1 \mathrm{H}), 4.59(\mathrm{br}, 1 \mathrm{H}), 3.8-3.9(\mathrm{~m}, 1 \mathrm{H}), 3.6-3.7$ (m, 1H), $3.4(\mathrm{~m}, 1 \mathrm{H}), 3.1(\mathrm{~m}, 2 \mathrm{H}), 2.13(\mathrm{~s}, 3 \mathrm{H}), 2.04(\mathrm{~s}, 3 \mathrm{H}), 1.97(\mathrm{~s}, 3 \mathrm{H}), 1.6-1.7(\mathrm{~m}$, $2 \mathrm{H}), 1.5-1.6(\mathrm{~m}, 2 \mathrm{H}), 1.43(\mathrm{~s}, 9 \mathrm{H}), 1.3-1.4(\mathrm{~m}, 2 \mathrm{H}), 1.20(\mathrm{~d}, J=6.2 \mathrm{~Hz}, 3 \mathrm{H}) ;{ }^{13} \mathrm{C}$ NMR $\left(\mathrm{CDCl}_{3}, 100 \mathrm{MHz}\right) \delta 170.3,170.2,170.1,156.2,97.6,79.0,71.4,70.2,69.4,68.2$, 66.5, 41.0, 30.1, 29.2, 28.6 (3 carbons), 23.6, 21.1, 21.0, 20.9, 17.6; HRFAB Calcd for $\mathrm{C}_{22} \mathrm{H}_{38} \mathrm{NO}_{10}\left([\mathrm{MH}]^{+}\right) \mathrm{m} / \mathrm{e} 476.2496$; measured m/e 476.2477.

\section{Methyl 4,6- $O$-Benzylidene-2,3-bis- $O$-(2,3,4-tri- $O$-acetyl- $\alpha$-L-rhamnopyranosyl)- $\alpha$-D-} glucopyranoside (86). ${ }^{1} \mathrm{H}$ NMR $\left(\mathrm{CDCl}_{3}, 400 \mathrm{MHz}\right) \delta 7.5(\mathrm{~m}, 2 \mathrm{H}), 7.2-7.3(\mathrm{~m}, 3 \mathrm{H})$, $5.55(\mathrm{~s}, 1 \mathrm{H}), 5.2-5.3(\mathrm{~m}, 4 \mathrm{H}), 5.05(\mathrm{t}, J=9.9 \mathrm{~Hz}, 1 \mathrm{H}), 5.01(\mathrm{~d}, J=1.0 \mathrm{~Hz}, 1 \mathrm{H}), 4.95(\mathrm{t}$, $J=10.0 \mathrm{~Hz}, 1 \mathrm{H}), 4.89(\mathrm{~d}, J=3.5 \mathrm{~Hz}, 1 \mathrm{H}), 4.87(\mathrm{~d}, J=1.4 \mathrm{~Hz}, 1 \mathrm{H}), 4.29(\mathrm{dd}, J=4.4$, $10.0 \mathrm{~Hz}, 1 \mathrm{H}), 4.0-4.2(\mathrm{~m}, 3 \mathrm{H}), 3.84(\mathrm{dt}, J=4.6,10.1 \mathrm{~Hz}, 1 \mathrm{H}), 3.75(\mathrm{t}, J=10.1 \mathrm{~Hz}, 1 \mathrm{H})$, 3.65 (dd, $J=3.5,9.4 \mathrm{~Hz}, 1 \mathrm{H}), 3.55$ (t, $J=9.5 \mathrm{~Hz}, 1 \mathrm{H}), 3.40$ (s, 3H), 2.13 (s, 3H), 2.09 (s, 3H), 2.05 (s, 3H), 1.95 (s, 6H), 1.93 (s, 3H), 1.21 (d, $J=6.3 \mathrm{~Hz}, 3 \mathrm{H}), 0.73$ (d, $J=6.2 \mathrm{~Hz}$, $3 \mathrm{H}) ;{ }^{13} \mathrm{C} \mathrm{NMR}\left(\mathrm{CDCl}_{3}, 100 \mathrm{MHz}\right) \delta 170.3,170.2$ (2 carbons), 170.1, 169.9 (2 carbons), 
137.3, 129.2, 128.2, 126.6, 102.2, 100.3, 99.6, 98.1, 83.3, 79.9, 73.0, 71.5, 71.4, 70.1, 69,6, 69.3, 69.2, 68.8, 67.4, 66.2, 62.7, 55.2, 21.04, 21.00, 20.95 (2 carbons), 20.89, 20.8, 17.8, 16.8; HRFAB Calcd for $\mathrm{C}_{38} \mathrm{H}_{50} \mathrm{O}_{20} \mathrm{Na}\left([\mathrm{M}+\mathrm{Na}]^{+}\right) \mathrm{m} / \mathrm{e}$ 849.2793; measured m/e 849.2817 .

\section{Methyl 2,3-Di- $O$-benzyl-4,6-bis- $O$-(2,3,4-tri- $O$-acetyl- $\alpha$-L-rhamnopyranosyl)- $\alpha$-D-} glucopyranoside (87). ${ }^{1} \mathrm{H}$ NMR $\left(\mathrm{CDCl}_{3}, 400 \mathrm{MHz}\right) \delta 7.2$ - 7.4 (m, 10H), 5.28 (dd, $J=$ $3.5,10.0 \mathrm{~Hz}, 1 \mathrm{H}), 5.23(\mathrm{dd}, J=3.4,10.2 \mathrm{~Hz}, 1 \mathrm{H}), 5.15$ (dd, $J=1.6,3.4 \mathrm{~Hz}, 1 \mathrm{H}), 5.0-$ $5.1(\mathrm{~m}, 3 \mathrm{H}), 4.99(\mathrm{~d}, J=10.0 \mathrm{~Hz}, 1 \mathrm{H}), 4.89(\mathrm{~d}, J=1.2 \mathrm{~Hz}, 1 \mathrm{H}), 4.81(\mathrm{~d}, J=1.2 \mathrm{~Hz}, 1 \mathrm{H})$, $4.76(\mathrm{br}, 1 \mathrm{H}), 4.73(\mathrm{br}, 1 \mathrm{H}), 4.63(\mathrm{~d}, J=12.0 \mathrm{~Hz}, 1 \mathrm{H}), 4.59$ (d, $J=3.5 \mathrm{~Hz}, 1 \mathrm{H}), 4.0$ (m, 1H), $3.9(\mathrm{~m}, 3 \mathrm{H}), 3.77$ (dd, $J=2.9,9.8 \mathrm{~Hz}, 1 \mathrm{H}), 3.7$ (m, 2H), 3.61 (dd, $J=3.6,9.5 \mathrm{~Hz}$, 1H), 3.39 (s, 3H), 2.15 (s, 3H), 2.09 (s, 3H), 2.04 (s, 3H), 1.99 (s, 3H), 1.98 (s, 3H), 1.97 $(\mathrm{s}, 3 \mathrm{H}), 1.21(\mathrm{~d}, J=6.2 \mathrm{~Hz}, 3 \mathrm{H}), 0.82(\mathrm{~d}, J=6.2 \mathrm{~Hz}, 3 \mathrm{H}) ;{ }^{13} \mathrm{C} \mathrm{NMR}\left(\mathrm{CDCl}_{3}, 100\right.$ MHz) $\delta 170.7,170.3,170.2,170.0$ (2 carbons), 169.9, 138.9, 138.2, 128.6, 129.3, 128.1, $127.8,127.5,98.0,97.9,97.7,80.8,80.1,75.9,75.6,73.6,71.6,71.1,70.3,70.0,69.6$, 69.2, 69.2, 67.2, 66.5, 66.2, 55.4, 21.0, 20.98 (2 carbons), 20.91 (2 carbons), 20.9, 17.5, 17.2; MALDI Calcd for $\mathrm{C}_{45} \mathrm{H}_{58} \mathrm{O}_{20} \mathrm{Na}\left([\mathrm{M}+\mathrm{Na}]^{+}\right)$m/e 941.3414; measured m/e 941.3427.

6-Azidohexyl $2,3,4,6$-tetra- $\boldsymbol{O}$-acetyl- $\boldsymbol{\alpha}$-D-mannopyranoside (88). ${ }^{1} \mathrm{H}$ NMR $\left(\mathrm{CDCl}_{3}\right.$, $400 \mathrm{MHz}) \delta 5.33(\mathrm{dd}, J=3.4,10.0 \mathrm{~Hz}, 1 \mathrm{H}), 5.26(\mathrm{t}, J=9.9 \mathrm{~Hz}, 1 \mathrm{H}), 5.22(\mathrm{dd}, J=1.7$, $3.3 \mathrm{~Hz}, 1 \mathrm{H}), 4.79$ (d, $J=1.4 \mathrm{~Hz}, 1 \mathrm{H}), 4.27$ (dd, $J=5.3,12.2 \mathrm{~Hz}, 1 \mathrm{H}), 4.10$ (dd, $J=2.4$, $12.2 \mathrm{~Hz}, 1 \mathrm{H}), 3.97$ (ddd, $J=9.7,5.3,2.4 \mathrm{~Hz}, 1 \mathrm{H}), 3.7(\mathrm{~m}, 1 \mathrm{H}), 3.4-3.5(\mathrm{~m}, 1 \mathrm{H}), 3.27$ (t, $J=6.9 \mathrm{~Hz}, 2 \mathrm{H}), 2.14$ (s, 3H), 2.09 (s, 3H), 2.03 (s, 3H), 1.98 (s, 3H), $1.6(\mathrm{~m}, 4 \mathrm{H}), 1.4-$ $1.5(\mathrm{~m}, 4 \mathrm{H}) ;{ }^{13} \mathrm{C} \mathrm{NMR}\left(\mathrm{CDCl}_{3}, 100 \mathrm{MHz}\right) \delta 170.8,170.3,170.1,169.9,97.8,69.9,69.3$, $68.7,68.5,66.5,62.8,51.5,29.3,28.9,26.7,25.9,25.6,21.1,20.88,20.85$. HRFAB Calcd for $\mathrm{C}_{20} \mathrm{H}_{31} \mathrm{~N}_{3} \mathrm{O}_{10} \mathrm{Na}\left([\mathrm{M}+\mathrm{Na}]^{\dagger}\right)$ m/e 496.1907; measured m/e 496.1905. 


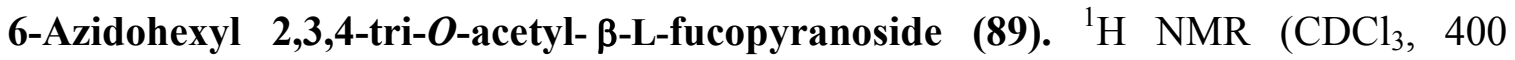
MHz) $\delta 5.24(\mathrm{~d}, J=2.6 \mathrm{~Hz}, 1 \mathrm{H}), 5.19(\mathrm{dd}, J=7.9,10.5 \mathrm{~Hz}, 1 \mathrm{H}), 5.02(\mathrm{dd}, J=3.3,10.4$ Hz, 1H), $4.43(\mathrm{~d}, J=7.9 \mathrm{~Hz}, 1 \mathrm{H}), 3.9(\mathrm{~m}, 1 \mathrm{H}), 3.80(\mathrm{dq}, J=0.8,6.5 \mathrm{~Hz}, 1 \mathrm{H}), 3.54(\mathrm{t}, J=$ $6.7 \mathrm{~Hz}, 1 \mathrm{H}), 3.4-3.5(\mathrm{~m}, 1 \mathrm{H}), 3.27$ (t, $J=6.9 \mathrm{~Hz}, 1 \mathrm{H}), 2.18$ (s, 3H), $2.06(\mathrm{~s}, 3 \mathrm{H}), 1.99$ (s, $3 \mathrm{H}), 1.6(\mathrm{~m}, 4 \mathrm{H}), 1.3-1.5(\mathrm{~m}, 4 \mathrm{H}), 1.24(\mathrm{~d}, J=6.5 \mathrm{~Hz}, 3 \mathrm{H}) ;{ }^{13} \mathrm{C} \mathrm{NMR}\left(\mathrm{CDCl}_{3}, 100\right.$ MHz) $\delta 170.9,170.4,169.6,101.4,71.6,70.6,79.9,69.3,51.6,32.7,29.5,29.0,26.6$, 25.6, 20.97, 20.89, 20.8, 16.3; HRFAB Calcd for $\mathrm{C}_{18} \mathrm{H}_{29} \mathrm{~N}_{3} \mathrm{O}_{8} \mathrm{Na}\left([\mathrm{M}+\mathrm{Na}]^{+}\right) \mathrm{m} / \mathrm{e}$ 438.1852; measured $\mathrm{m} / \mathrm{e} 438.1834$.

\section{Methyl 6-Azido-6-deoxy-2,3-di-O-benzyl- $\alpha$-D-glucopyranoside (90). ${ }^{8}$}

Methyl 6-Azido-6-deoxy-2,3-di- $O$-benzyl-4-O-(2,3,4-tri- $O$-acetyl- $\alpha$-L-rhamnopyranosyl)- $\alpha$-D-glucopyranoside $\quad$ (91). $\quad{ }^{1} \mathrm{H} \quad \mathrm{NMR} \quad\left(\mathrm{CDCl}_{3}, 400 \quad \mathrm{MHz}\right) \quad$ (major anomer) $\delta 7.3-7.4(\mathrm{~m}, 10 \mathrm{H}), 5.19(\mathrm{~d}, J=3.1 \mathrm{~Hz}, 1 \mathrm{H}), 5.15(\mathrm{dd}, J=8.4,10.4 \mathrm{~Hz}, 1 \mathrm{H})$, $4.97(\mathrm{dd}, J=8.0,9.0 \mathrm{~Hz}, 1 \mathrm{H})$, ), $4.96(\mathrm{~s}, 1 \mathrm{H}), 4.78(\mathrm{~d}, J=12.0 \mathrm{~Hz}, 1 \mathrm{H})$, ), $4.71(\mathrm{dd}, J=$ $13.8 \mathrm{~Hz}, 1 \mathrm{H})$, ), $4.67(\mathrm{~d}, J=12.8 \mathrm{~Hz}, 1 \mathrm{H}), 4.65(\mathrm{~d}, J=12.0 \mathrm{~Hz}, 1 \mathrm{H}), 4.62(\mathrm{~d}, J=3.6 \mathrm{~Hz}$, $1 \mathrm{H}), 3.88(\mathrm{~d}, J=8.8 \mathrm{~Hz}, 1 \mathrm{H}), 3.7-3.8(\mathrm{~m}, 1 \mathrm{H}), 3.70(\mathrm{t}, J=9.6 \mathrm{~Hz}, 1 \mathrm{H}), 3.6-3.7(\mathrm{~m}$, 1H), $3.60(\mathrm{dd}, J=13.1,2.4 \mathrm{~Hz}, 1 \mathrm{H}), 3.50(\mathrm{dd}, J=9.5,3.5 \mathrm{~Hz}, 1 \mathrm{H}), 3.42$ (s, 3H), 2.18 (s, 3H), $2.05(\mathrm{~s}, 3 \mathrm{H}), 2.00(\mathrm{~s}, 3 \mathrm{H}), 1.16(\mathrm{~d}, J=6.4 \mathrm{~Hz}, 3 \mathrm{H}) ;{ }^{13} \mathrm{C} \mathrm{NMR}\left(\mathrm{CDCl}_{3}, 100\right.$ MHz) $\delta 170.8,170.3,169.6,138.3,138.0,128.9$ (2C), 128.8 (2C), 128.4 (2C), 128.3 (4C), 100.6, 97.9, 81.6, 80.2, 76.0, 75.2, 73.4, 71.6, 70.5, 69.5, 69.4, 69.0, 55.6, 51.5, 21.1, 20.9, 20.8, 16.0; MALDI Calcd for $\mathrm{C}_{33} \mathrm{H}_{41} \mathrm{~N}_{3} \mathrm{O}_{12} \mathrm{Na}\left([\mathrm{M}+\mathrm{Na}]^{+}\right) \mathrm{m} / \mathrm{e}$ 694.2582; measured m/e 694.2585.

\section{2,3,4,6-Tetra- $O$-acetyl- $\alpha$-D-glucopyranosyl trichloroacetimidate (92). ${ }^{8}$}


6-(N-tert-Butyoxycarbonylamino)hexyl $\quad 2,3,4,6$-Tetra- $O$-acetyl- $\beta$-D-glucopyranoside (93). ${ }^{1} \mathrm{H} \mathrm{NMR}\left(\mathrm{CDCl}_{3}, 400 \mathrm{MHz}\right) \delta 5.65(\mathrm{~d}, J=8.34 \mathrm{~Hz}, 1 \mathrm{H}), 5.25(\mathrm{t}, J=9.49 \mathrm{~Hz}, 1 \mathrm{H})$, 5.13-5.07 (m, 3H), $4.30(\mathrm{dd}, J=4.36,12.52 \mathrm{~Hz}, 1 \mathrm{H}), 4.11(\mathrm{dd}, J=2.04,12.45 \mathrm{~Hz}, 1 \mathrm{H})$, 3.86-3.81 (m, 1H), $3.63(\mathrm{t}, J=6.33 \mathrm{~Hz}, 2 \mathrm{H}), 3.23-3.16(\mathrm{~m}, 2 \mathrm{H}), 2.07(\mathrm{~s}, 3 \mathrm{H}), 2.03(\mathrm{~s}, 3 \mathrm{H})$, $2.02(\mathrm{~s}, 3 \mathrm{H}), 2.00(\mathrm{~s}, 3 \mathrm{H}), 1.61-1.48(\mathrm{~m}, 4 \mathrm{H}), 1.43(\mathrm{~s}, 9 \mathrm{H}), 1.42-1.37(\mathrm{~m}, 2 \mathrm{H}) ;{ }^{13} \mathrm{C} \mathrm{NMR}$ $\left(\mathrm{CDCl}_{3}, 100 \mathrm{MHz}\right) \delta 170.8,170.2,169.7,169.6,153.9,93.0,73.0,72.6,70.5,68.1,62.3$, 61.7, 41.2, 32.3 (3 carbons), 29.5, 28.6, 23.1 ( 2 carbons), 20.9, 20.8, 20.7 (2 carbons).

\section{2,3,4,6-Tetra-O-benzoyl- $\alpha$-D-mannopyranosyl Bromide (94). ${ }^{17}$}

\section{Phenyl 4,6-O-benzylidene-2-O-(2,3,4,6-tetra- $O$-benzoyl- $\alpha$-D-mannopyranosyl)-1-} thio- $\beta$-D-galactopyranoside (95). ${ }^{1} \mathrm{H} \mathrm{NMR}\left(\mathrm{CDCl}_{3}, 300 \mathrm{MHz}\right) \delta 7.1-8.2(\mathrm{~m}, 35 \mathrm{H})$, $6.11(\mathrm{t}, J=10.5 \mathrm{~Hz}, 1 \mathrm{H}), 6.08(\mathrm{t}, J=2.7 \mathrm{~Hz}, 1 \mathrm{H}), 5.84(\mathrm{dd}, J=3.3,10.2 \mathrm{~Hz}, 1 \mathrm{H}), 5.6(\mathrm{~s}$, $1 \mathrm{H}), 5.50(\mathrm{dd}, J=3.3,9.6 \mathrm{~Hz}, 1 \mathrm{H}), 5.35(\mathrm{~d}, J=1.5 \mathrm{~Hz}, 1 \mathrm{H}), 4.91(\mathrm{~d}, J=9.6 \mathrm{~Hz}, 1 \mathrm{H})$, $4.59(\mathrm{~d}, J=3.6 \mathrm{~Hz}, 1 \mathrm{H}), 4.50(\mathrm{~d}, J=9.9 \mathrm{~Hz}, 1 \mathrm{H}), 4.49(\mathrm{t}, J=9.3 \mathrm{~Hz}, 1 \mathrm{H}), 4.1-4.2(\mathrm{~m}$, 2H), $4.03(\mathrm{dd}, J=2.7,12.9 \mathrm{~Hz}, 1 \mathrm{H}), 3.80(\mathrm{br}, 1 \mathrm{H}), 3.68(\mathrm{dd}, J=2.1 \mathrm{~Hz}, 12.6 \mathrm{~Hz}, 1 \mathrm{H})$; ${ }^{13} \mathrm{C} \mathrm{NMR}\left(\mathrm{CDCl}_{3}, 100 \mathrm{MHz}\right) \delta 166.5,166.1,165.8,165.4,165.3,137.9,134.1,133.6$, $133.5,133.4,133.1,132.6,132.4,130.9,130.4,130.0,129.9,129.7,129.6,129.5,129.4$, $129.3,129.2,129.0,128.8,128.63,128.56,128.53,128.46,128.1,126.5,101.0,99.9$, 87.2, 74.8, 74.5, 74.2, 70.6, 70.2, 70.0, 69.6, 69.4, 66.5, 62.1; HRESI Calcd for $\mathrm{C}_{60} \mathrm{H}_{50} \mathrm{O}_{15} \mathrm{~S}\left(\left[\mathrm{M}+\mathrm{NH}_{4}\right]^{+}\right) \mathrm{m} / \mathrm{e} 1060.3214$; measured m/e 1060.3204. 


\section{II. ${ }^{1} \mathrm{H}$ and ${ }^{13} \mathrm{C}$ Spectra for New Compounds}

\begin{tabular}{|c|c|c|}
\hline Entry & Compound Name & Page \\
\hline 1 & ${ }^{1}$ H NMR of Phenyl 3-O-Benzoyl-1-thio- $\beta$-D-galactopyranoside (18) & S26 \\
\hline 2 & ${ }^{13} \mathrm{C}$ NMR of Phenyl 3-O-Benzoyl-1-thio- $\beta$-D-galactopyranoside (18) & S27 \\
\hline 3 & ${ }^{1}$ H NMR of $2,3,4,6$-Tetra- $O$-acetyl- $\beta$-D-glucopyranosyl Azide (47) & $\mathrm{S} 28$ \\
\hline 4 & ${ }^{13} \mathrm{C}$ NMR of $2,3,4,6$-Tetra- $O$-acetyl- $\beta$-D-glucopyranosyl Azide (47) & S29 \\
\hline 5 & ${ }^{1}$ H NMR of $2,3,4,6$-Tetra- $O$-acetyl- $\beta$-D-galactopyranosyl Azide (49) & S30 \\
\hline 6 & ${ }^{13} \mathrm{C}$ NMR of $2,3,4,6$-Tetra- $O$-acetyl- $\beta$-D-galactopyranosyl Azide (49) & S31 \\
\hline 7 & ${ }^{1}$ H NMR of 2,3,4,6-Tetra-O-acetyl-D-mannopyranosyl Azide (51) & $\mathrm{S} 32$ \\
\hline 8 & ${ }^{13} \mathrm{C}$ NMR of 2,3,4,6-Tetra-O-acetyl-D-mannopyranosyl Azide (51) & S33 \\
\hline 9 & ${ }^{1}$ H NMR of 2,3,4-Tri-O-acetyl- $\alpha$-D-rhamnopyranosyl Azide (53) & S34 \\
\hline 10 & ${ }^{13} \mathrm{C}$ NMR of 2,3,4-Tri- $O$-acetyl- $\alpha$-D-rhamnopyranosyl Azide (53) & S35 \\
\hline 11 & $\begin{array}{l}{ }^{1} \text { H NMR of } 2,2^{\prime}, 3,3 ', 4,4^{\prime}-H e x a-O \text {-benzyl-6,6'-bis(4-n-hexyl-1H-1,2,3-triazol- } \\
\text { 1-yl)- } \alpha \text {-D-trehalose (56) }\end{array}$ & S36 \\
\hline 12 & $\begin{array}{l}{ }^{13} \mathrm{C} \text { NMR of } 2,2^{\prime}, 3,3 \text { ',4,4'-Hexa-O-benzyl-6,6'-bis(4-n-hexyl-1H-1,2,3-triazol- } \\
\text { 1-yl)- } \alpha \text {-D-trehalose (56) }\end{array}$ & S37 \\
\hline 13 & $\begin{array}{l}{ }^{1} \text { H NMR of } 2,2^{\prime}, 3,3,4,4^{\prime} \text {-Hexa- } O \text {-benzyl-6,6'-bis(4-n-octyl-1H-1,2,3-triazol- } \\
\text { 1-yl)- } \alpha \text {-D-trehalose }(58)\end{array}$ & $\mathrm{S} 38$ \\
\hline 14 & $\begin{array}{l}{ }^{13} \mathrm{C} \text { NMR of } 2,2^{\prime}, 3,3^{\prime}, 4,4^{\prime} \text {-Hexa- } O \text {-benzyl-6,6'-bis(4-n-octyl-1H-1,2,3-triazol- } \\
1-y l)-\alpha \text {-D-trehalose }(58)\end{array}$ & S39 \\
\hline 15 & $\begin{array}{l}{ }^{1} \mathrm{H} \\
\text { butyoxycarbonylmethyl-1H-1,2,3-triazol-1-yl)- } \alpha \text {-D-trehalose }(60)\end{array}$ & $\mathrm{S} 40$ \\
\hline 16 & $\begin{array}{l}{ }^{13} \mathrm{C} \\
\text { butyoxycarbonylmethyl-1H-1,2,3-triazol-1-yl)- } \alpha \text {-D-trehalose }(60)\end{array}$ & S41 \\
\hline 17 & $\begin{array}{l}{ }^{1} \mathrm{H} \text { NMR of 6-(4-tert-Butyoxycarbonylmethyl-1H-1,2,3-triazol-1-yl)hexyl } \\
2,3,4,6 \text {-tetra-O-acetyl- } \beta \text {-D-glucopyranoside (62) }\end{array}$ & S42 \\
\hline 18 & $\begin{array}{l}{ }^{13} \mathrm{C} \text { NMR of 6-(4-tert-Butyoxycarbonylmethyl-1H-1,2,3-triazol-1-yl)hexyl } \\
2,3,4,6 \text {-tetra-O-acetyl- } \beta \text {-D-glucopyranoside (62) }\end{array}$ & S43 \\
\hline 19 & ${ }^{1}$ H NMR of 6-Azidohexyl 2,3,4-tri- $O$-acetyl- $\alpha$-L-rhamnopyranoside (63) & S44 \\
\hline 20 & ${ }^{13} \mathrm{C}$ NMR of 6-Azidohexyl 2,3,4-tri-O-acetyl- $\alpha$-L-rhamnopyranoside (63) & $\mathrm{S} 45$ \\
\hline 21 & $\begin{array}{l}{ }^{1} \mathrm{H} \text { NMR of } 6-(4-n-H e x y l-1 H-1,2,3-\text { triazol-1-yl)hexyl } 2,3,4-\text { tri- } O \text {-acetyl- } \alpha-\mathrm{L}- \\
\text { rhamnopyranoside (64) }\end{array}$ & S46 \\
\hline 22 & $\begin{array}{l}{ }^{13} \mathrm{C} \text { NMR of } 6-(4-n \text {-Hexyl-1H-1,2,3-triazol-1-yl)hexyl } 2,3,4-\text { tri- } O \text {-acetyl- } \alpha-\mathrm{L}- \\
\text { rhamnopyranoside (64) }\end{array}$ & S47 \\
\hline 23 & $\begin{array}{l}{ }^{1} \mathrm{H} \text { NMR of6-(4-n-Octyl-1H-1,2,3-triazol-1-yl)hexyl } 2,3,4-\text { tri- } O \text {-acetyl- } \alpha \text {-L- } \\
\text { rhamnopyranoside (65) }\end{array}$ & $\mathrm{S} 48$ \\
\hline 24 & $\begin{array}{l}{ }^{13} \mathrm{C} \text { NMR of 6-(4-n-Octyl-1H-1,2,3-triazol-1-yl)hexyl 2,3,4-tri- } O \text {-acetyl- } \alpha \text {-L- } \\
\text { rhamnopyranoside (65) }\end{array}$ & S49 \\
\hline 25 & $\begin{array}{l}{ }^{1} \mathrm{H} \text { NMR of 6-(4-tert-Butyoxycarbonylmethyl-1H-1,2,3-triazol-1-yl)hexyl } \\
2,3,4 \text {-tri- } O \text {-acetyl- } \alpha \text {-L-rhamnopyranoside (66) }\end{array}$ & $\mathrm{S} 50$ \\
\hline 26 & $\begin{array}{l}{ }^{13} \mathrm{C} \text { NMR of 6-(4-tert-Butyoxycarbonylmethyl-1H-1,2,3-triazol-1-yl)hexyl } \\
2,3,4-\text {-tri-O-acetyl- } \alpha \text {-L-rhamnopyranoside }(66)\end{array}$ & S51 \\
\hline
\end{tabular}




\begin{tabular}{|c|c|c|}
\hline 27 & $\begin{array}{l}{ }^{1} \text { H NMR of Methyl 2,3,4-Tri- } O \text {-acetyl-6-deoxy-6-(4-n-hexyl-1H-1,2,3-triazol- } \\
\text { 1-yl)- } \alpha \text {-D-glucopyranoside (68) }\end{array}$ & S52 \\
\hline 28 & $\begin{array}{l}{ }^{13} \mathrm{C} \text { NMR of Methyl 2,3,4-Tri- } O \text {-acetyl-6-deoxy-6-(4-n-hexyl-1H-1,2,3- } \\
\text { triazol-1-yl)- } \alpha \text {-D-glucopyranoside (68) }\end{array}$ & S53 \\
\hline 29 & $\begin{array}{l}{ }^{1} \mathrm{H} \text { NMR of Methyl 2,3,4-Tri-O-acetyl-6-deoxy-6-(4-n-octyl-1H-1,2,3-triazol- } \\
\text { 1-yl)- } \alpha \text {-D-glucopyranoside (69) }\end{array}$ & S54 \\
\hline 30 & $\begin{array}{l}{ }_{1}^{13} \mathrm{C} \text { NMR of Methyl 2,3,4-Tri- } O \text {-acetyl-6-deoxy-6-(4-n-octyl-1H-1,2,3-triazol- } \\
\text { 1-yl)- } \alpha \text {-D-glucopyranoside (69) }\end{array}$ & S55 \\
\hline 31 & $\begin{array}{lllcc}{ }^{1} \mathrm{H} & \text { NMR } & \text { of } & \text { Methyl } & 2,3,4-T r i-O \text {-acetyl-6-deoxy-6-(4-n-tert- } \\
\text { butyoxycarbonylmethyl } & -1 \mathrm{H}-1,2,3-\text { triazol-1-yl)- } \alpha \text {-D-glucopyranoside }(70)\end{array}$ & S56 \\
\hline 32 & $\begin{array}{l}{ }^{13} \mathrm{C} \quad \text { NMR } \\
\text { butyoxycarbonylmethyl }\end{array}$ & S57 \\
\hline 33 & $\begin{array}{l}{ }^{1} \mathrm{H} \text { NMR of Phenyl 2,3-Di-O-benzoyl-4- } O \text {-benzyl-1-thio- } \beta \text {-D-glucopyranoside } \\
\text { (72) }\end{array}$ & S58,59 \\
\hline 34 & $\begin{array}{llcll}{ }^{13} \mathrm{C} & \text { NMR } & \text { of } & \text { Phenyl } & \text { 2,3-Di- } O \text {-benzoyl-4- } O \text {-benzyl-1-thio- } \beta \text {-D- } \\
\text { glucopyranoside }(72)\end{array}$ & S60 \\
\hline 35 & ${ }^{1}$ H NMR of Phenyl 4-O-Benzyl-1-thio- $\beta$-D-glucopyranoside (74) & S61 \\
\hline 36 & ${ }^{13} \mathrm{C}$ NMR of Phenyl 4-O-Benzyl-1-thio- $\beta$-D-glucopyranoside (74) & S62 \\
\hline 37 & ${ }^{1}$ H NMR of Methyl 3,4-Di-O-benzyl- $\alpha$-D-glucopyranoside (77) & S63 \\
\hline 38 & ${ }^{13} \mathrm{C}$ NMR of Methyl 3,4-Di-O-benzyl- $\alpha$-D-glucopyranoside (77) & S64 \\
\hline 39 & ${ }^{1}$ H NMR of Phenyl 2,3,4-Tri- $O$-benzyl-1-thio- $\beta$-D-galactopyranoside (79) & S65 \\
\hline 40 & ${ }^{13} \mathrm{C}$ NMR of Phenyl $2,3,4-T r i-O$-benzyl-1-thio- $\beta$-D-galactopyranoside (79) & S66 \\
\hline 41 & 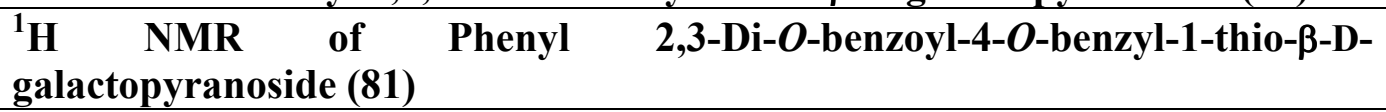 & S67 \\
\hline 42 & $\begin{array}{l}{ }^{13} \mathrm{C} \quad \text { NMR of } \\
\text { galactopyranoside (81) }\end{array}$ Phenyl 2,3-Di-O-benzoyl-4-O-benzyl-1-thio- $\beta$-D- & S68 \\
\hline 43 & $\begin{array}{l}\left.{ }^{1} \mathrm{H} \text { NMR of 6-( } N \text {-tert-Butyoxycarbonylamino }\right) \text { hexyl 2-acetamido-2-deoxy- } \\
3,4,6 \text {-tri- } O \text {-acetyl- } \beta \text {-D-glucopyranoside }(82)\end{array}$ & S69 \\
\hline 44 & $\begin{array}{l}{ }^{13} \mathrm{C} \text { NMR of } 6 \text { - }(N \text {-tert-Butyoxycarbonylamino }) \text { hexyl 2-acetamido-2-deoxy- } \\
3,4,6 \text {-tri- } O \text {-acetyl- } \beta \text {-D-glucopyranoside }(82)\end{array}$ & S70 \\
\hline 45 & ${ }^{1}$ H NMR of 6-Azidohexyl $2,3,4,6$-tetra- $O$-acetyl- $\beta$-D-galactopyranoside (83) & S71 \\
\hline 46 & ${ }^{13} \mathrm{C}$ NMR of 6-Azidohexyl 2,3,4,6-tetra- $O$-acetyl- $\beta$-D-galactopyranoside (83) & S72 \\
\hline 47 & $\begin{array}{l}{ }^{1} \mathrm{H} \text { NMR of } 6 \text { - }(\mathrm{N} \text {-tert-Butyoxycarbonylamino }) \text { hexyl } 2,3,4 \text {-tri- } O \text {-acetyl- } \alpha \text {-L- } \\
\text { rhamnopyranoside }(85)\end{array}$ & S73 \\
\hline 48 & $\begin{array}{l}{ }^{13} \mathrm{C} \text { NMR of } 6 \text { - }(N \text {-tert-Butyoxycarbonylamino)hexyl } 2,3,4 \text {-tri- } O \text {-acetyl- } \alpha \text {-L- } \\
\text { rhamnopyranoside }(85)\end{array}$ & S74 \\
\hline 49 & $\begin{array}{l}{ }^{1} \mathrm{H} \text { NMR of Methyl 4,6-O-Benzylidene-2,3-bis- } O \text {-(2,3,4-tri- } O \text {-acetyl- } \alpha \text {-L- } \\
\text { rhamnopyranosyl)- } \alpha \text {-D-glucopyranoside }(86)\end{array}$ & S75 \\
\hline 50 & $\begin{array}{l}{ }^{13} \mathrm{C} \text { NMR of Methyl 4,6-O-Benzylidene-2,3-bis- } O \text { - }(2,3,4-\text { tri- } O \text {-acetyl- } \alpha-\mathrm{L}- \\
\text { rhamnopyranosyl)- } \alpha \text {-D-glucopyranoside }(86)\end{array}$ & S76 \\
\hline 51 & $\begin{array}{l}{ }^{1} \mathrm{H} \text { NMR of Methyl 2,3-Di- } O \text {-benzyl-4,6-bis- } O \text {-(2,3,4-tri- } O \text {-acetyl- } \alpha \text {-L- } \\
\text { rhamnopyranosyl)- } \alpha \text {-D-glucopyranoside }(87)\end{array}$ & S77 \\
\hline 52 & ${ }^{13} \mathrm{C}$ NMR of Methyl 2,3-Di- $O$-benzyl-4,6-bis- $O$-(2,3,4-tri- $O$-acetyl- $\alpha$-L- & S78 \\
\hline
\end{tabular}




\begin{tabular}{|c|c|c|}
\hline & rhamnopyranosyl)- $\alpha$-D-glucopyranoside (87) & \\
\hline 53 & ${ }^{1} \mathrm{H}$ NMR of 6-Azidohexyl 2,3,4,6-tetra- $O$-acetyl- $\alpha$-D-mannopyranoside (88) & S79 \\
\hline 54 & ${ }^{13} \mathrm{C}$ NMR of 6-Azidohexyl 2,3,4,6-tetra- $O$-acetyl- $\alpha$-D-mannopyranoside (88) & S80 \\
\hline 55 & 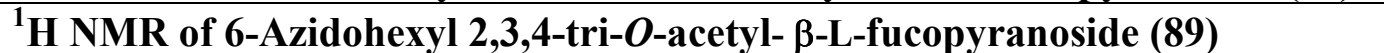 & S81 \\
\hline 56 & ${ }^{13}$ C NMR of 6-Azidohexyl 2,3,4-tri- $O$-acetyl- $\beta$-L-fucopyranoside (89) & S82 \\
\hline 57 & $\begin{array}{l}{ }^{1} H \text { NMR of Methyl 6-Azido-6-deoxy-2,3-di- } O \text {-benzyl-4- } O \text {-(2,3,4-tri- } O \text {-acetyl- } \\
\alpha \text {-L-rhamnopyranosyl)- } \alpha \text {-D-glucopyranoside (91) }\end{array}$ & S83 \\
\hline 58 & $\begin{array}{l}{ }^{13} \mathrm{C} \text { NMR of Methyl 6-Azido-6-deoxy-2,3-di- } O \text {-benzyl-4-O-(2,3,4-tri- } O \text { - } \\
\text { acetyl- } \alpha \text {-L-rhamnopyranosyl)- } \alpha \text {-D-glucopyranoside }(91)\end{array}$ & S84 \\
\hline 59 & $\begin{array}{l}{ }^{1} \text { H NMR of 6-( } N \text {-tert-Butyoxycarbonylamino)hexyl 2,3,4,6-Tetra- } O \text {-acetyl- } \beta- \\
\text { D-glucopyranoside (93). }\end{array}$ & S85 \\
\hline 60 & $\begin{array}{l}{ }^{13} \mathrm{C} \text { NMR of } 6 \text {-( }(\mathrm{N} \text {-tert-Butyoxycarbonylamino)hexyl } 2,3,4,6 \text {-Tetra- } O \text {-acetyl- } \\
\beta \text {-D-glucopyranoside }(93) \text {. }\end{array}$ & S86 \\
\hline 61 & $\begin{array}{l}{ }^{1} \mathrm{H} \text { NMR of Phenyl 4,6-O-benzylidene-2- } O \text { - }(2,3,4,6 \text {-tetra- } O \text {-benzoyl- } \alpha \text {-D- } \\
\text { mannopyranosyl)-1-thio- } \beta \text {-D-galactopyranoside (95). }\end{array}$ & S87 \\
\hline 62 & $\begin{array}{l}{ }^{13} \mathrm{C} \text { NMR of Phenyl 4,6-O-benzylidene-2- } O \text { - }(2,3,4,6 \text {-tetra- } O \text {-benzoyl- } \alpha \text {-D- } \\
\text { mannopyranosyl)-1-thio- } \beta \text {-D-galactopyranoside (95). }\end{array}$ & S88 \\
\hline
\end{tabular}



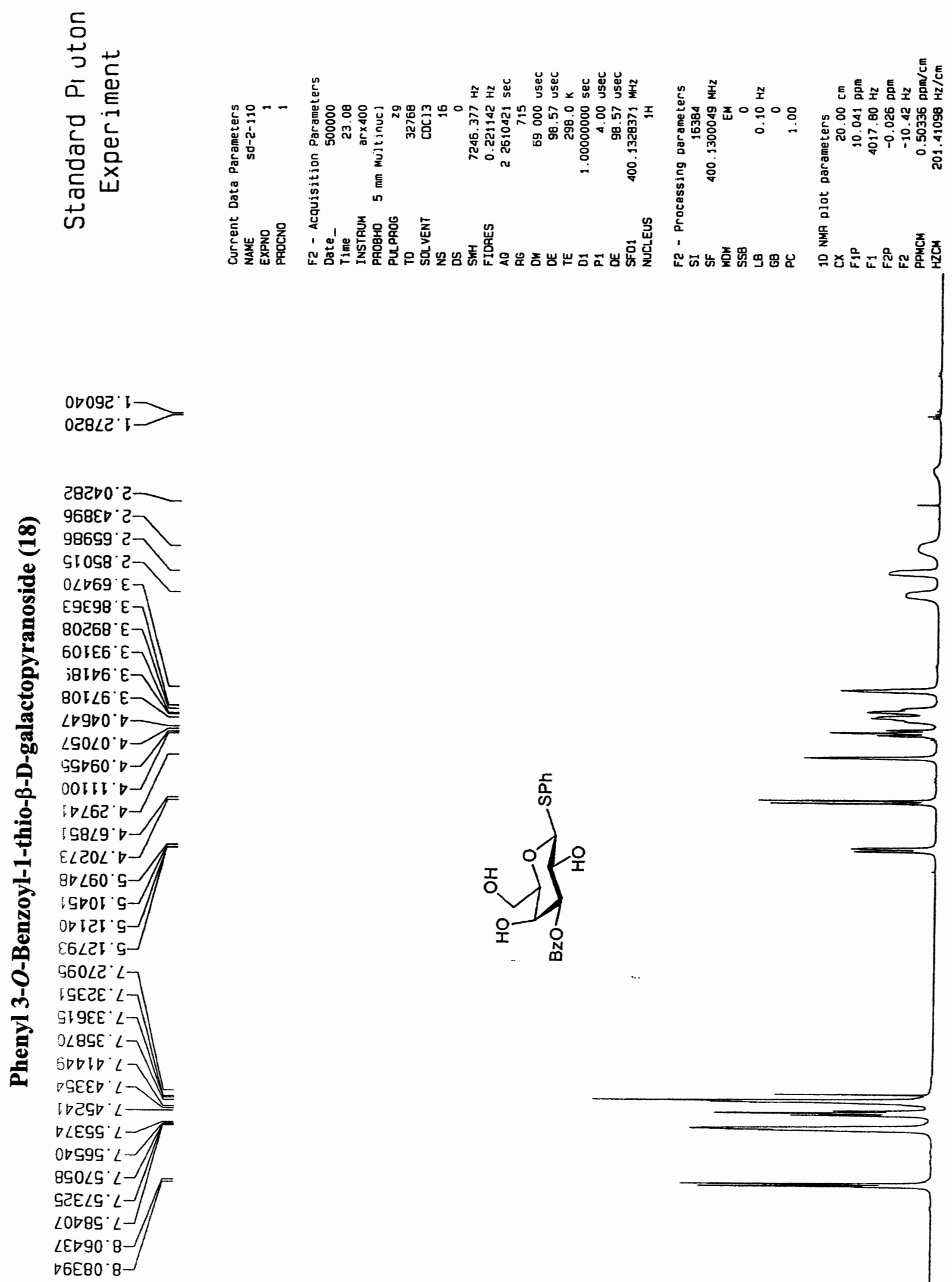

$0 D 095 \cdot 1>$
$028 \angle 2 \cdot 1-$

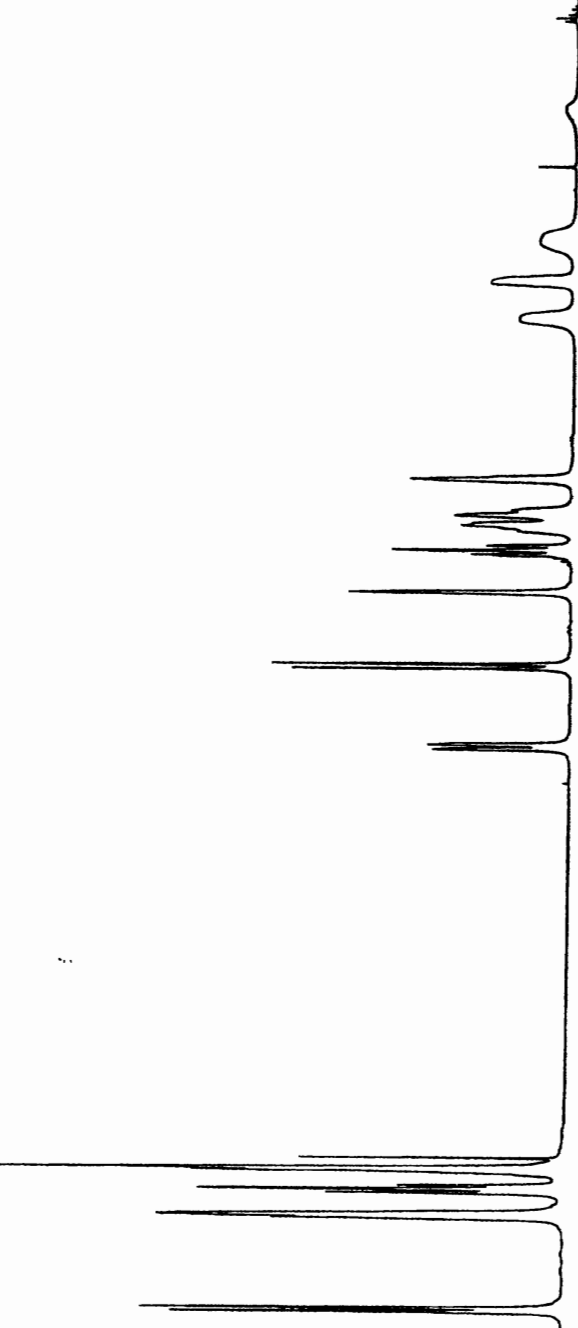

SटE $\angle G^{\circ}$

$\angle \varepsilon \triangleright 90^{\circ}$

$\triangle 6 E 80^{\circ} 8$

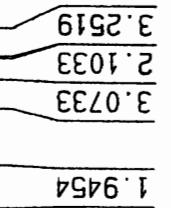




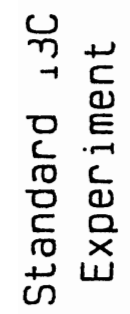

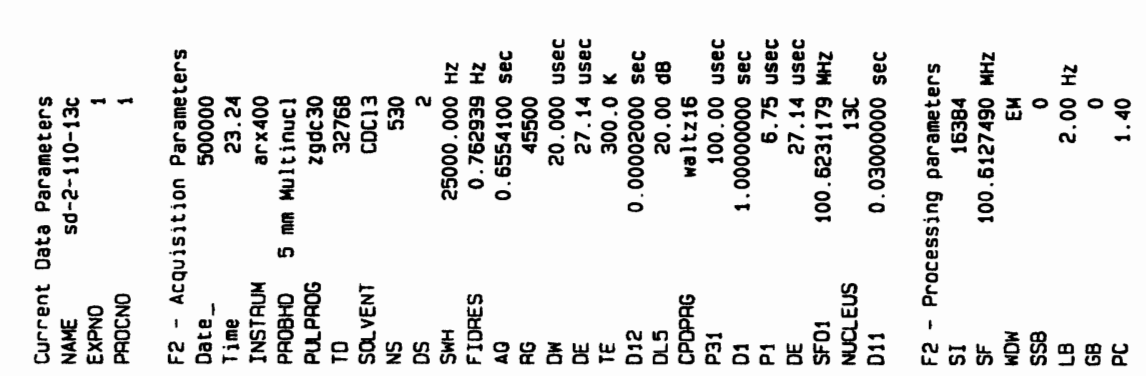

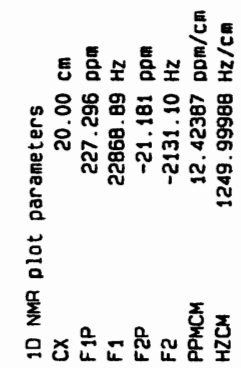
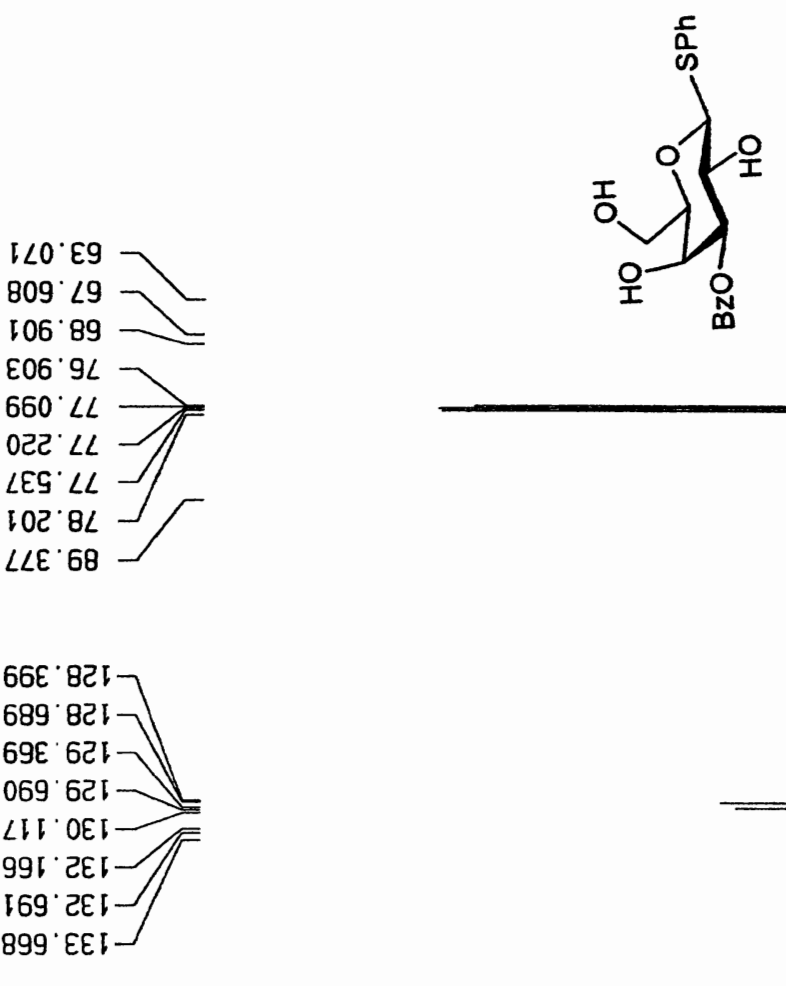

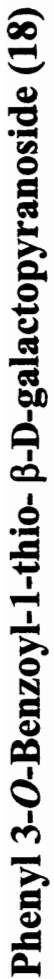

$\angle 00^{\circ} 991$

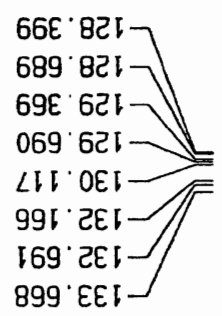




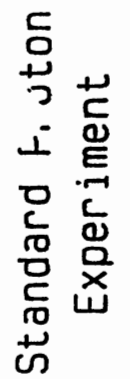
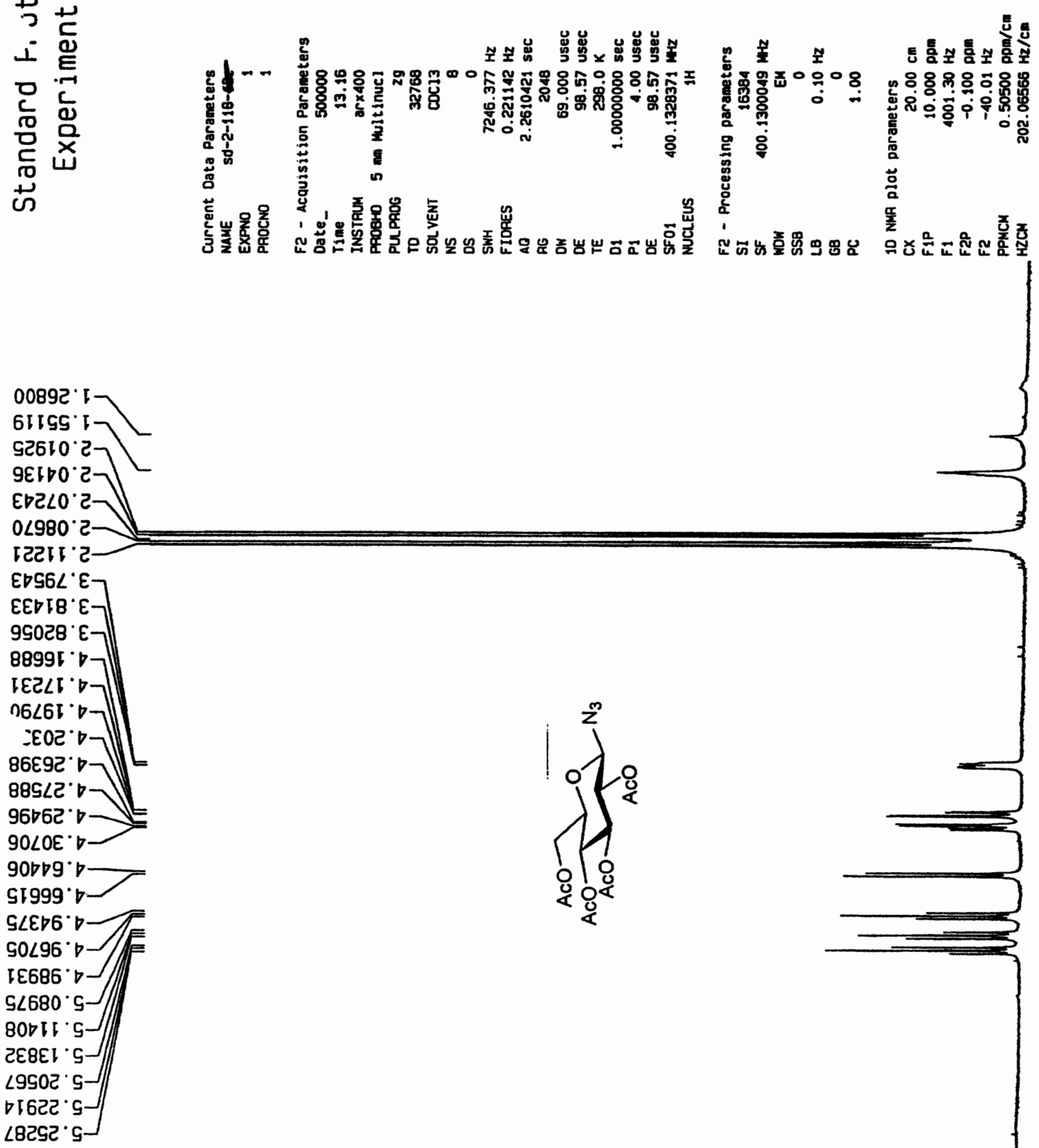

69692 .

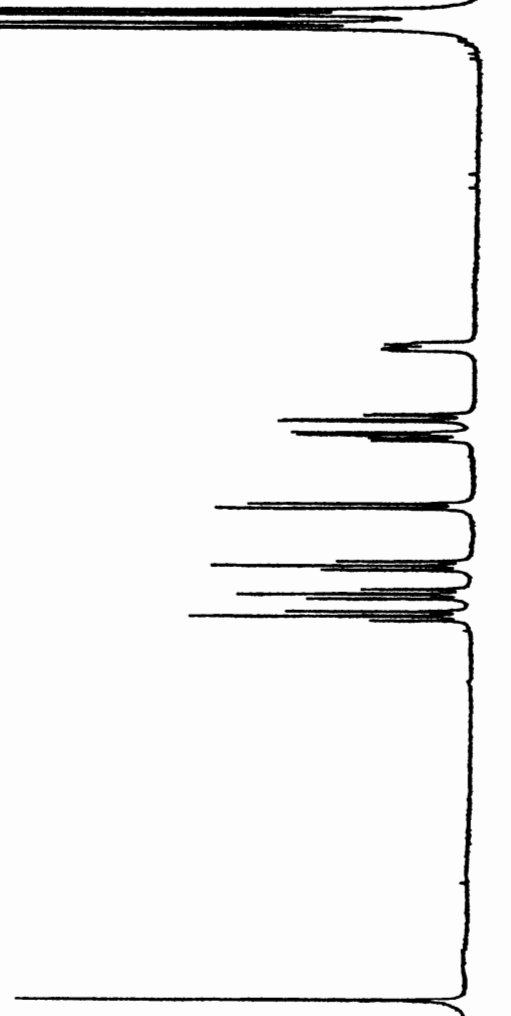

EOt $E I$

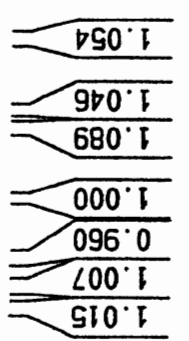



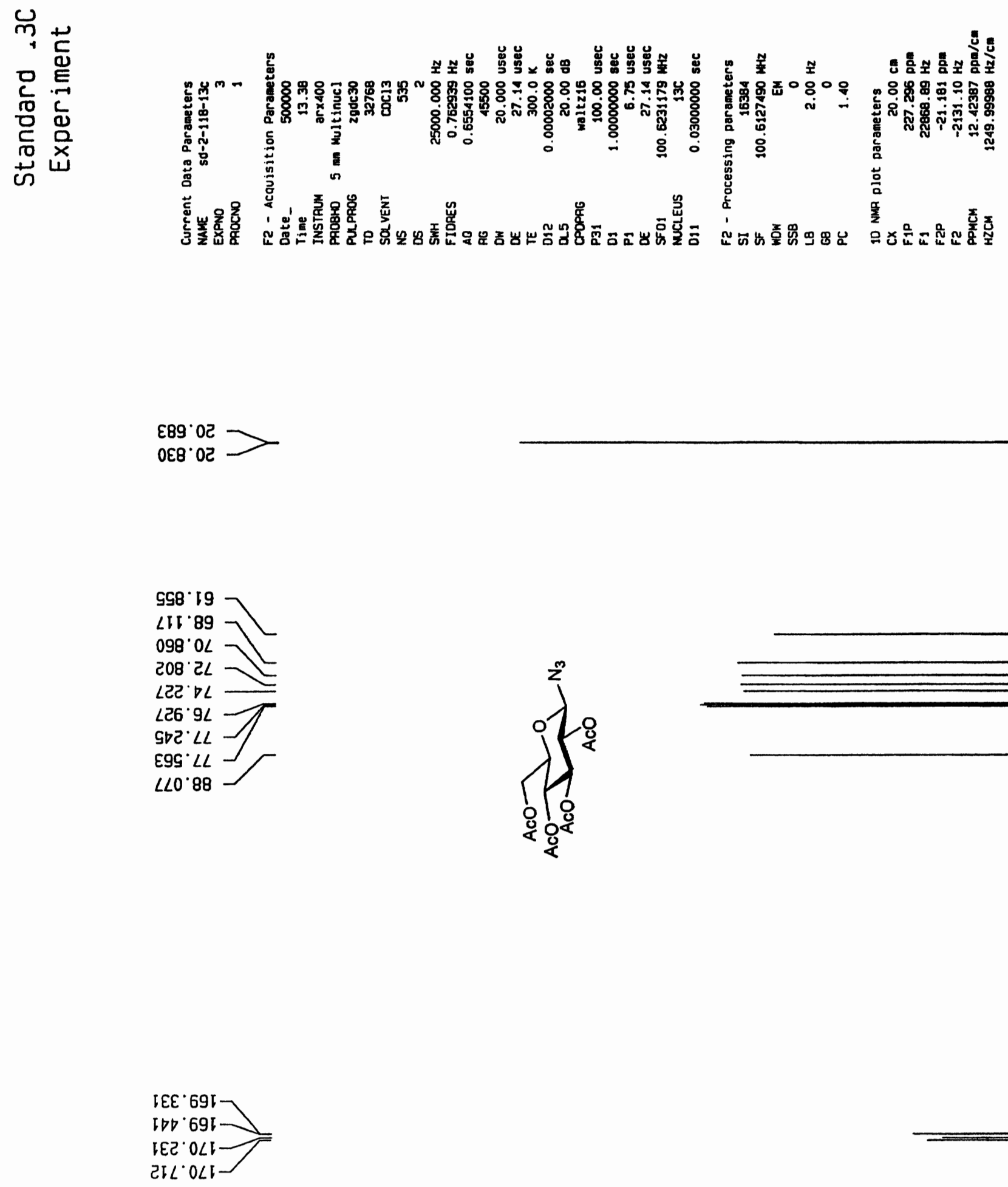

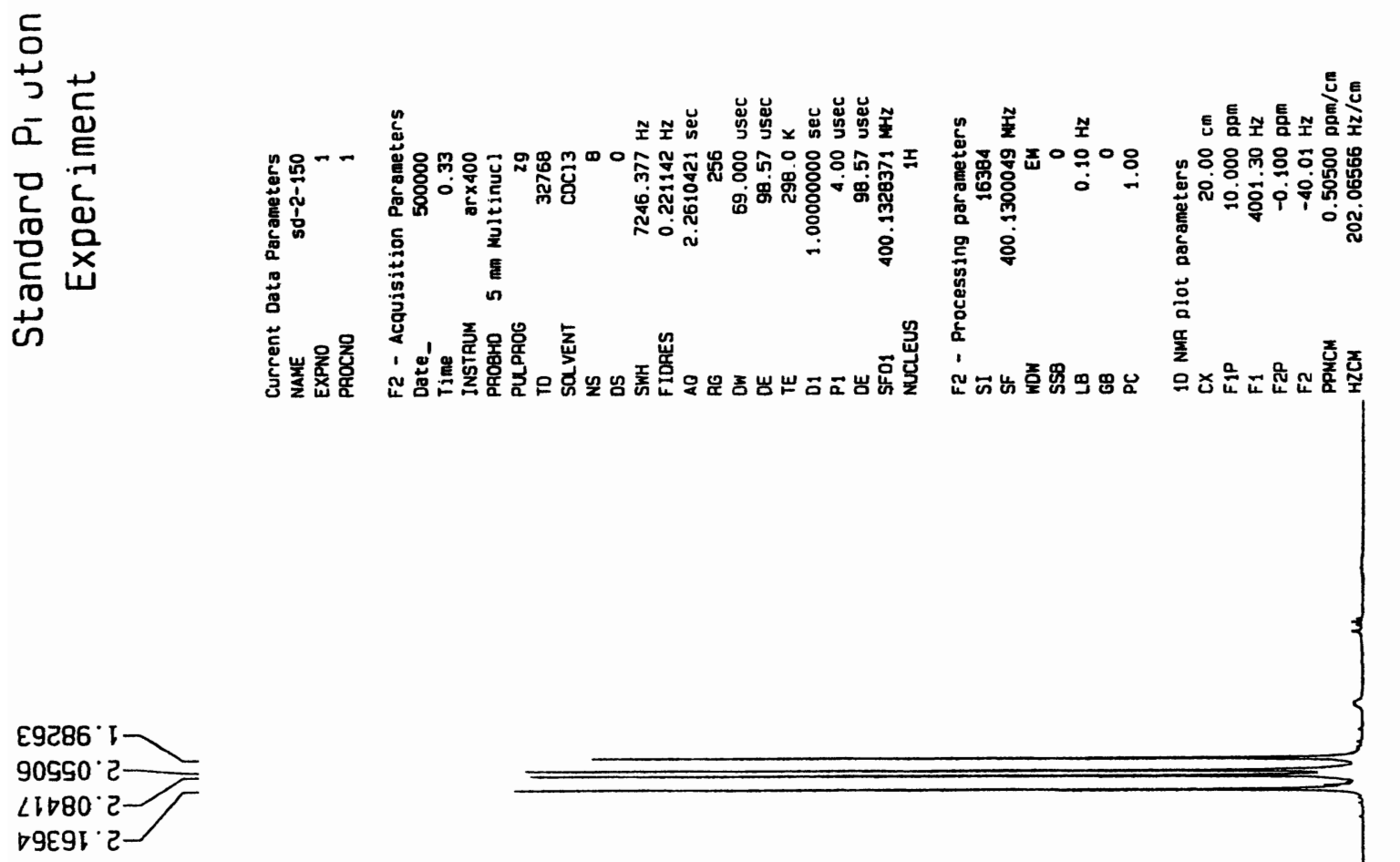

6ร566.

$82866^{\circ} \varepsilon-$

$98210^{\circ} \circ$

$96820^{\circ}$

i90E0.

$89091^{\circ} \cdot$

$8 G \angle \angle T \cdot \square]$

ESS85. $\square$

OD $\angle 09^{\circ}$

टL8IO'S-

$6\left[\angle 20^{\circ} 9\right.$

stob0's-

L6290 ${ }^{\circ}$

$\checkmark$ $D E$ ' $\mathrm{S}$

6ट95I. $\mathrm{s}$

E865I' $\mathrm{s}$

ट०टा० 9

0902D 9

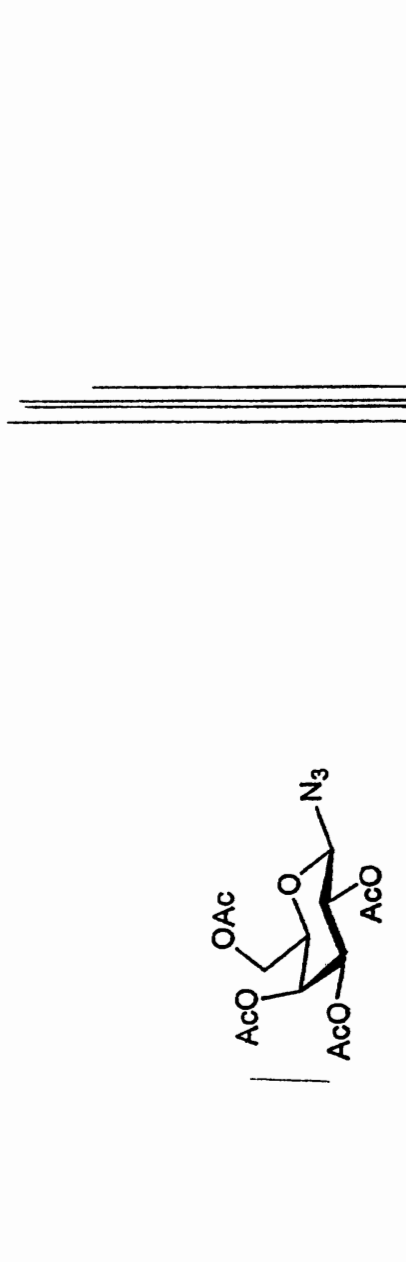

$\lceil\angle O L 2$

政 

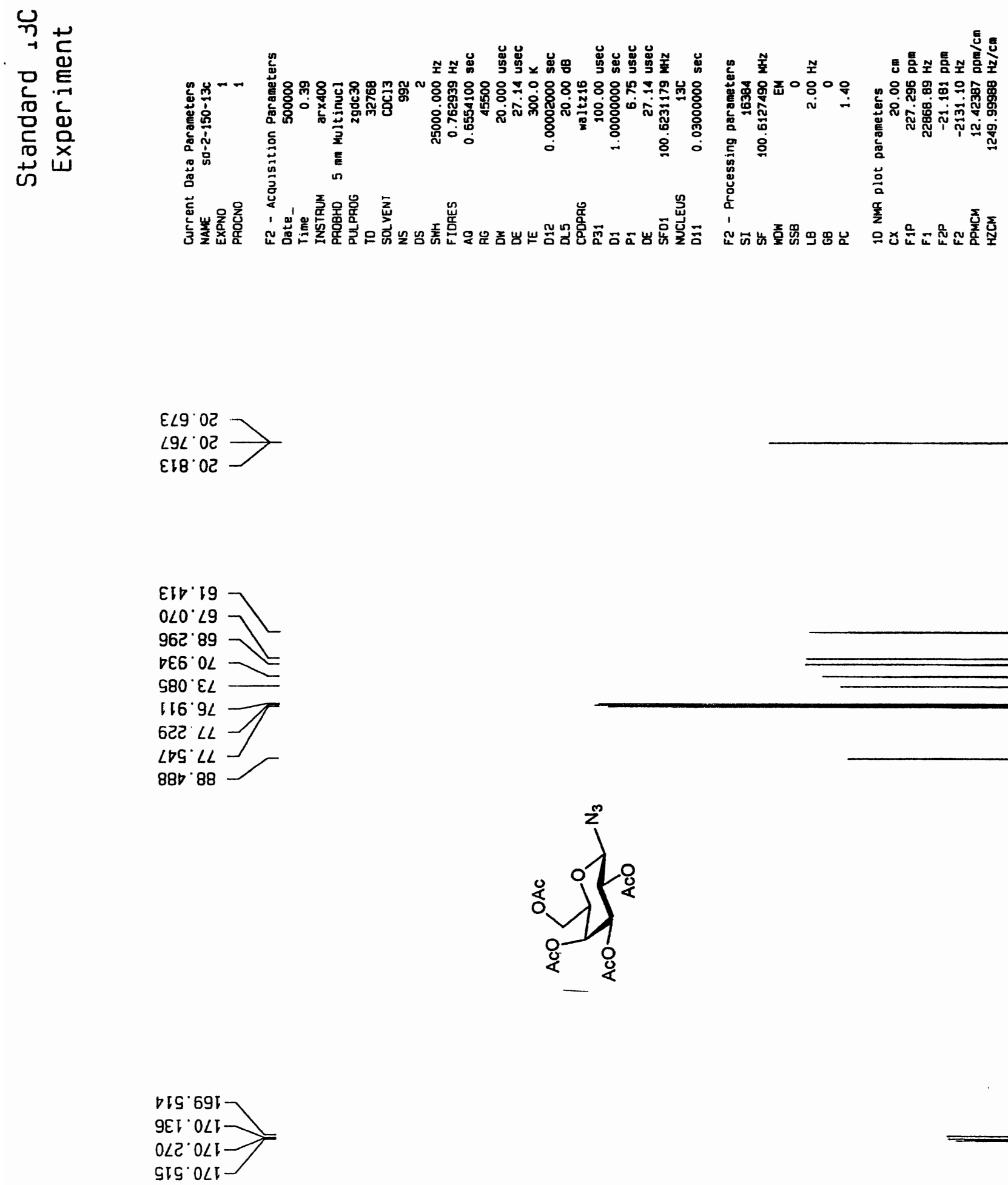

GIG $0 \angle 1$ 

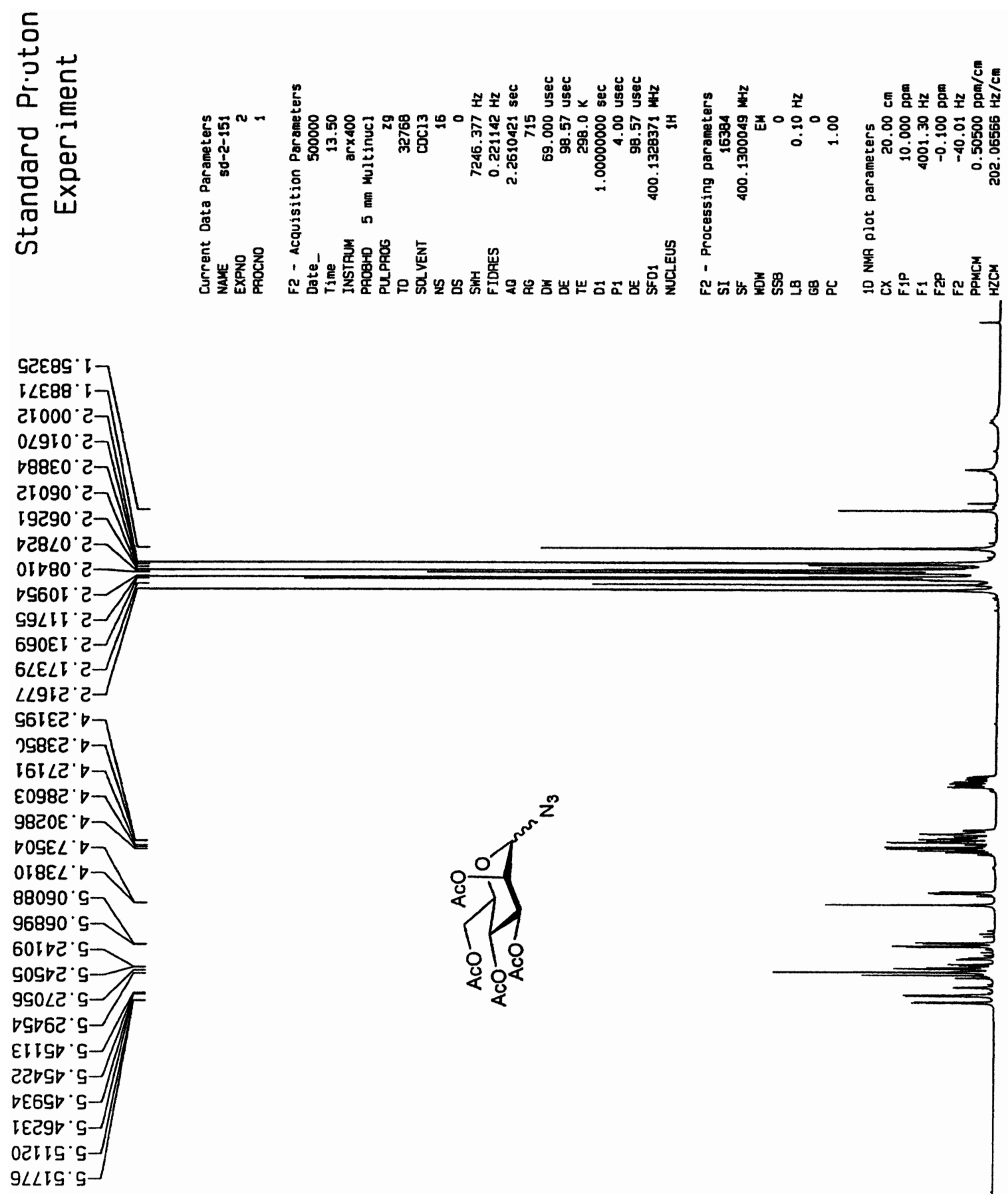

EटILZ $L$ 

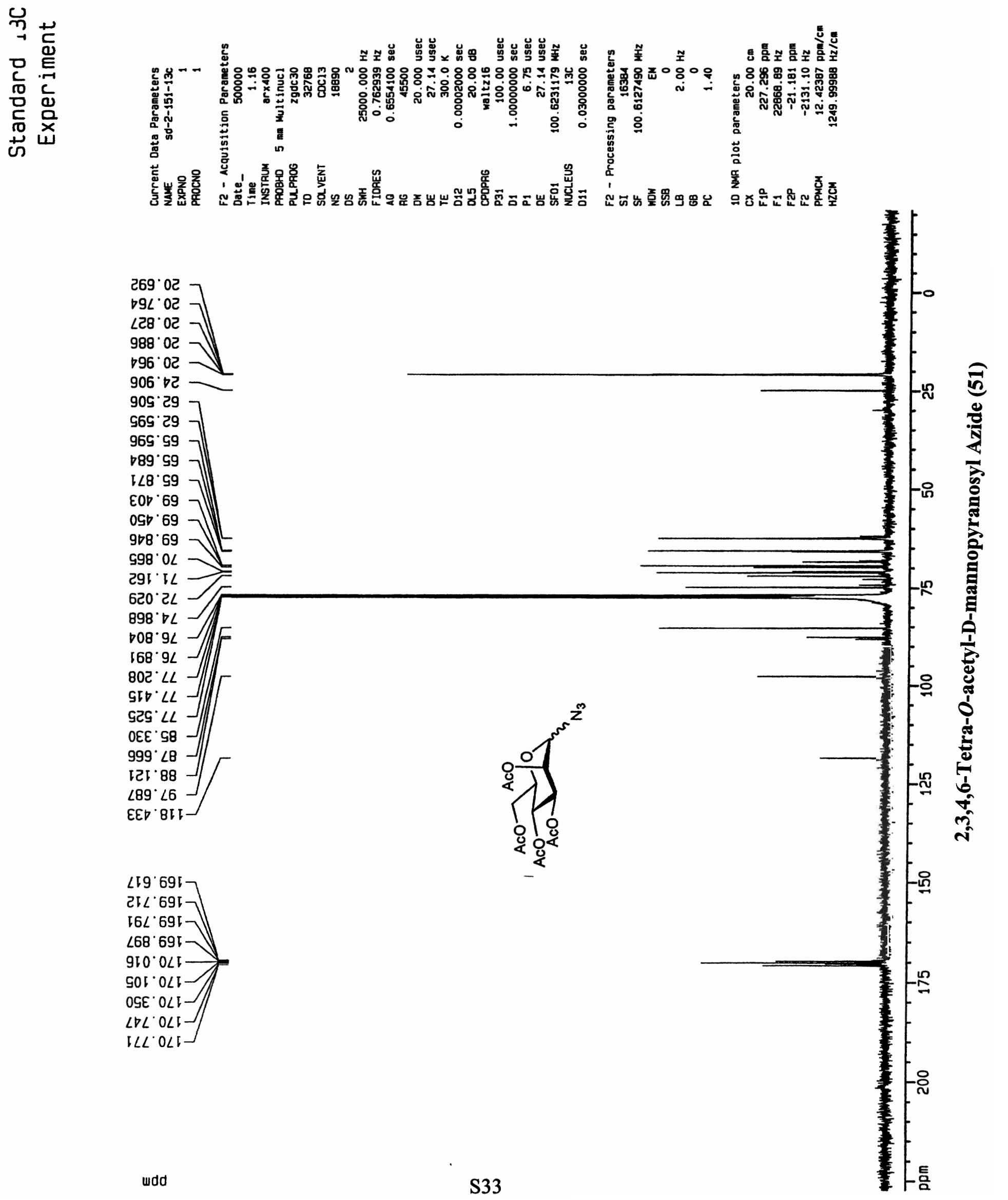

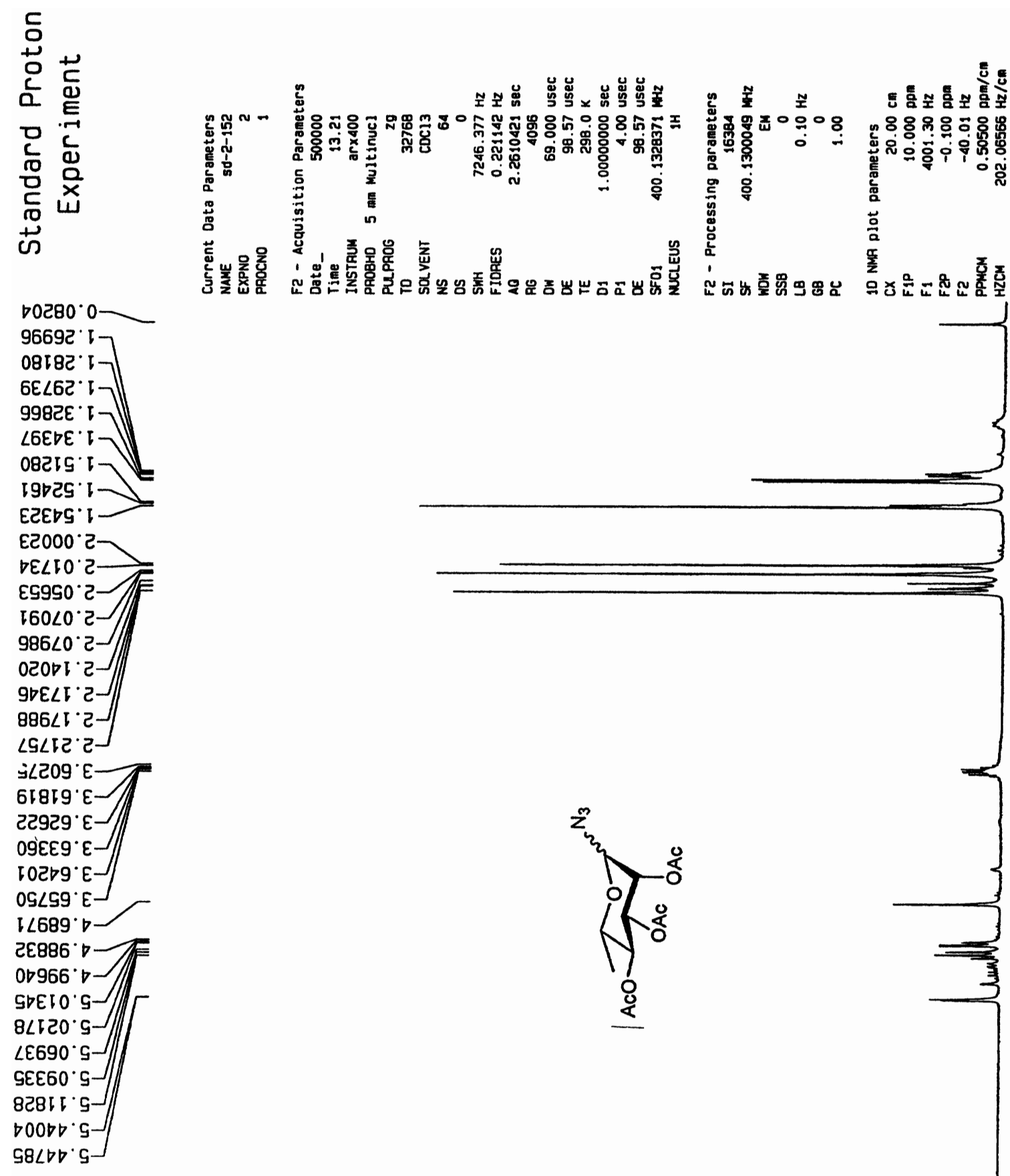

OEटLટ $L-$ 


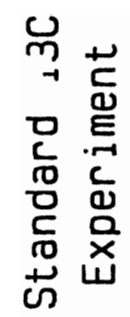

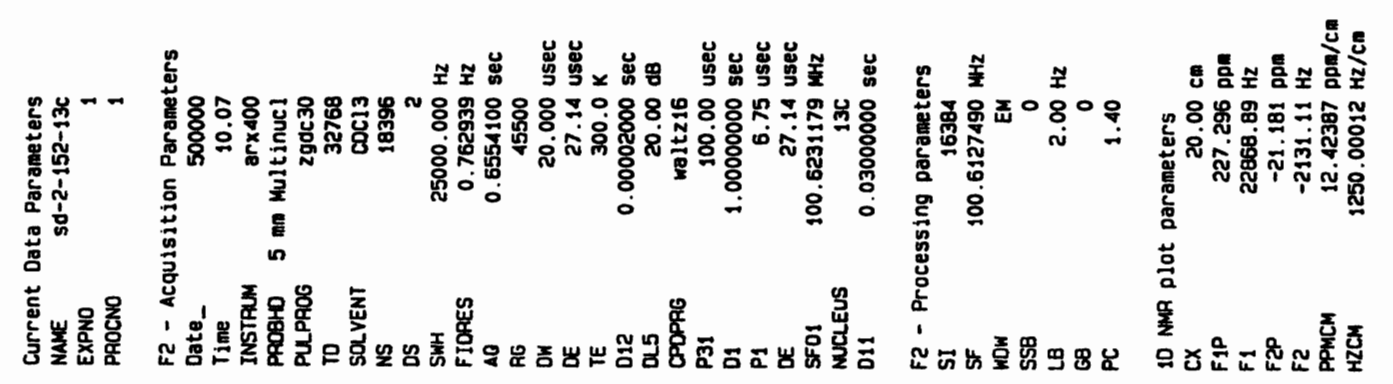

B9. $\angle I=$
$0 V L \cdot 02=$
$0 E 6^{\circ} 02=$

018.69-

$\angle 6 I^{\circ} 0 \angle>$

$8 \angle L^{\circ} \varepsilon \angle \perp$

$088^{\circ} 9 L$

$\angle 6 I^{\circ} \angle L$

00r. $\angle L$

sis $\angle L$

$9 \angle \mathrm{T} \cdot \mathrm{S} 8$

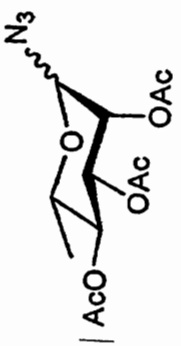

$\angle 22 \cdot 0 \angle 1-$ 


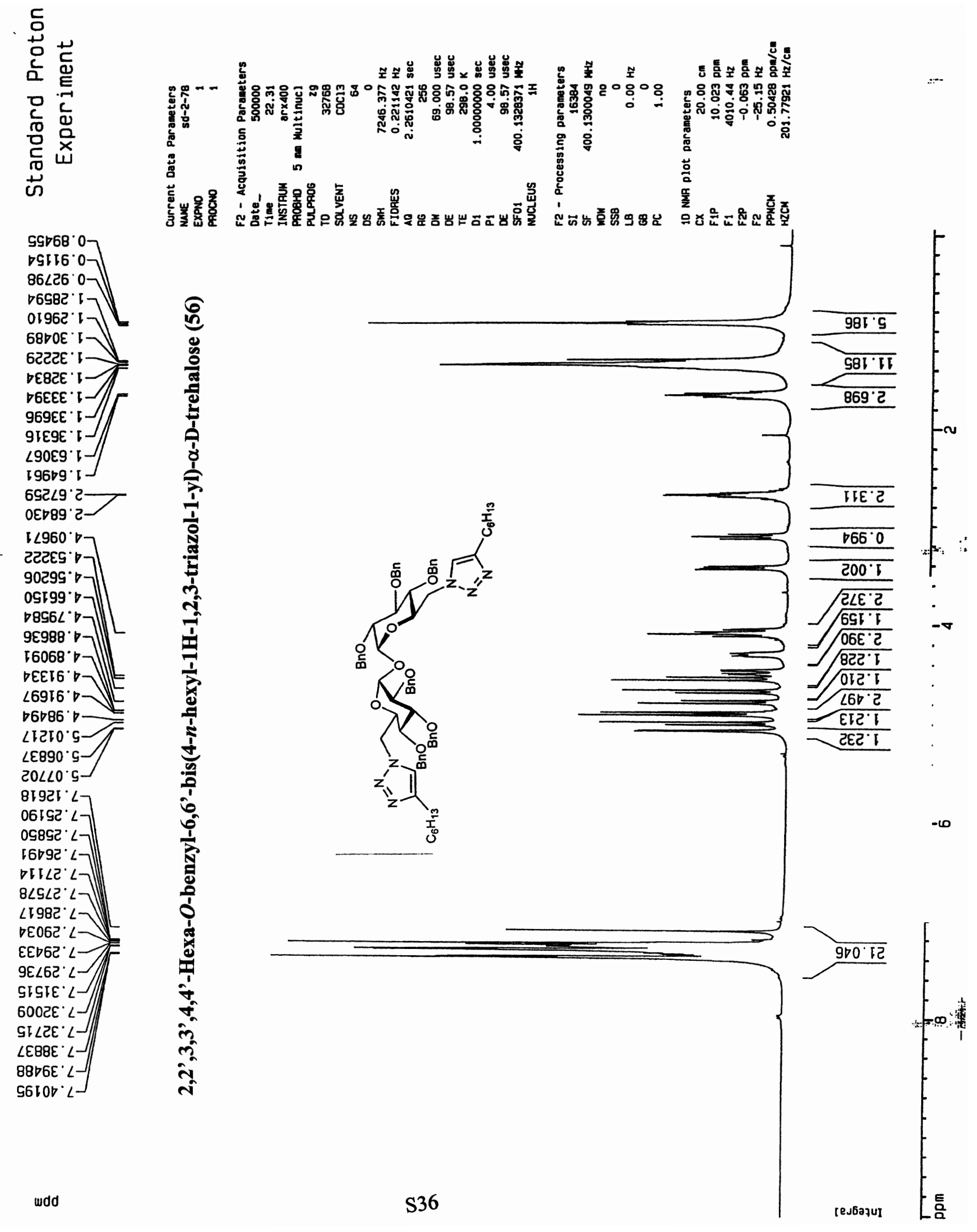




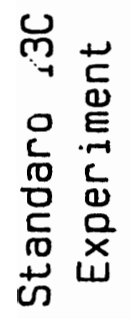
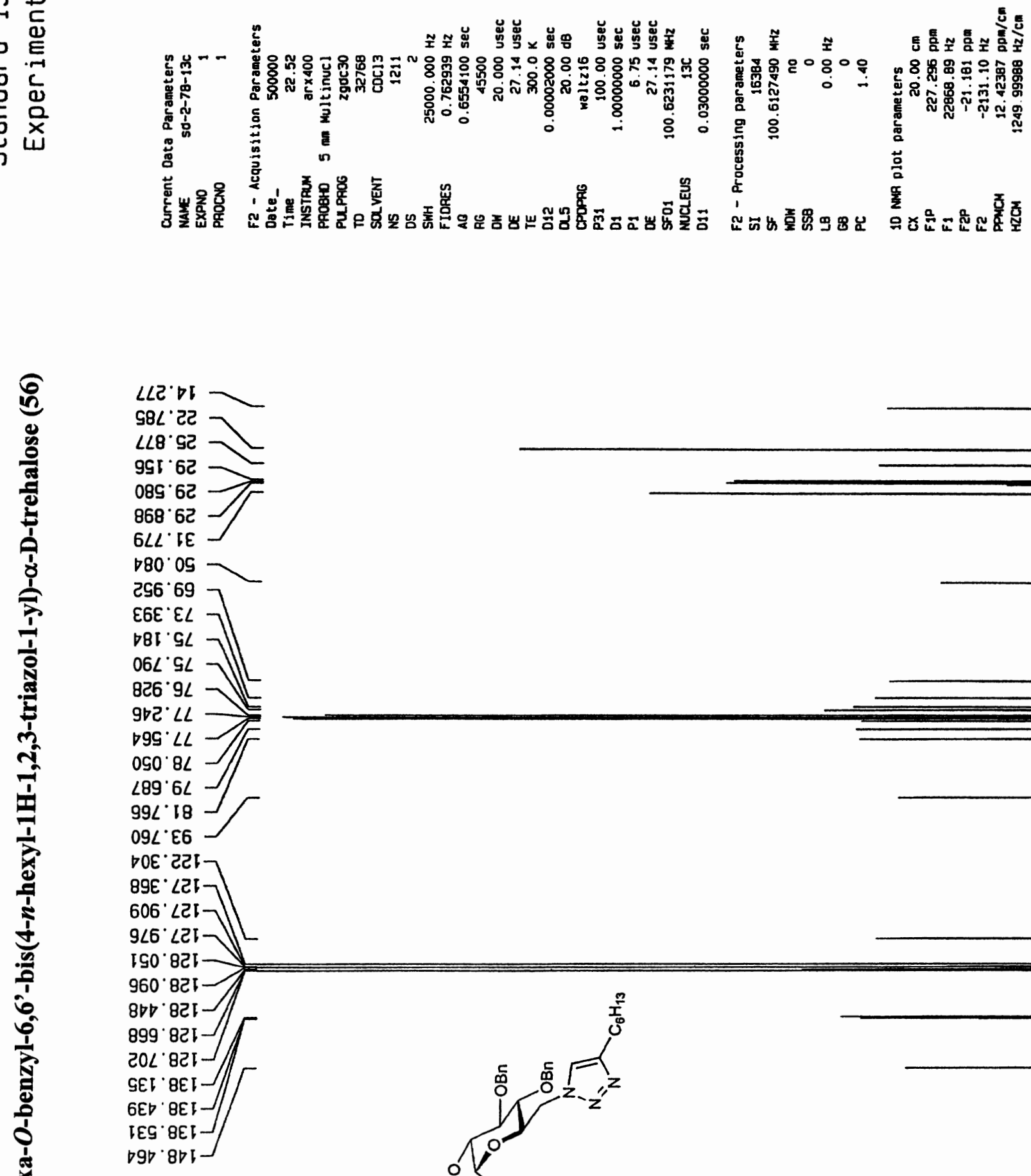

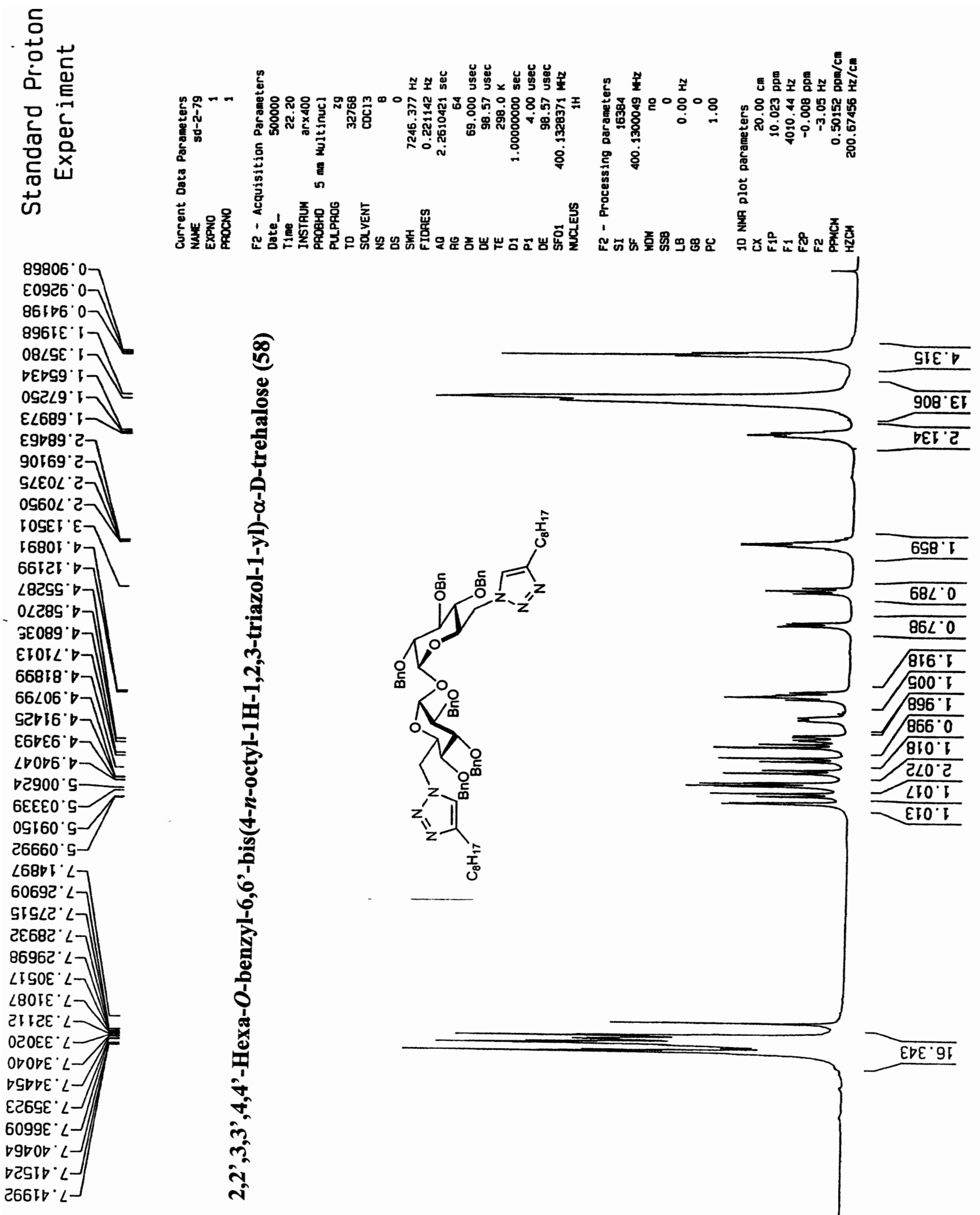


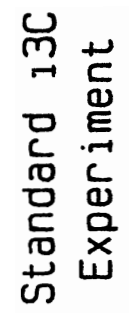

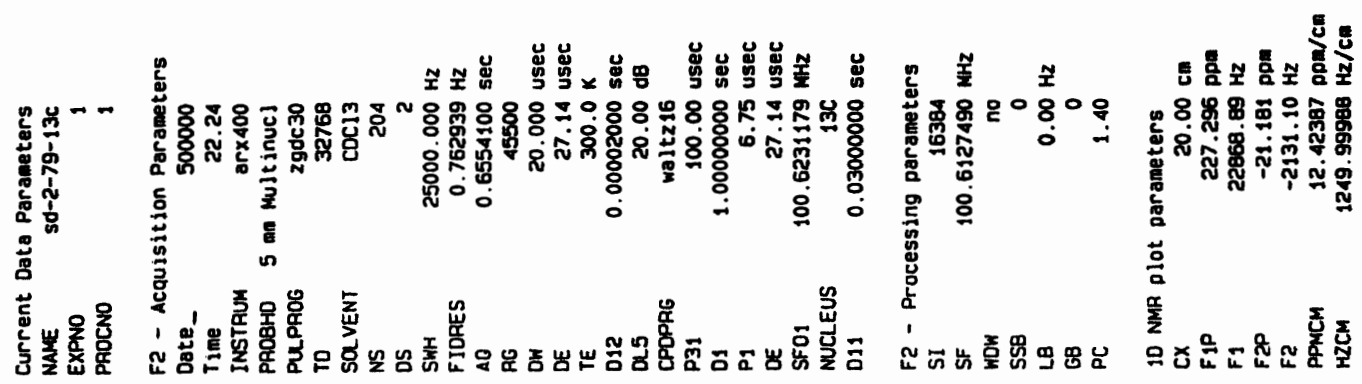

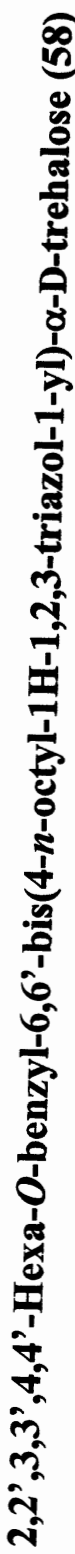

OtE' $\nabla$

ह68. टट

OI6 9

$\varepsilon \angle b^{\circ} 6 C^{\circ}$

$679^{\circ} 62$

$6 \angle 9^{\circ} 62$

$599^{\circ} 62$

$960^{\circ} \mathrm{्E}$

III.OS $\longrightarrow$

$166^{\circ} 69$

$\angle O D^{\circ} E L-$

OOZ'GL

$908^{\circ} \mathrm{SL}$

$\triangle 00^{\circ} \mathrm{LL}$

टटE $\angle L$

Ob9. $\angle L$

SOL'6L

$18 L \cdot 18$

टEL'E6

6IE टटा

$\left.\begin{array}{l}000^{\circ} \angle C I \\ 626^{\circ}[\mathrm{LCI}\end{array}\right]$

$066^{\circ} \angle 己 \vdash$

$880^{\circ} 851$

OाT $8 \mathrm{~B}$

9LO Bटा -

6LL'8ZI 821

$89 I^{\circ} 8 E \div-1$

$\left\ulcorner\angle V^{\circ} 8 E 5-\right.$

GLS. $8 E I-$

$96 b^{\circ} 8 \mathrm{~L}-$ 

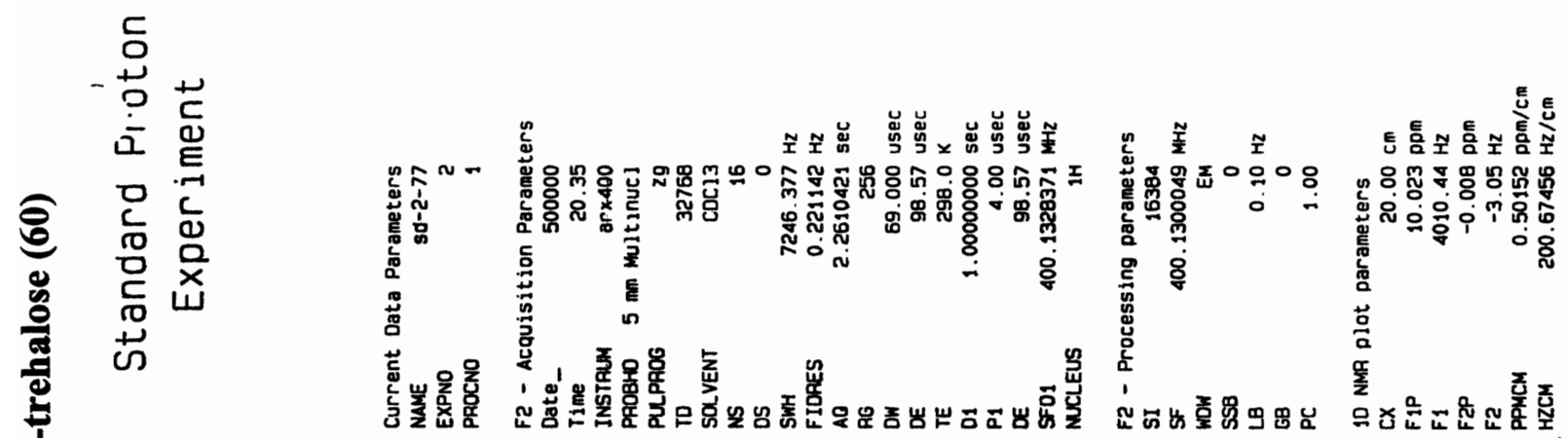

$666 \angle 2$

SDCOE' 1

[ $\angle S O E \cdot 1]$

$69900^{\circ}[]$

टLC90. 1

टा860 1

टटटIS

$68090^{\circ} \varepsilon$

$9 \angle E 80^{\circ} \varepsilon$

โBE โ० $\varepsilon]$

816टD

88LED $E-$

$\angle \nabla \angle 90$

Sट $\angle E$

8009E.

$ट \angle L \angle E^{\circ} \nabla$

3L6ES

$\angle \nabla 699^{\circ} \circ 7$

$0 \angle 899^{\circ}$

SICSL

$898 \angle L$

$\angle O I \angle 8^{\circ}$

$618 \angle 8^{\circ}$

$\angle S \angle 60^{\circ} \circ$

9DS06. $\square$

$\angle 2986^{\circ} \circ$

8DELO's-

$00080^{\circ} \mathrm{s}$

0 टG०ट $\angle$

$8 \angle 6 \nabla C$

$\left.\angle O \angle G C^{\circ} \angle\right]$

$6809 C^{\circ} \angle$

$\angle E O L C^{\circ} \angle$

$\angle 29 \angle C^{\circ}$

ट $\angle 180^{\circ}$

$\angle \nabla S B C^{\circ} \angle$

$\triangle \square 26 C^{\circ} \angle$

E9OIE $L$

$\angle \angle 9 V E^{\circ} \angle$

IEEटE $L-$

$\nabla \angle S B E^{\circ} \angle-$

$9896 E^{\circ} L-$

र955० $L$

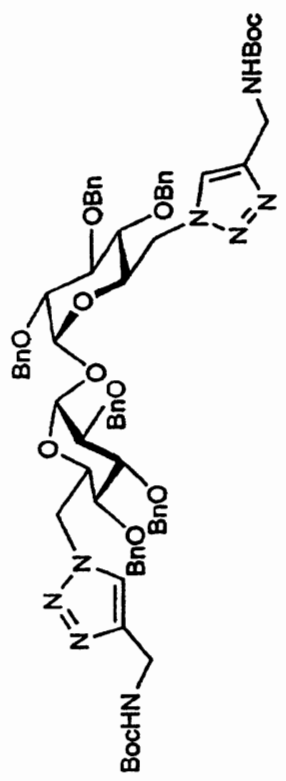




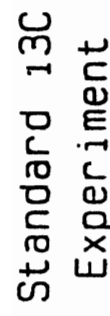

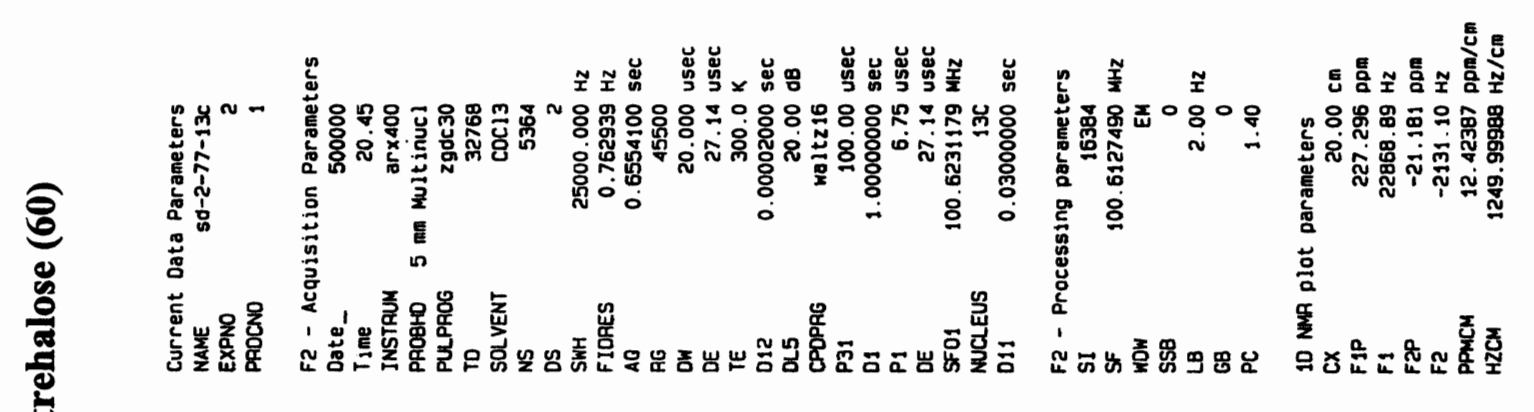

5ट9. 85

6SE. 98

$620^{\circ} 05$

SEL' 69

$\left.\begin{array}{l}\text { टट॰ } \varepsilon L \\ \nabla ट 己 \cdot G L\end{array}\right]$

$\nabla B L ' S L$

$\angle 26 \cdot 9 \angle$

SVD' $L L$

$\angle \forall V^{\circ} \angle L$

E9S $L L$

GLL' $6 L$

टह8. $6 L$

$89 L^{\circ}$ ' 18

टGE $\nabla 6$

$999 \cdot \varepsilon 己$

89E $\angle 2$

$6 \angle 9^{\circ} \angle C I$

II6 $\angle$ '

ᄃट0 8टा

Gटा ' 8टा-

$\varepsilon \angle V^{\circ} 821-$

[99. 821-

E89.8टा -

$\angle \forall L B 25]$

QL $8.85-\int$

IBE' $B E[-$

IOS. $8 E[-$

IIE SDI-

S00.991

लू

कू

กั 


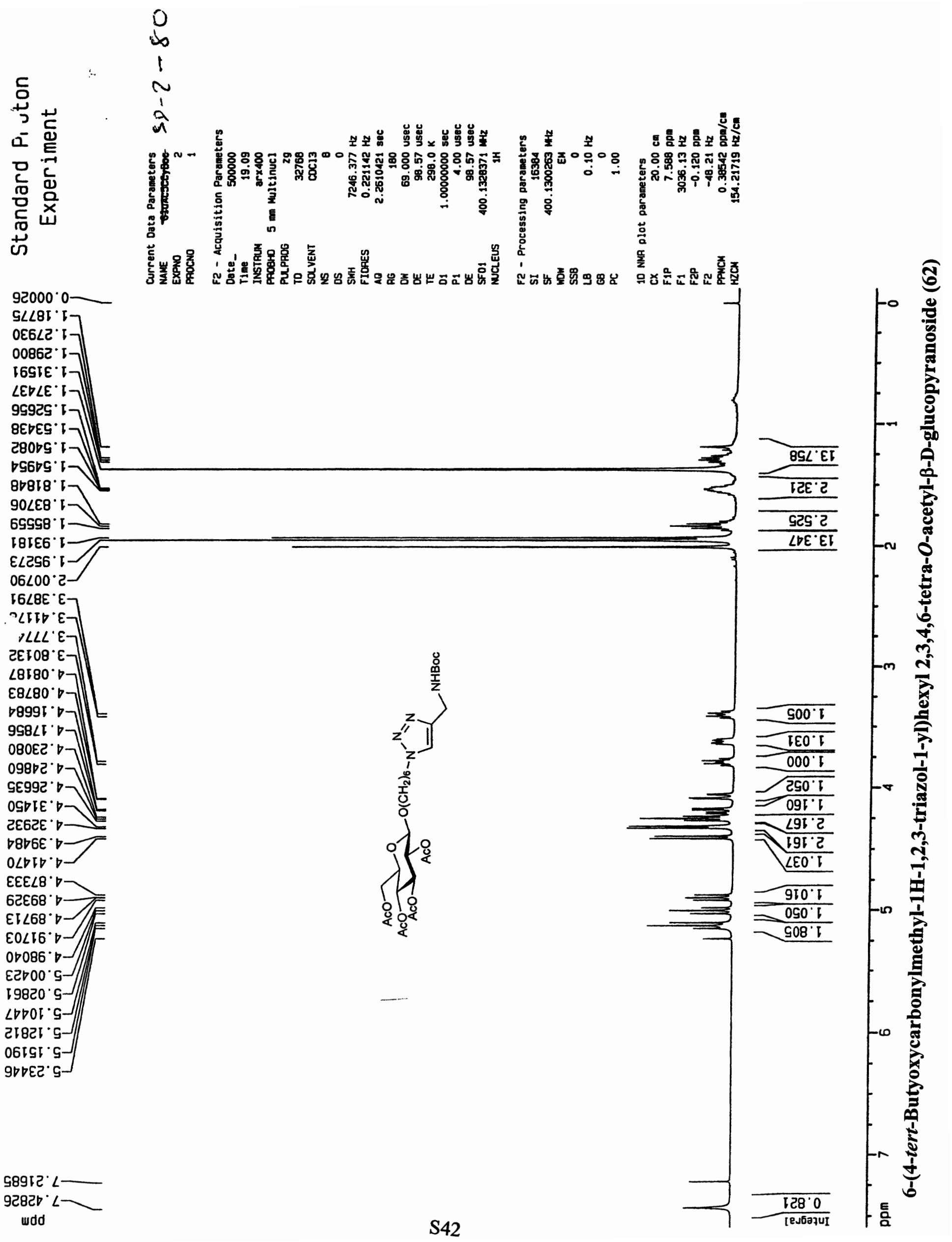




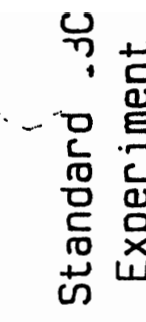

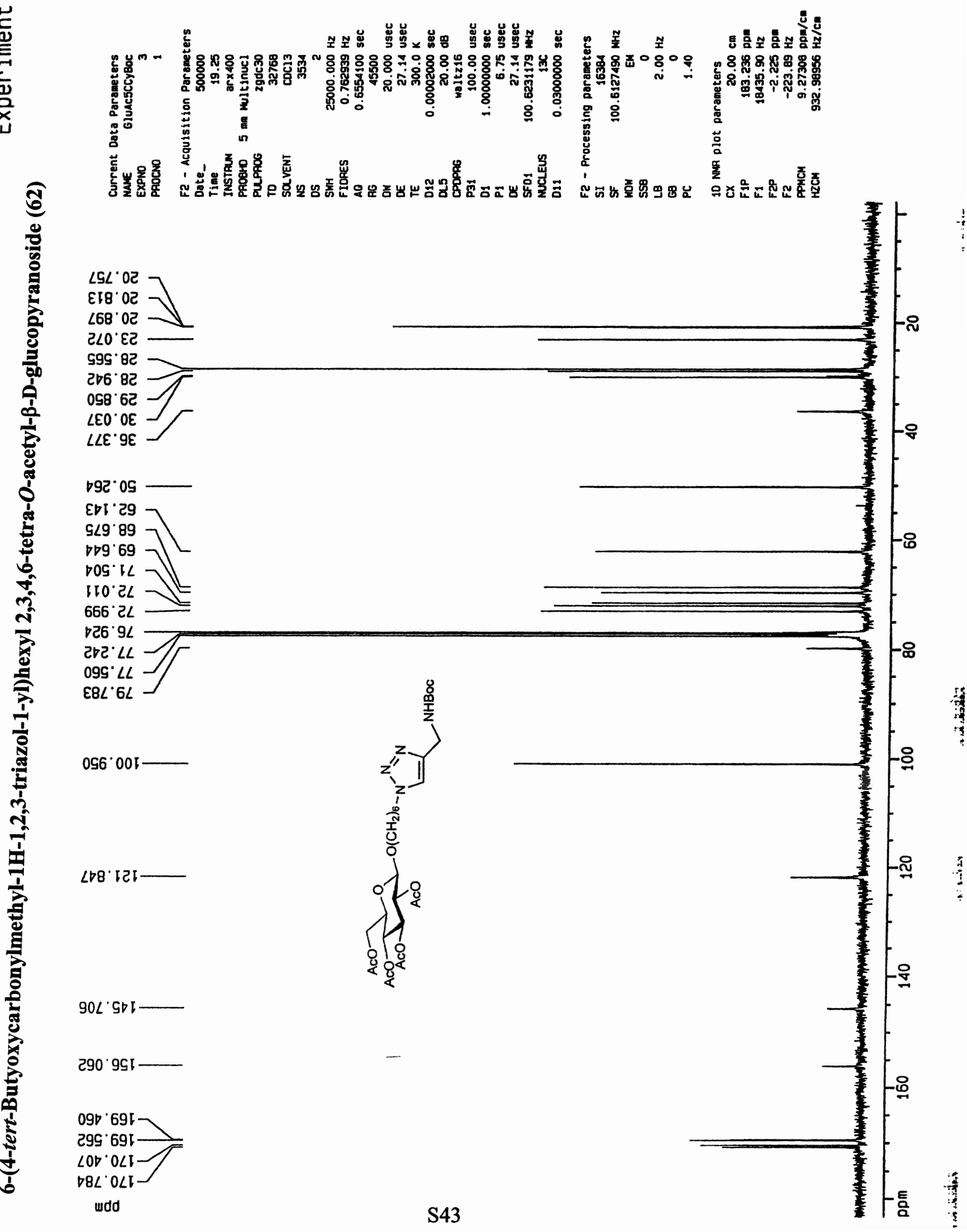



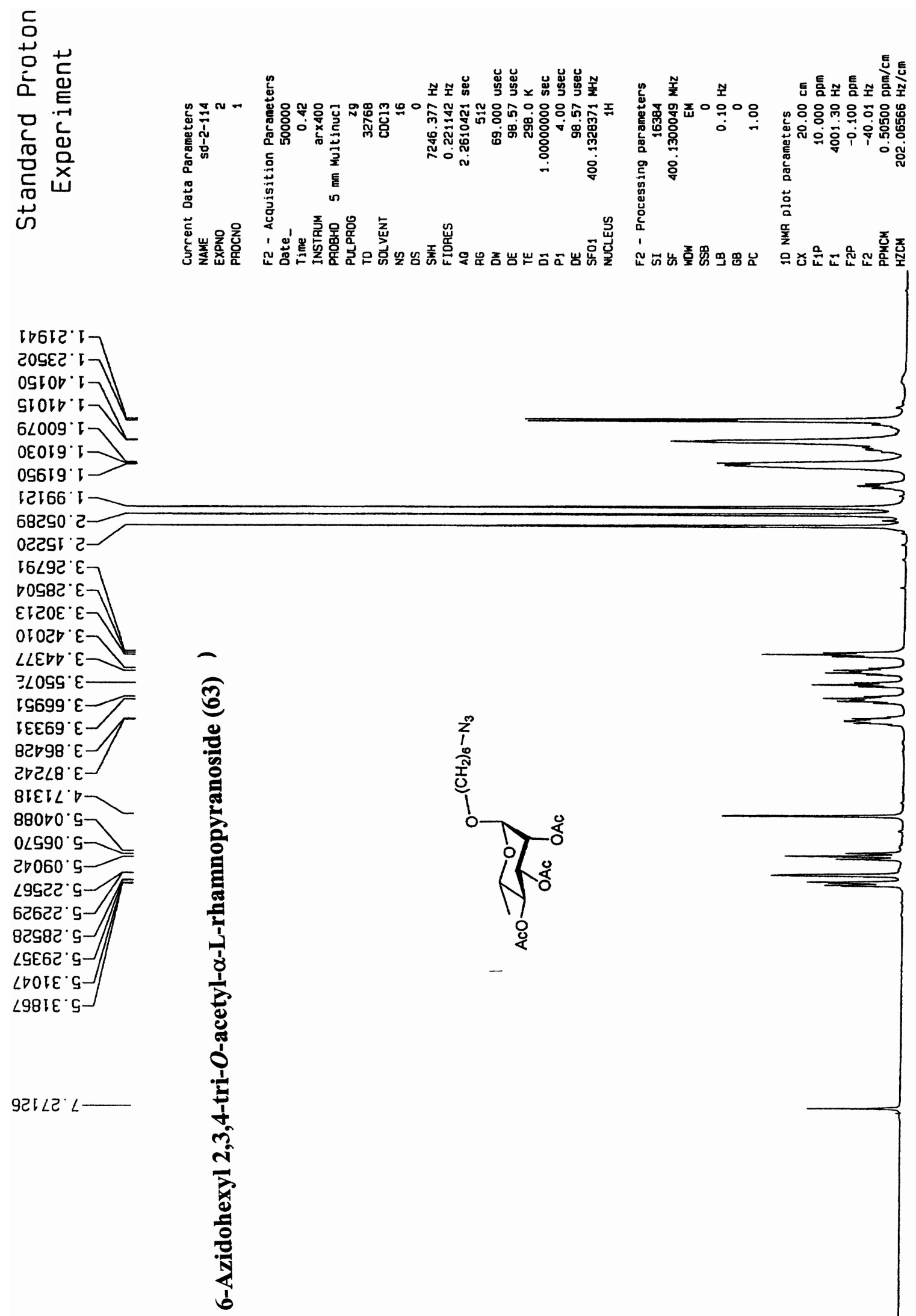

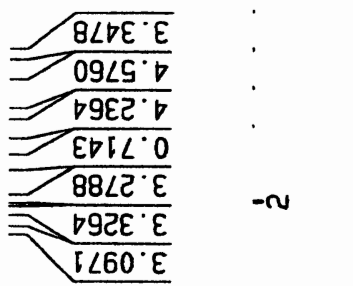




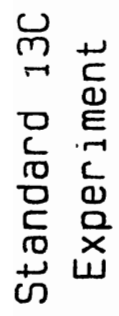
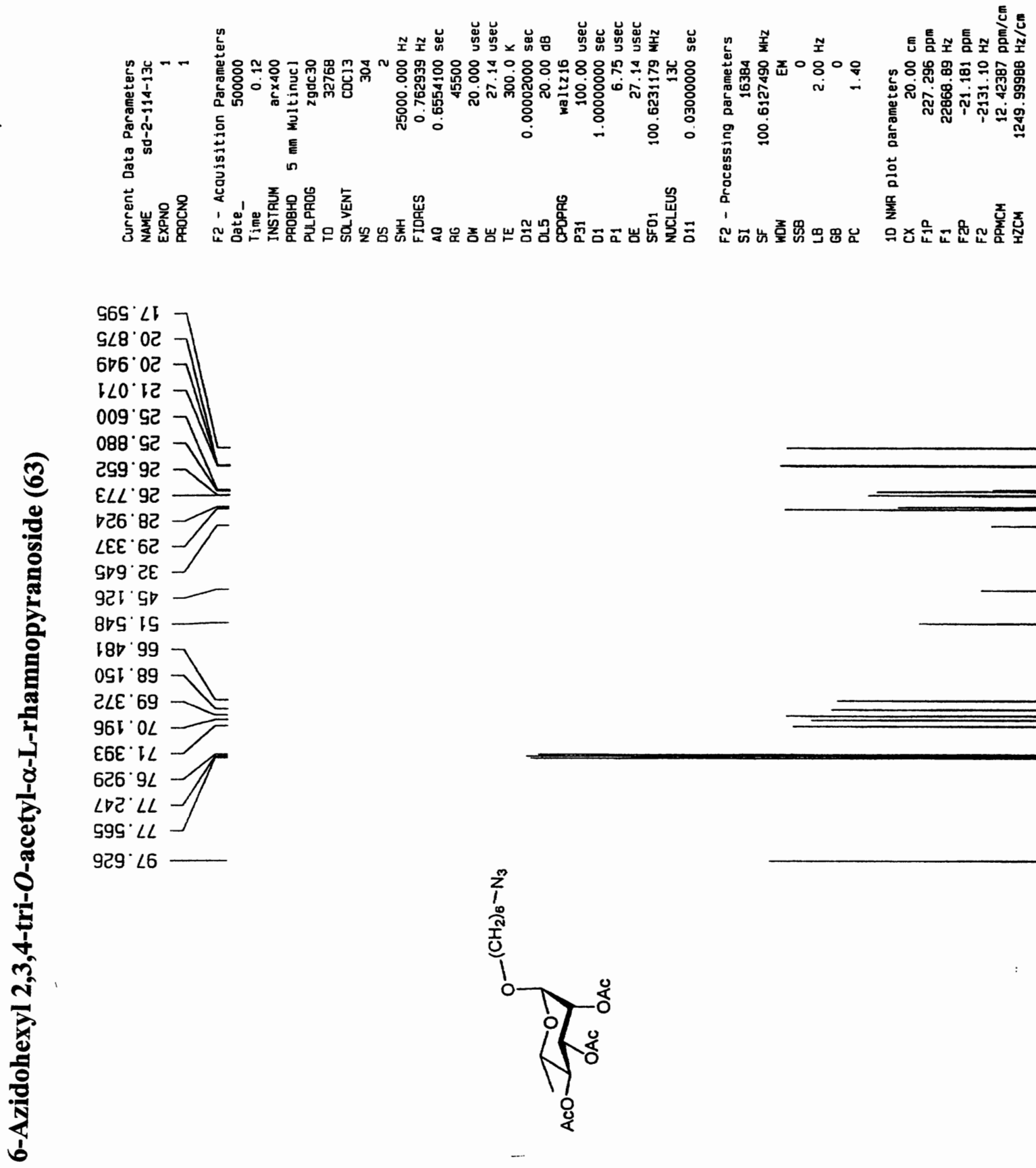

6EI $0 \angle 1$ 8IE $0 \angle \mathrm{I}$

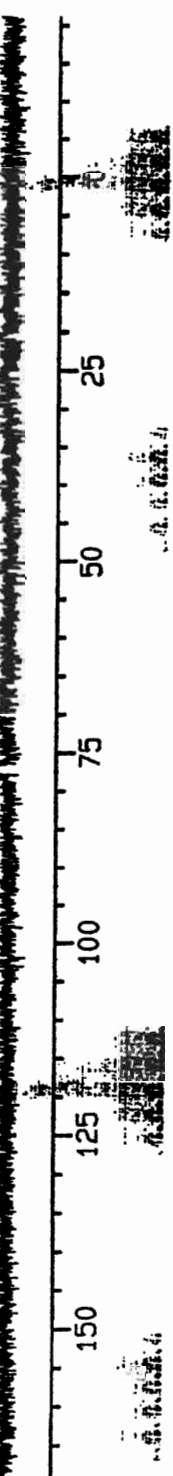




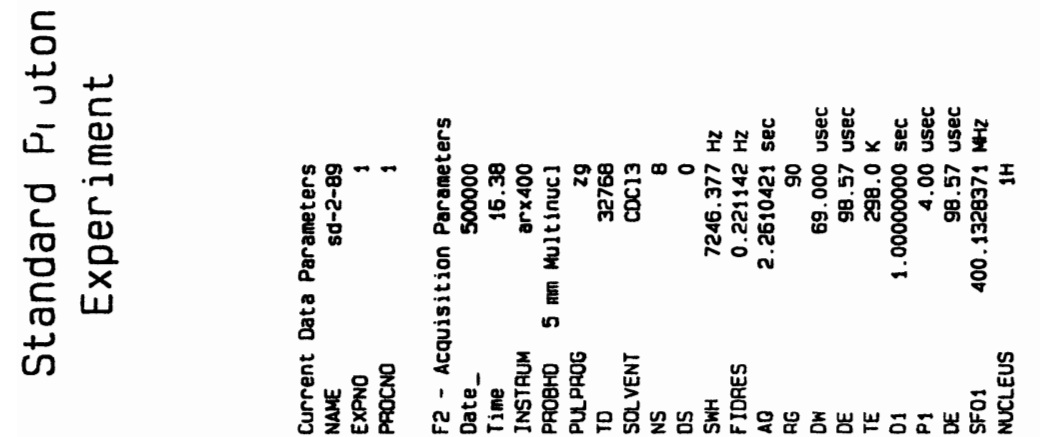

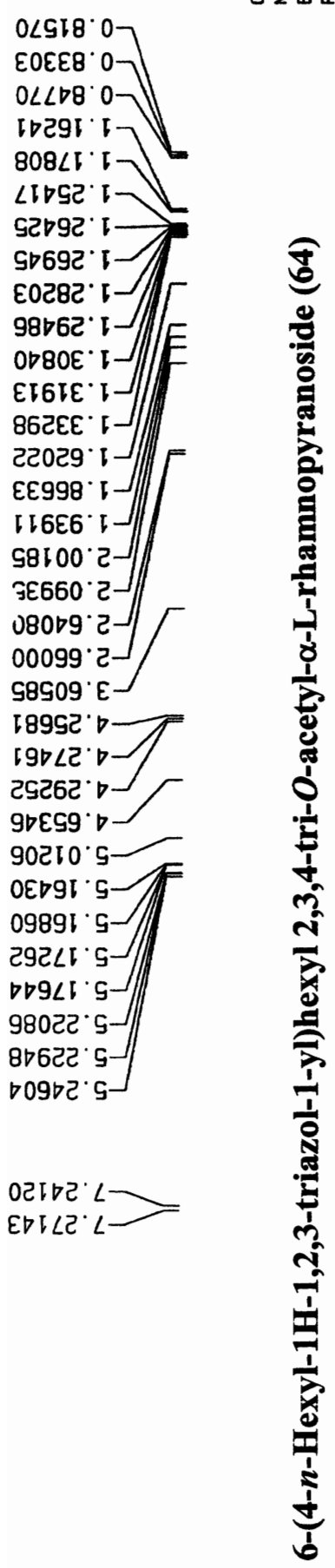
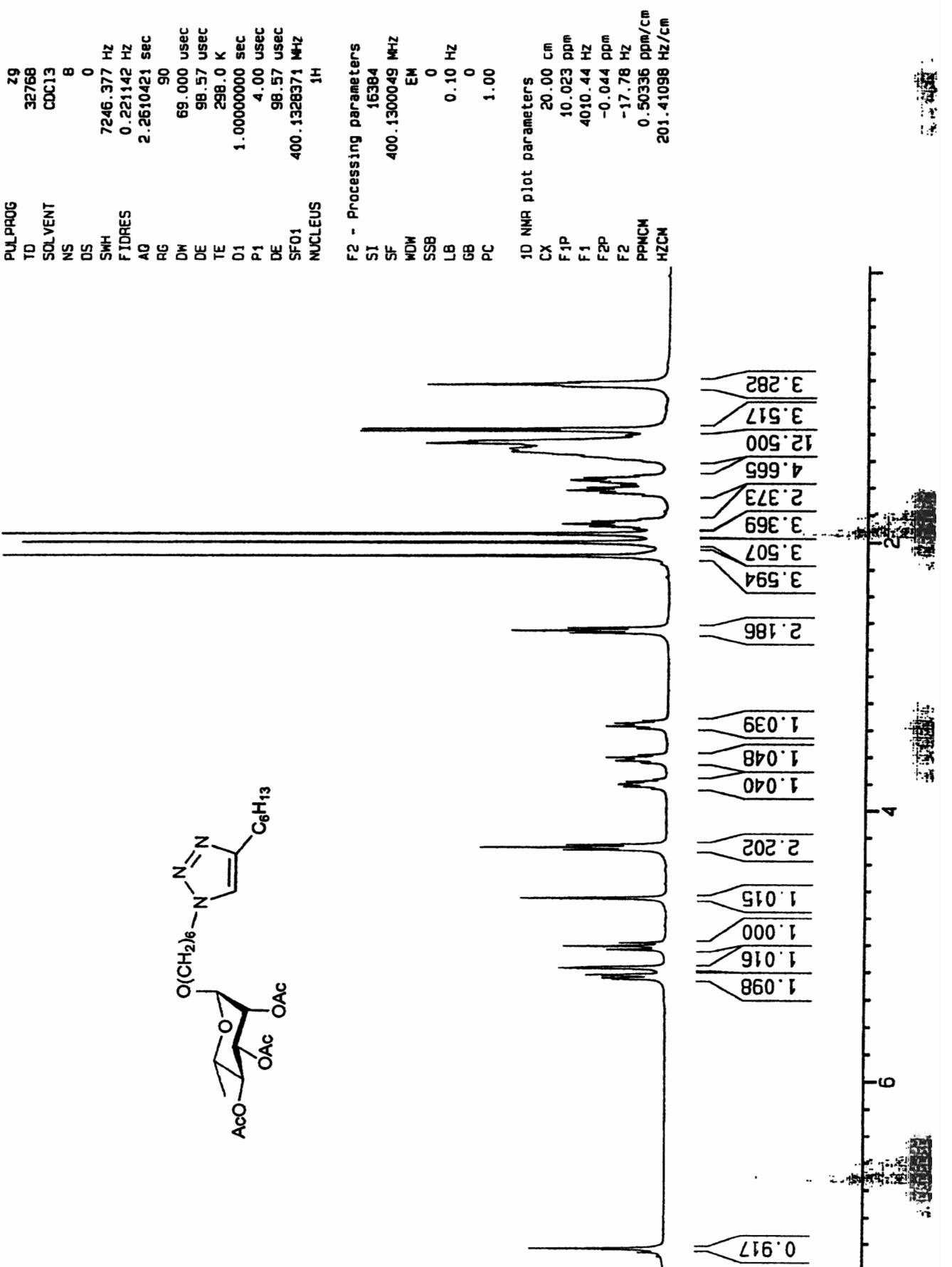


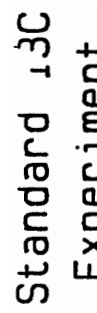
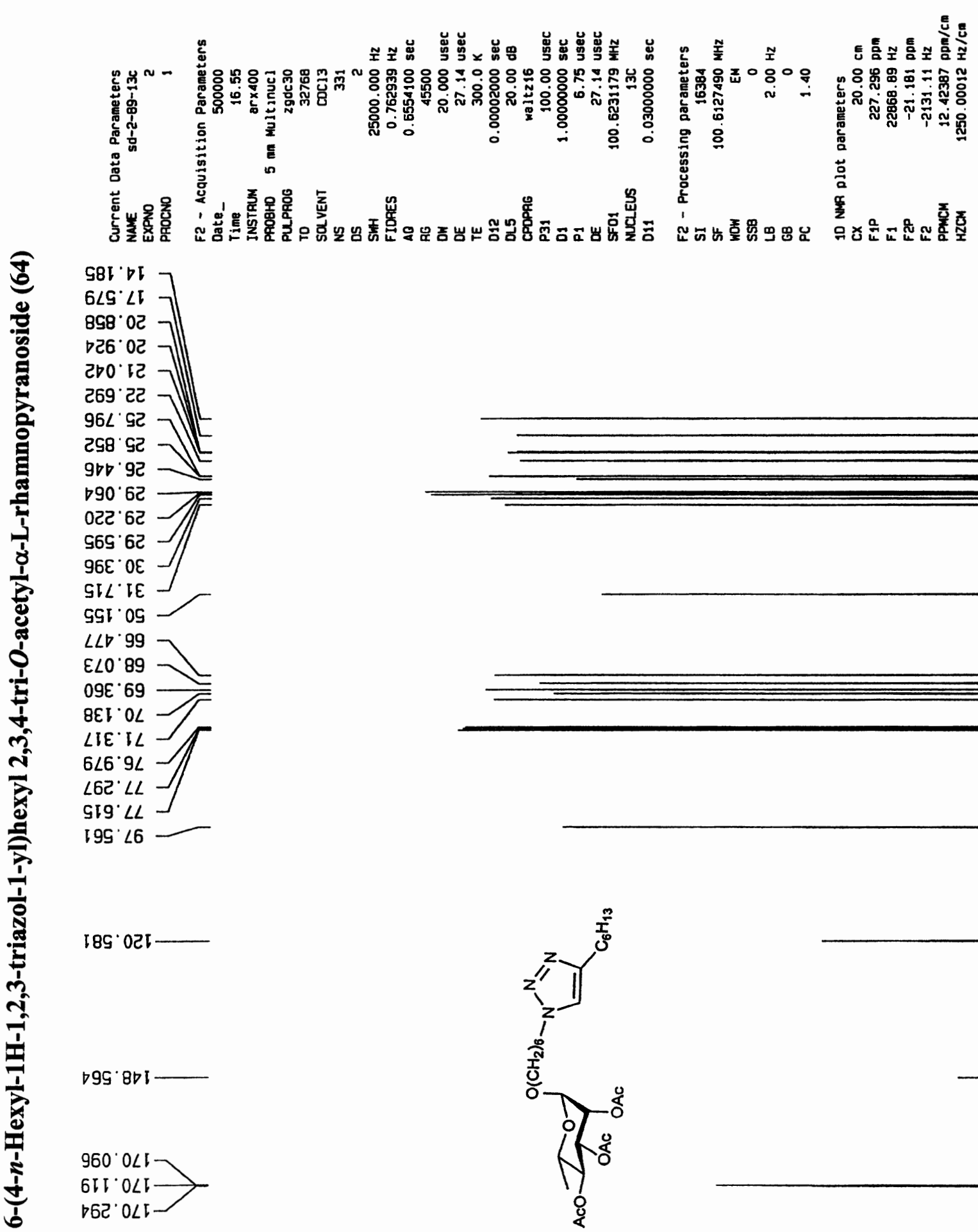

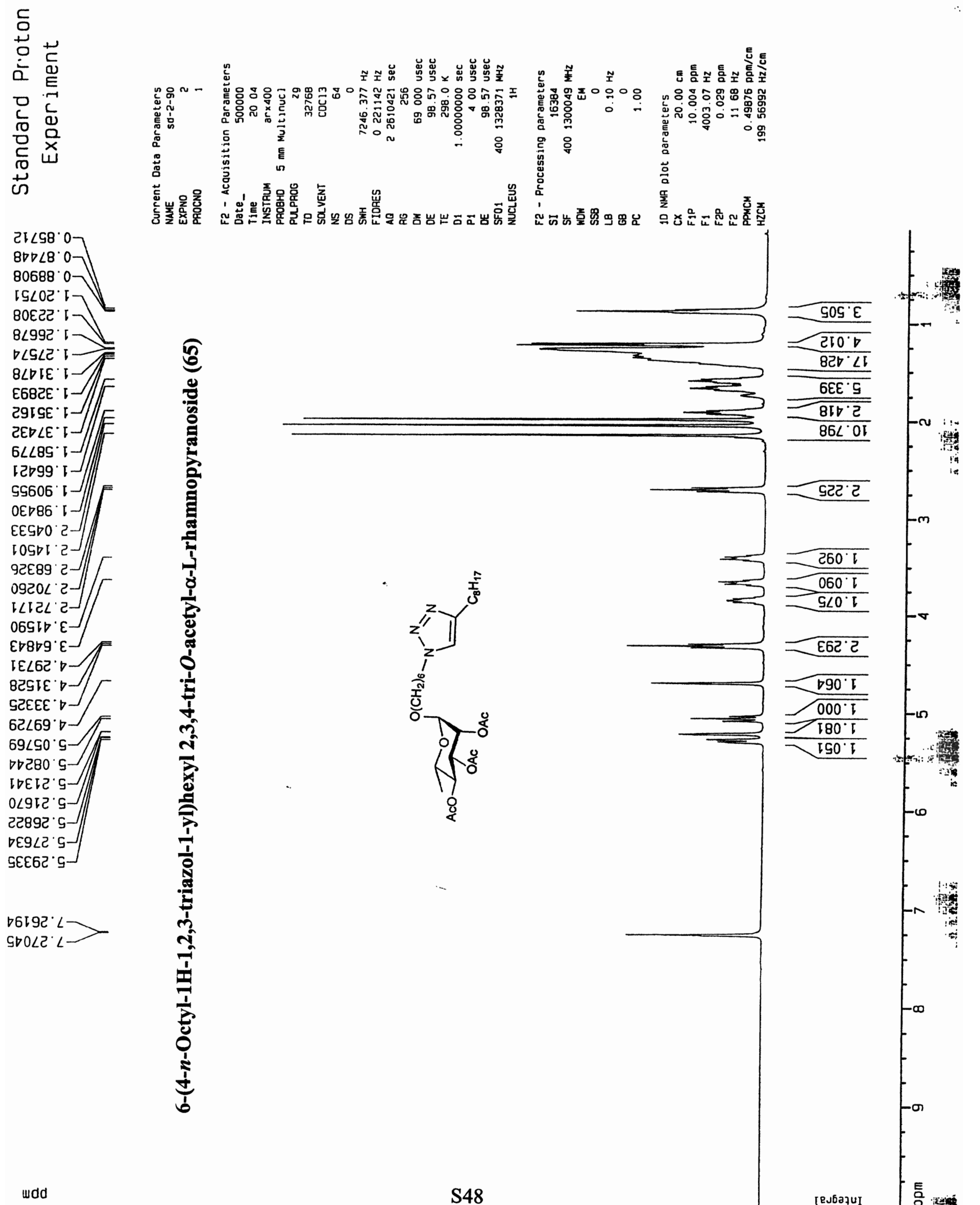

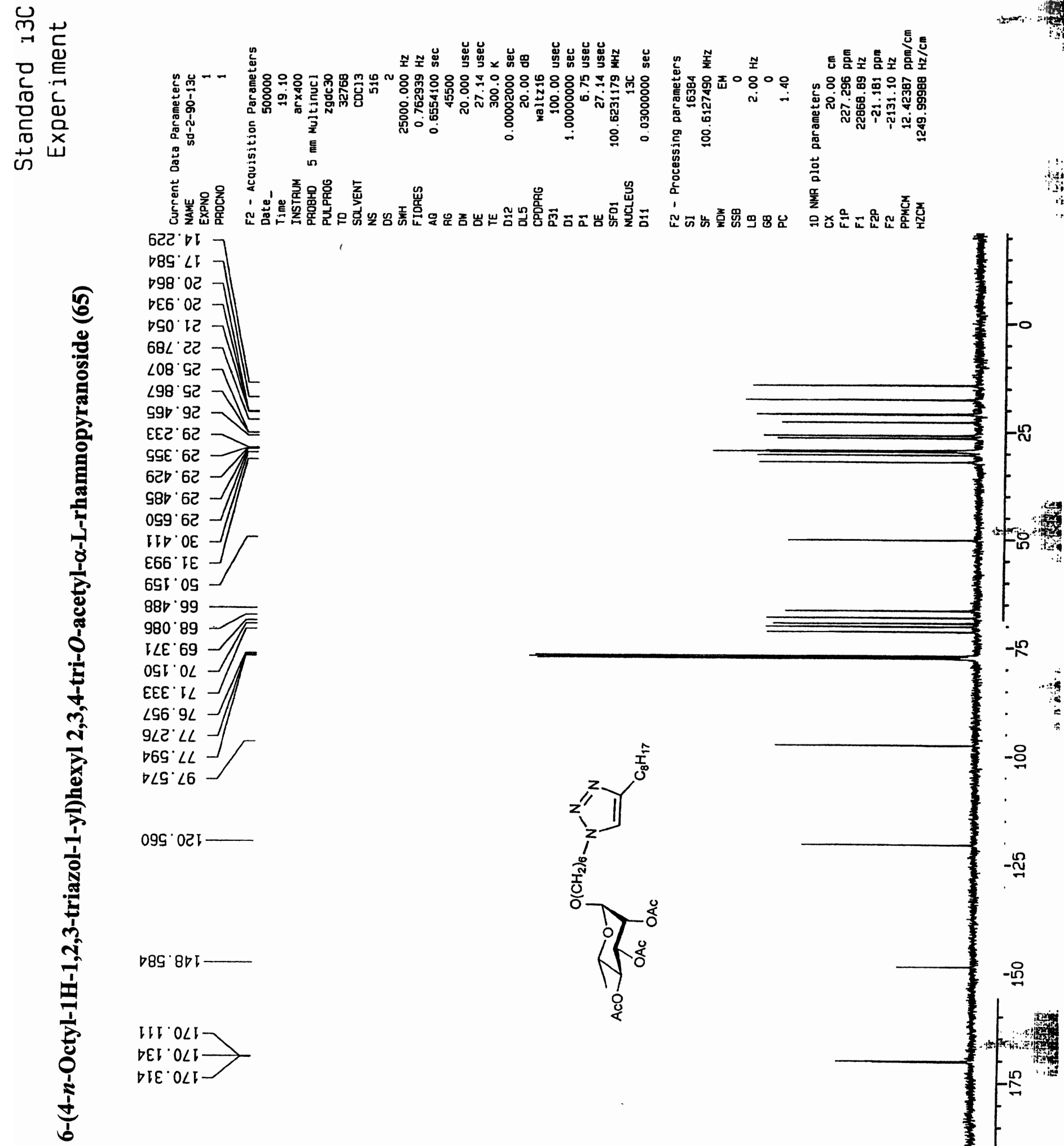

-

- ำ

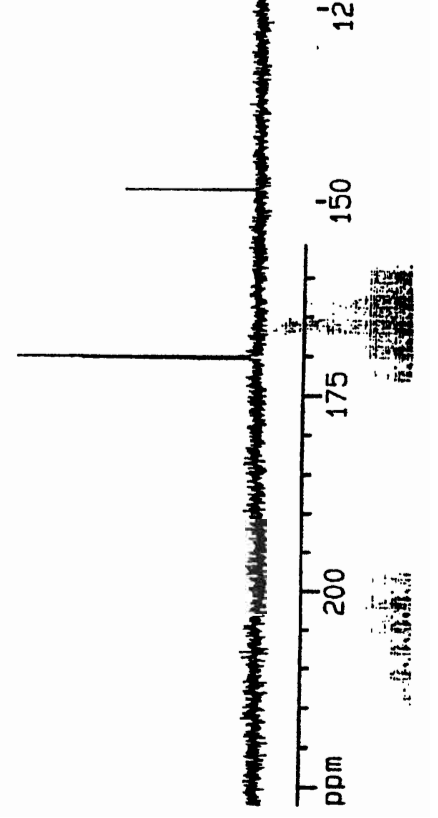




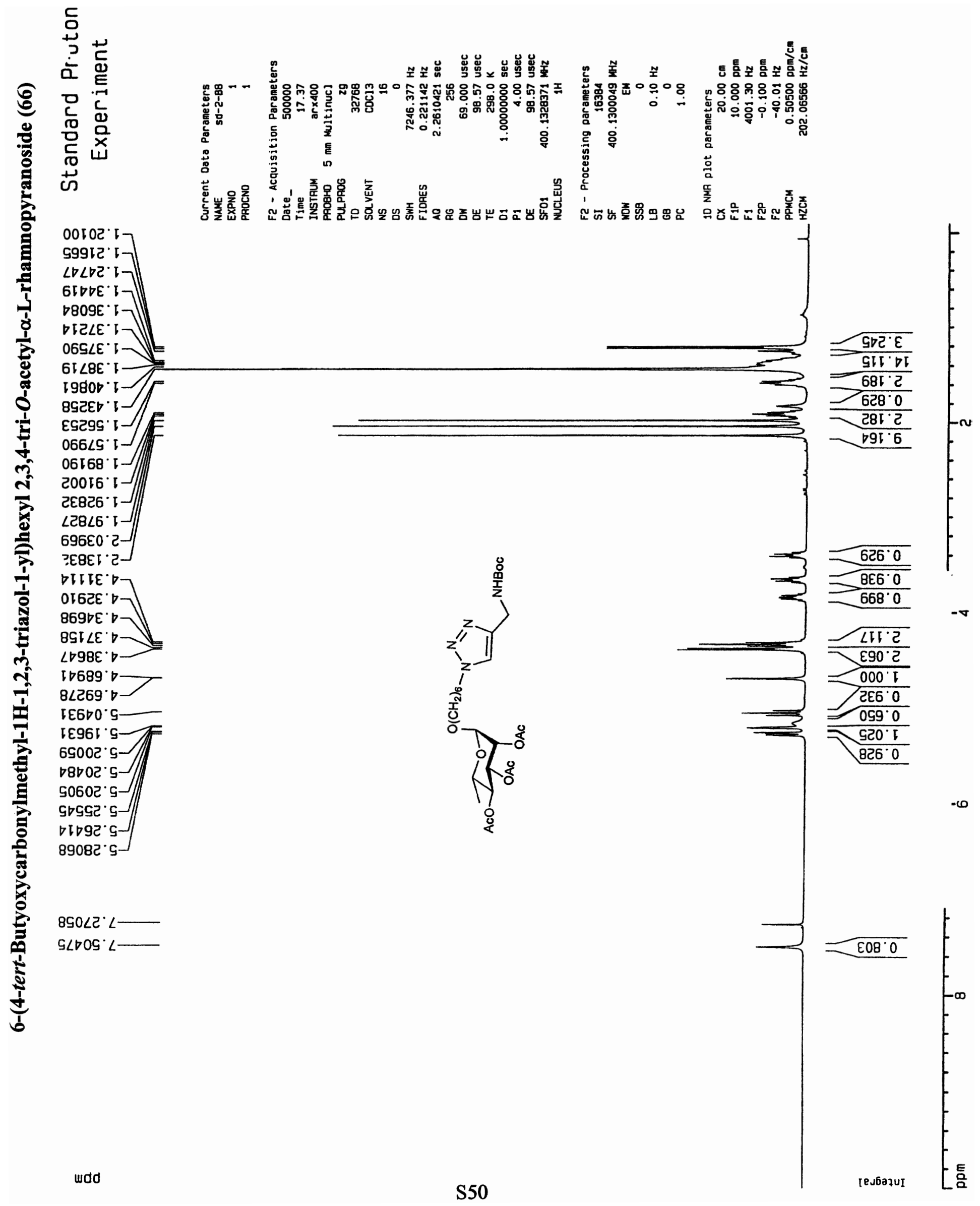




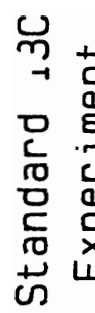

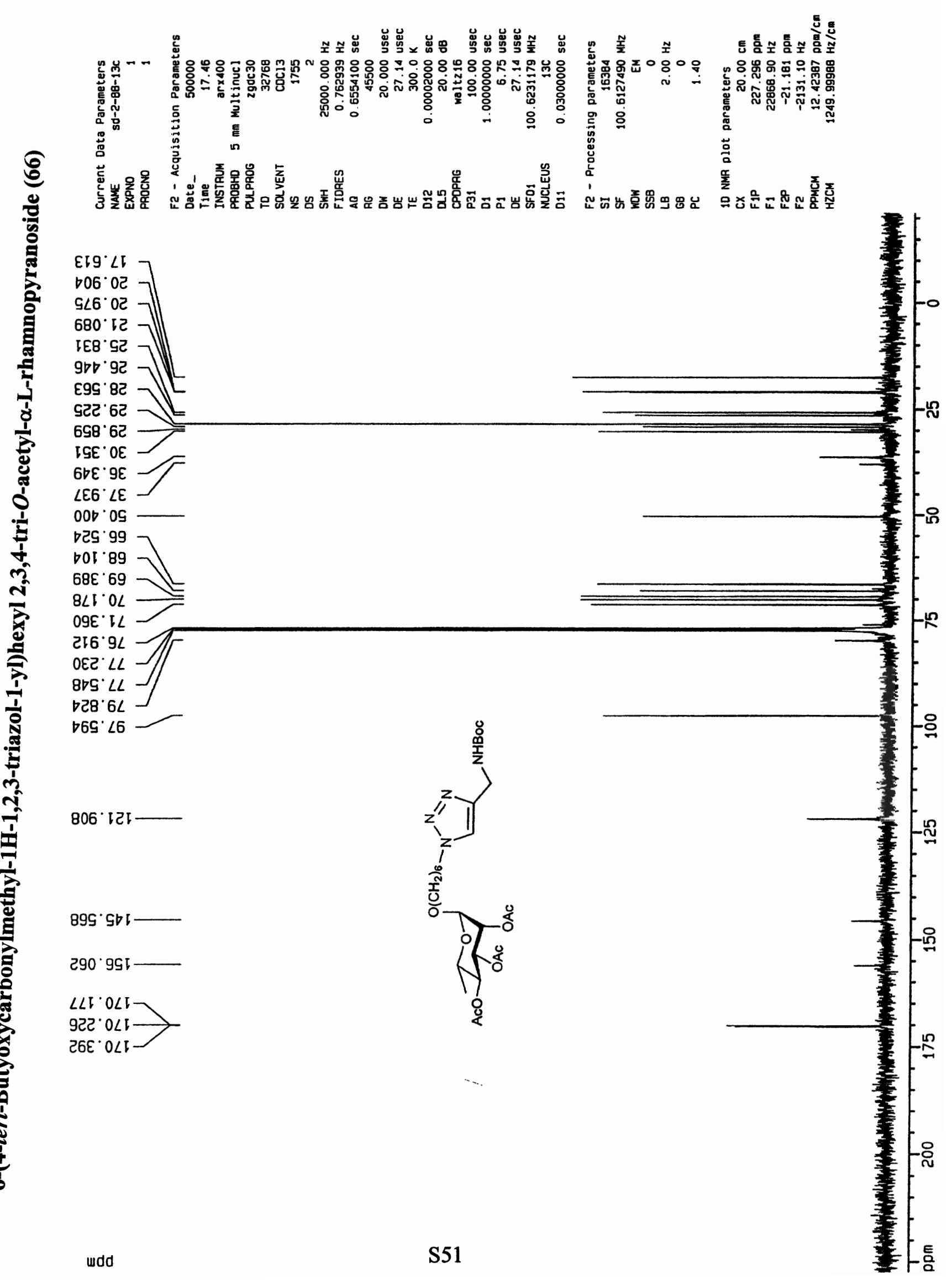




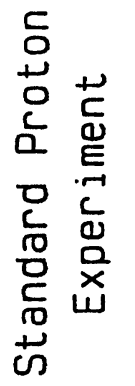
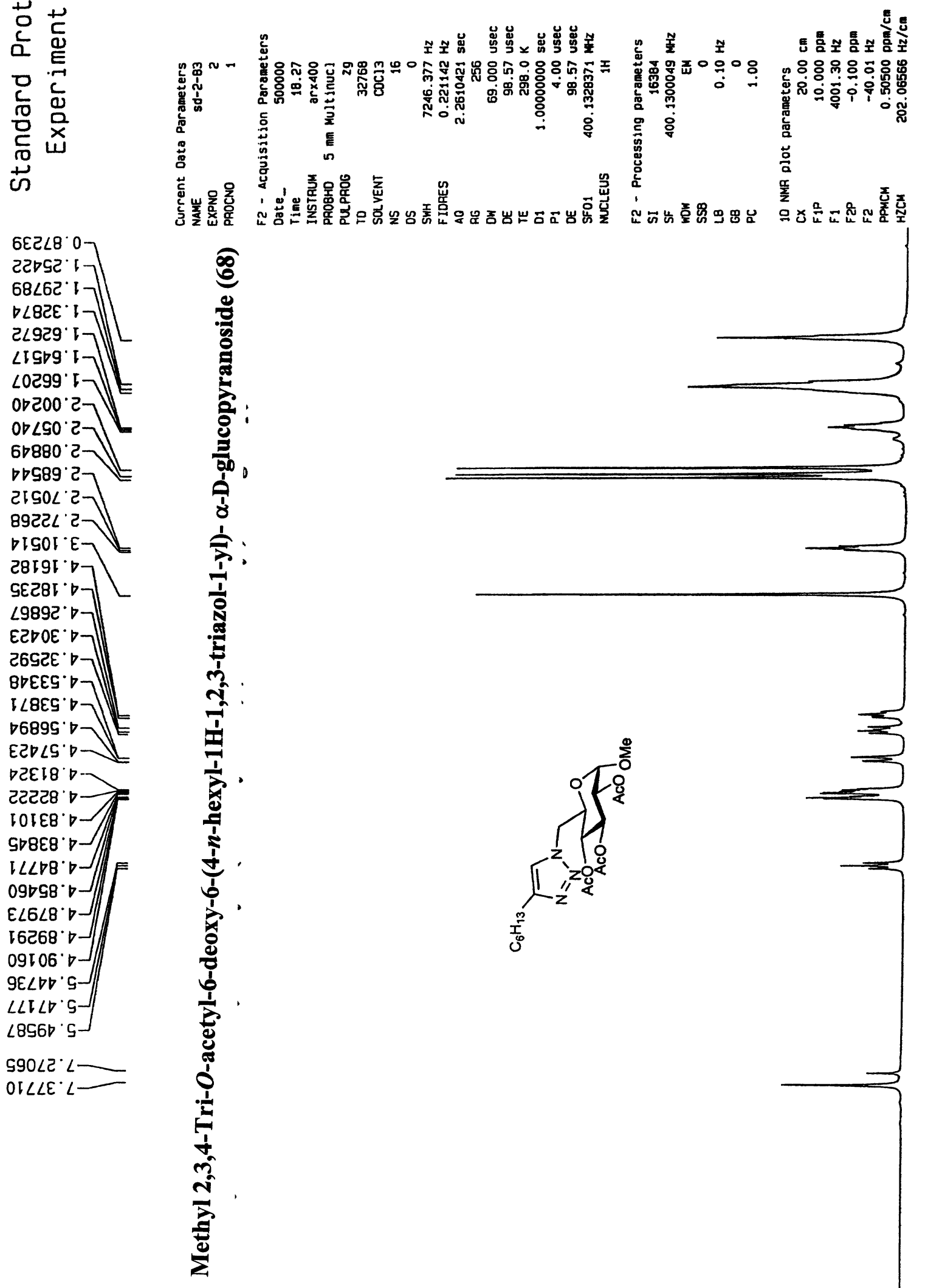

бЕटट8.0 टट口Sट $1-$ $68 \angle 6 C^{\prime} 1$

$\checkmark \angle 82 E$ टL959 $]$ $\left.\angle 0299^{\circ} 1\right]=$ Ob200 $\triangle \nabla 989^{\circ} \mathrm{C}$ टा 902 ᄃ SEटBI' $\square$ $\angle 9892$$$
8
$$
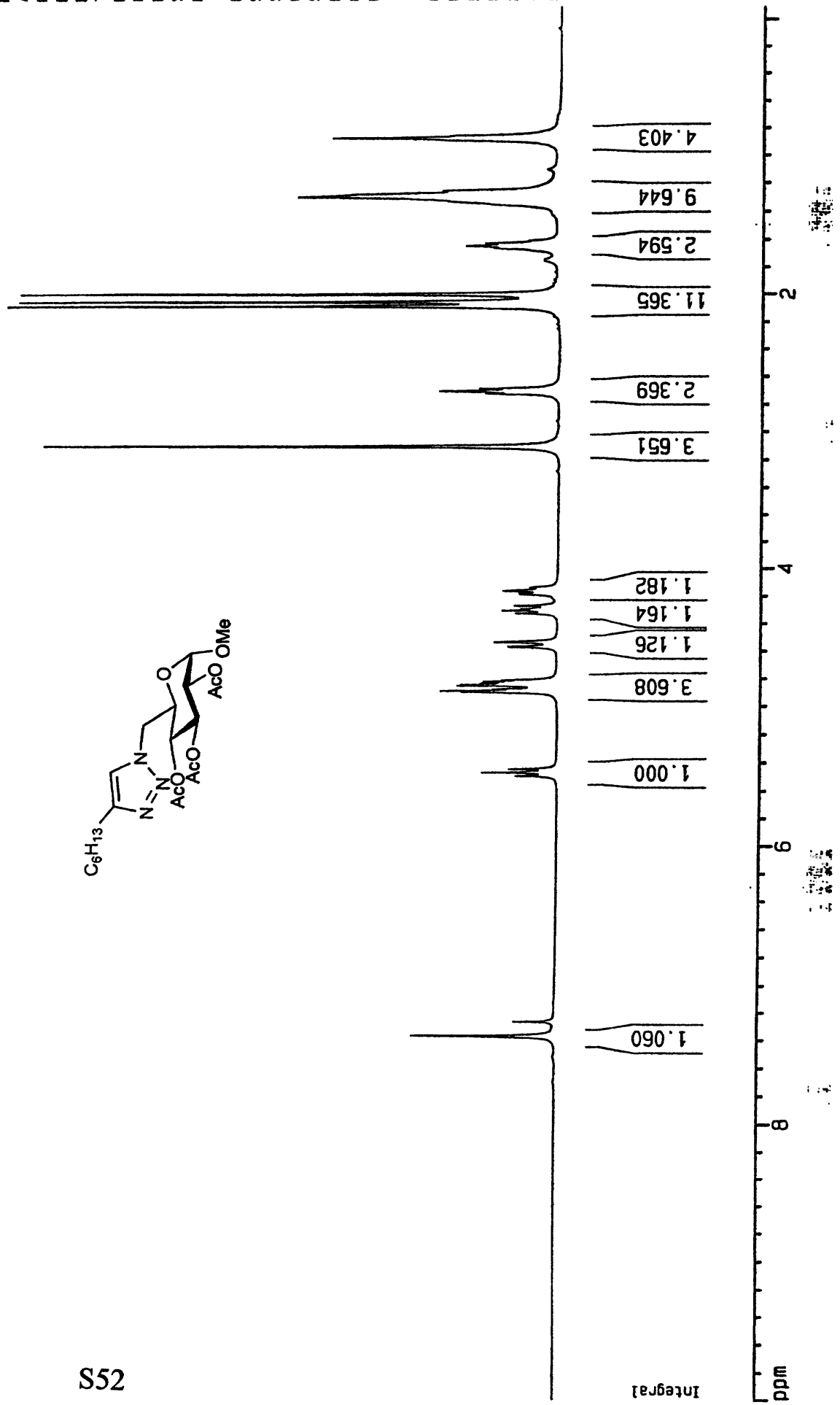

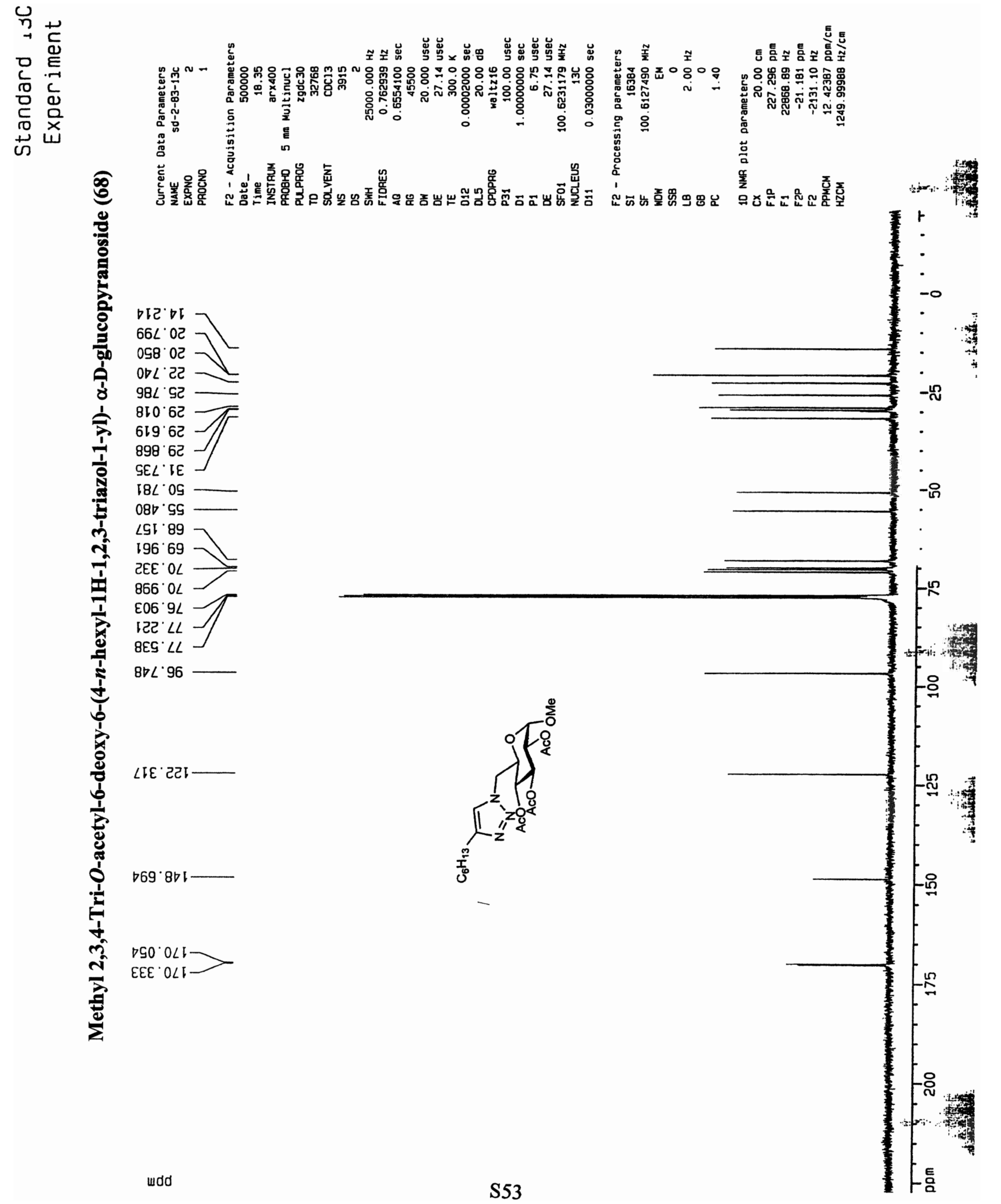


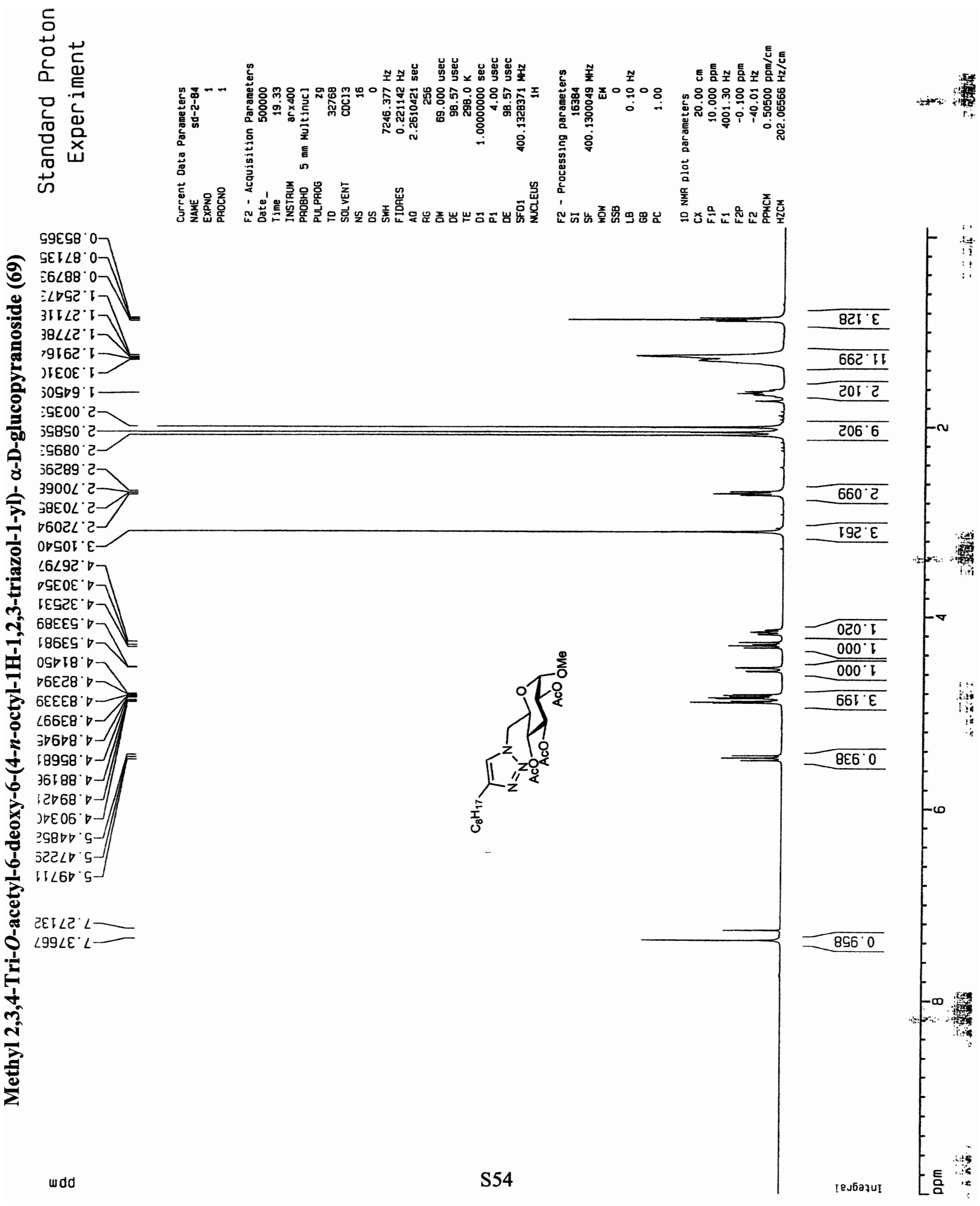




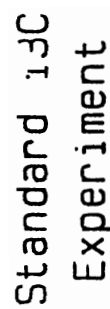

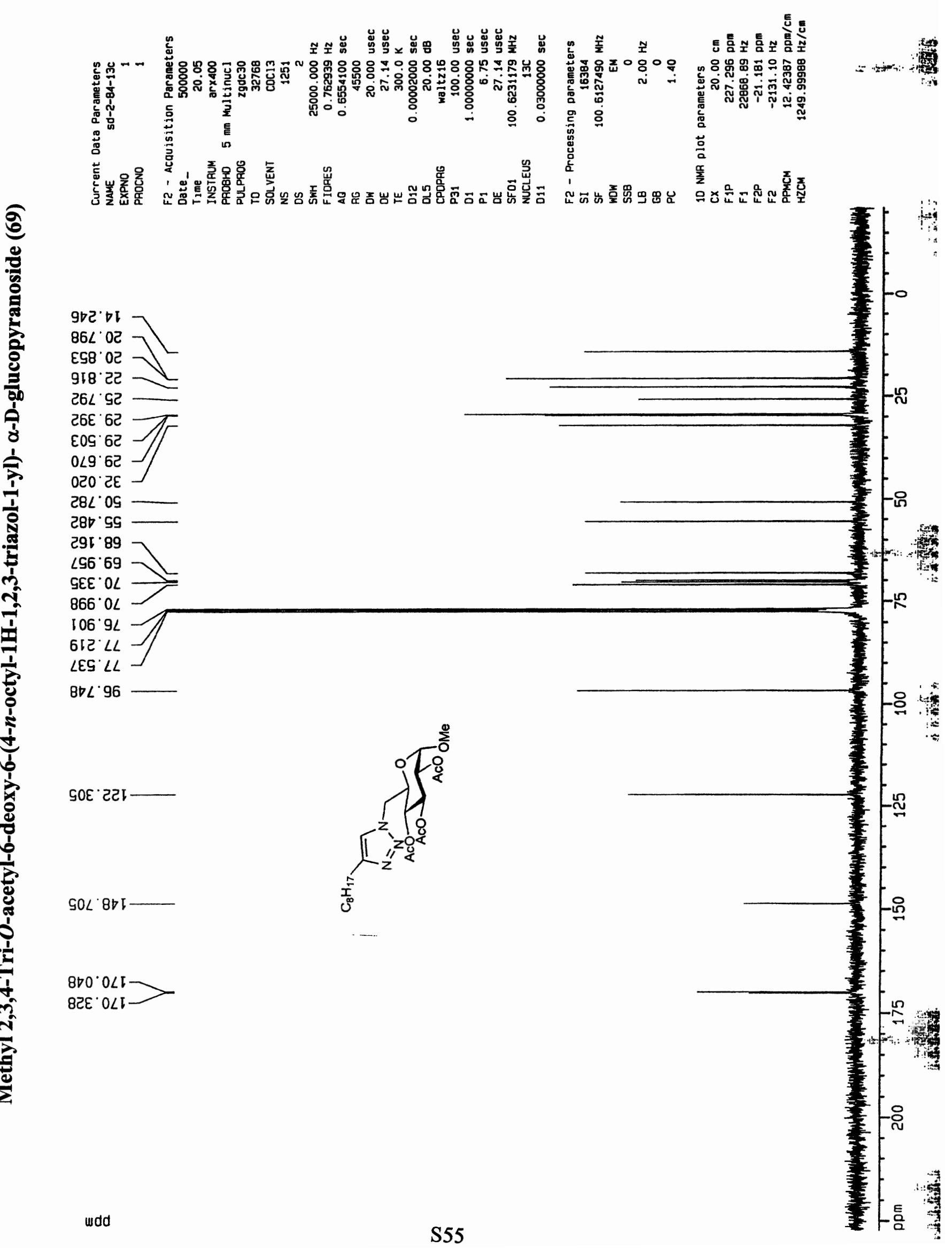



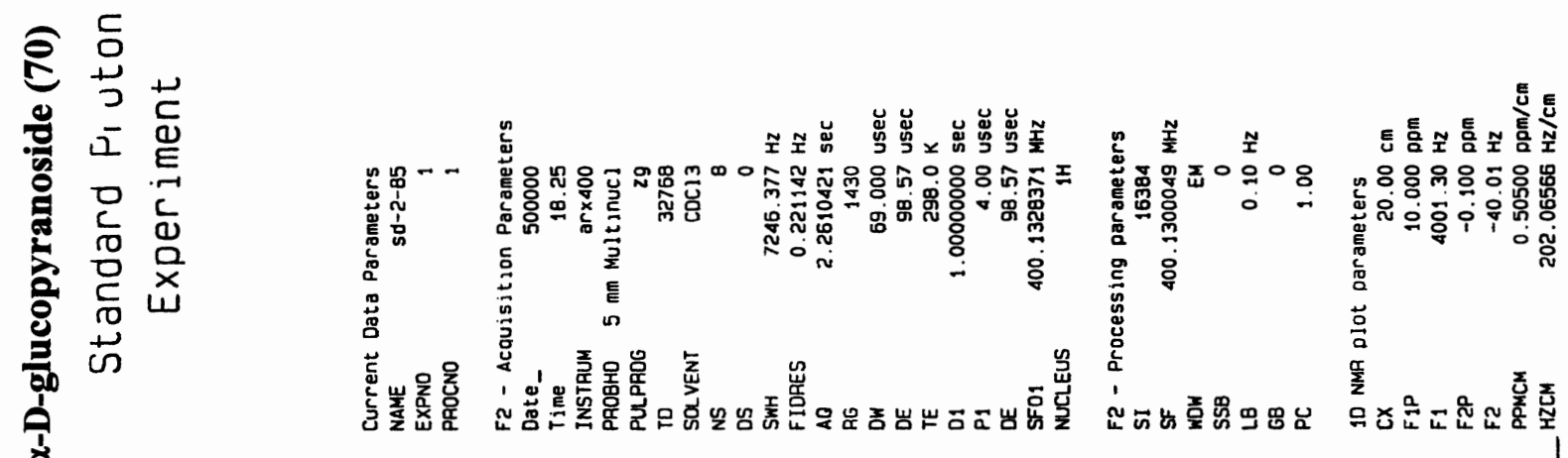

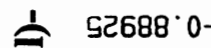

i sig92

टटЕ६ट '

OLटDE

S8टD०. 17

96009. L

O2E $\left.10^{\circ} 2\right]$

$520 \angle 0^{\circ} \mathrm{C}$

EOSOL

टह6६I.

$88978 \cdot E]$

\section{IEE L $T$}

LCSटE $\square][$

उ०L9E

jOD $8 E^{\circ}$

6E66E

899bs

\section{EEटSG $\nabla>$}

BEटZS

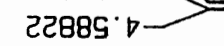

$66128^{\circ} \cdot-$

$\triangle \triangle D 28^{\circ} \circ-$

9ODEB $\cdot \square-$

$8 \nabla S b 8 \cdot \square-$

$60658 \cdot \square-$

$010 \angle 8 \cdot \square-$

$01006^{\circ} \circ-$

$91606^{\circ} \cdot$

D095b 5

$666 \angle b \cdot s-$

sgros's]

EEI $\angle Z \cdot \angle-$

250096
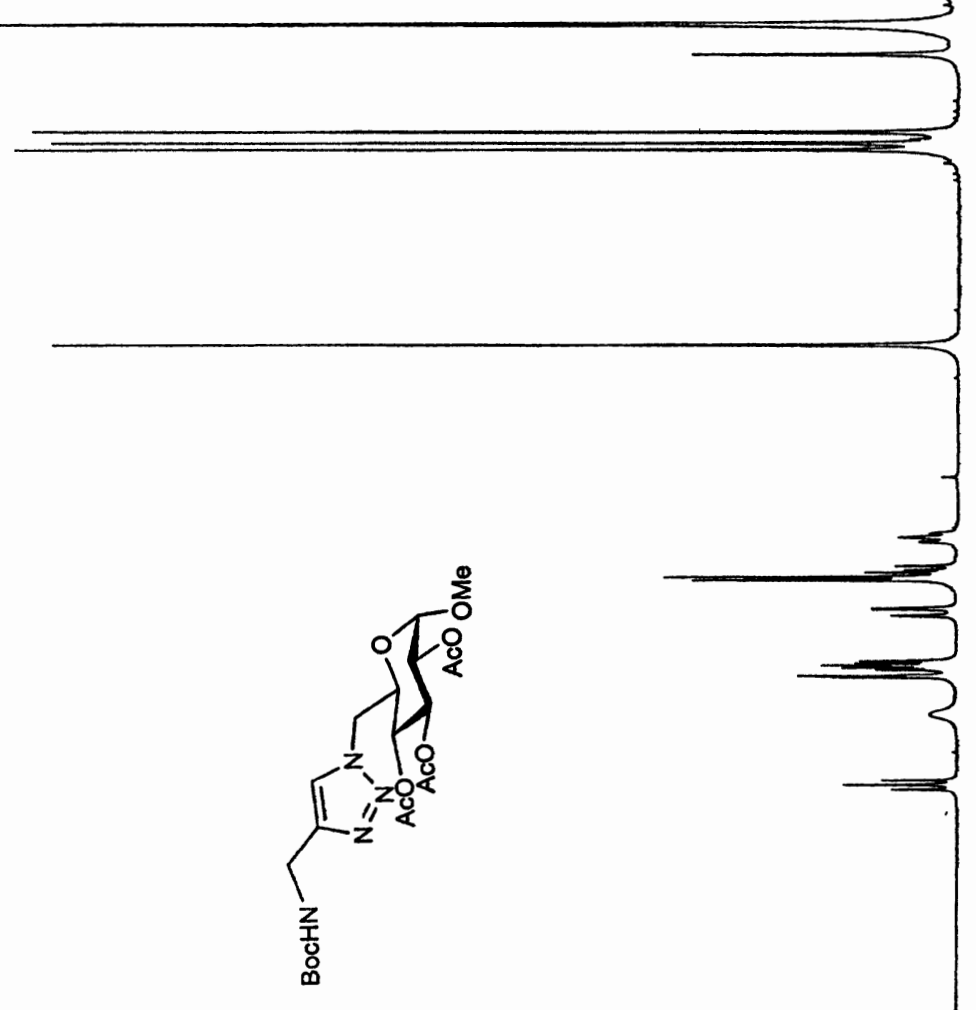

$=0 E 0.11$
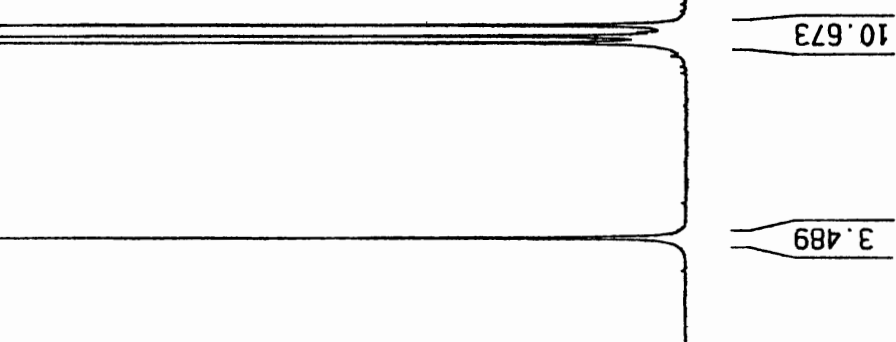

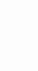




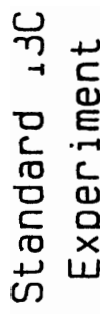

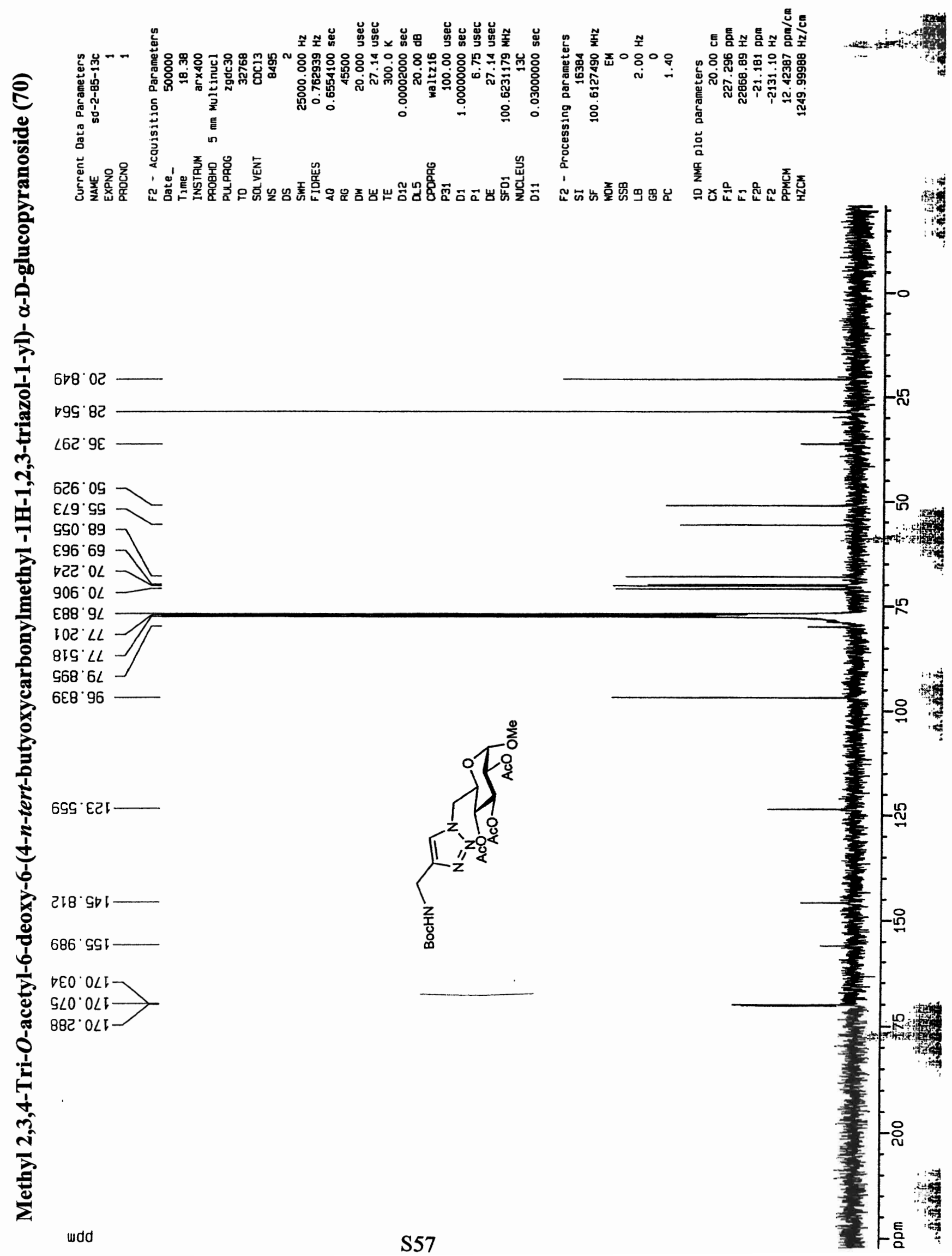



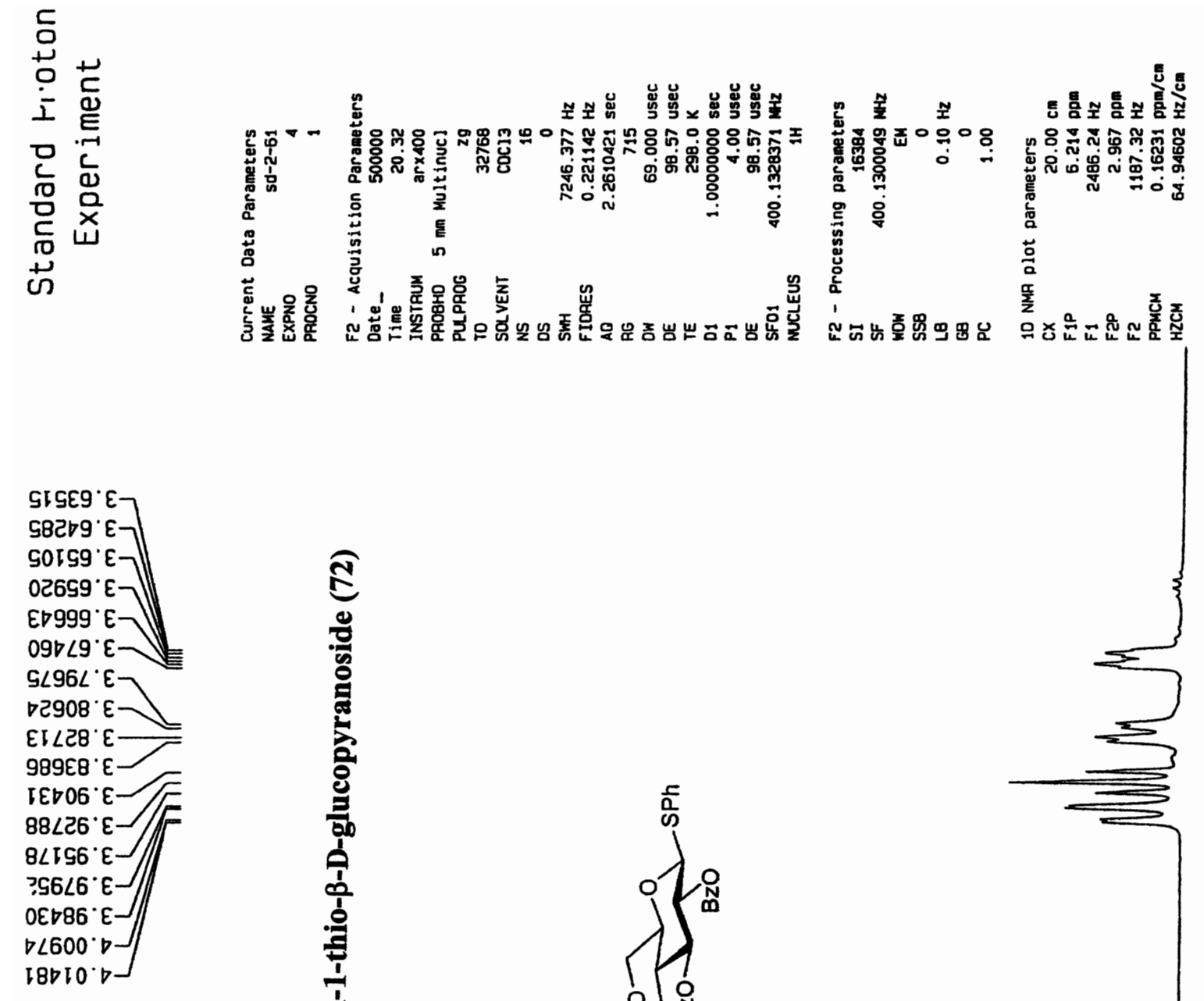

$56209 \cdot$
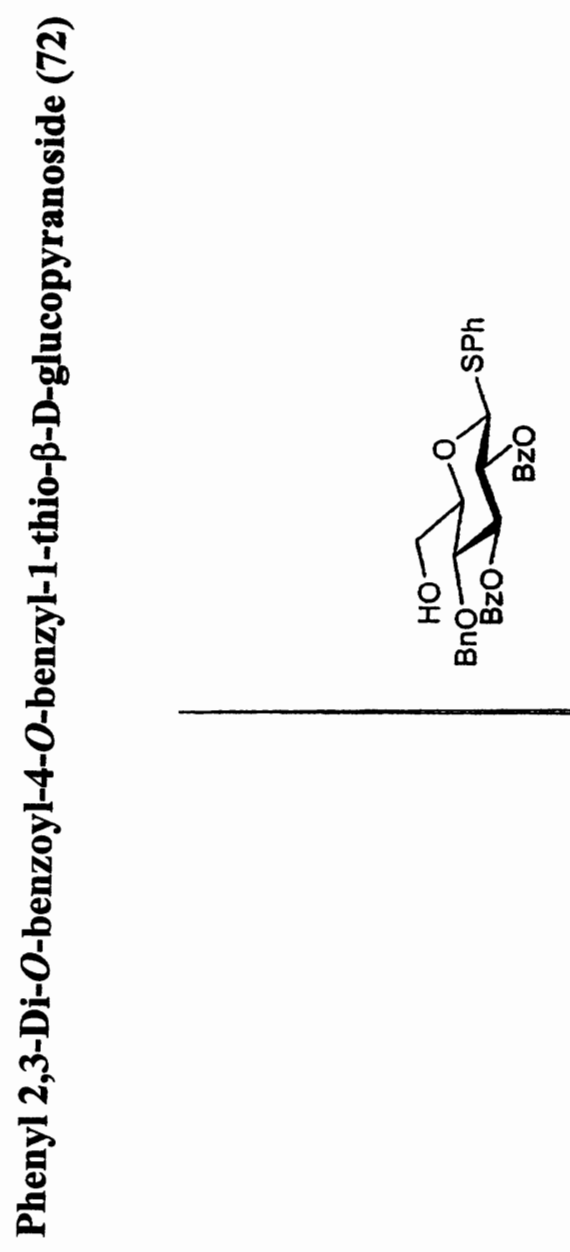

$S 86 \angle 6^{\circ} \circ \square$
$\angle 9000^{\circ} \mathrm{S}$

$\angle 96 \nabla E^{\circ} S$

SLE $\angle E^{\prime} \mathrm{G}$

6E86E.

E08EL'

SDISL'G

$06 \nabla 8<\mathrm{s}$
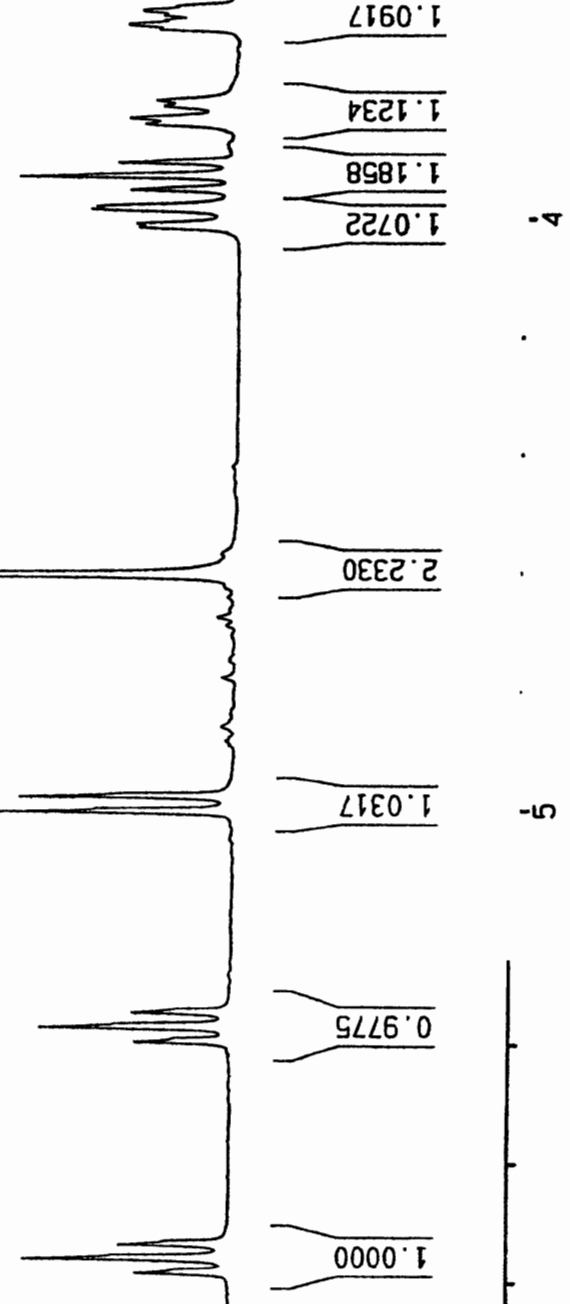


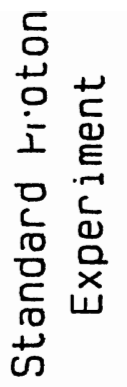
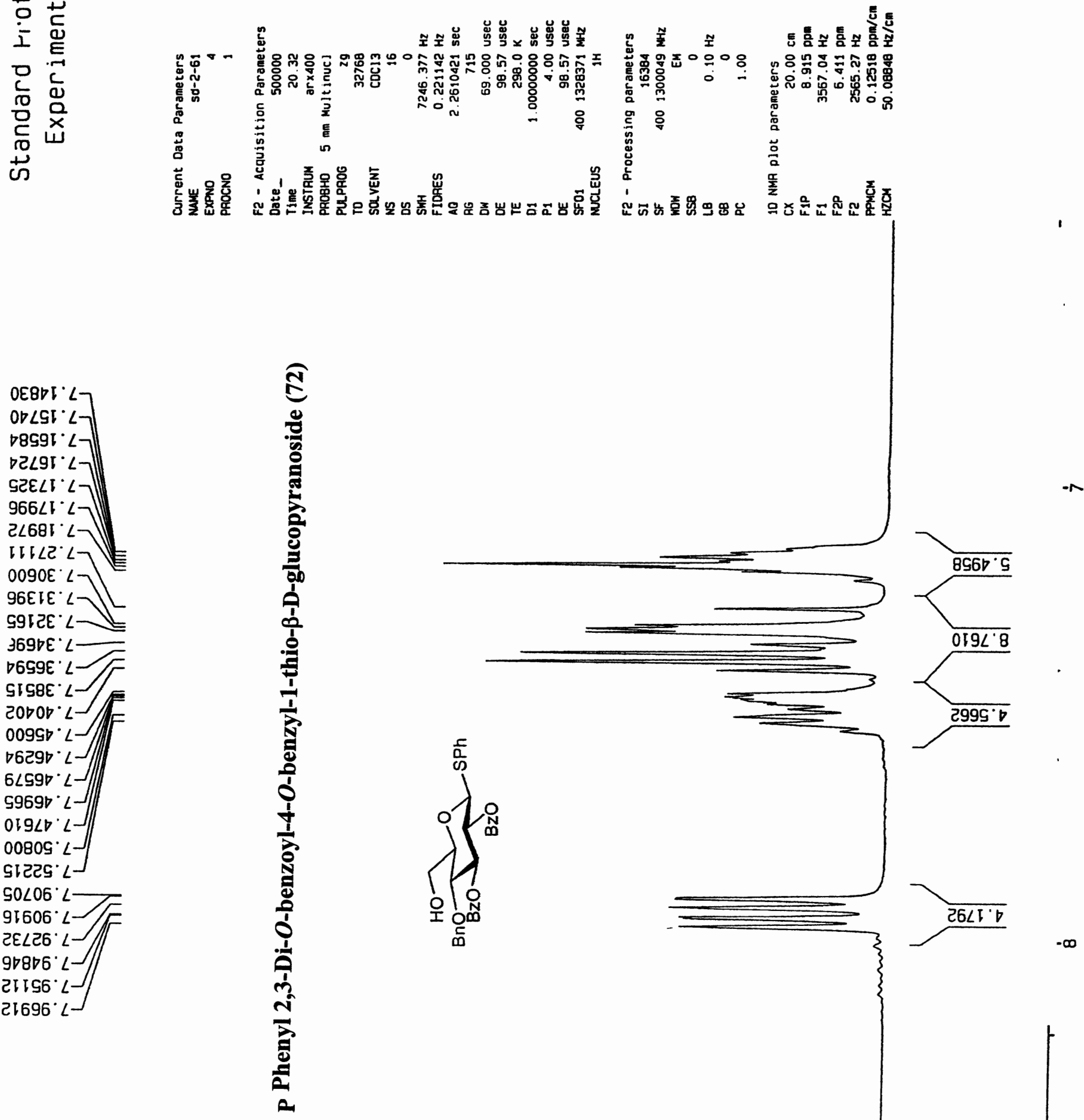

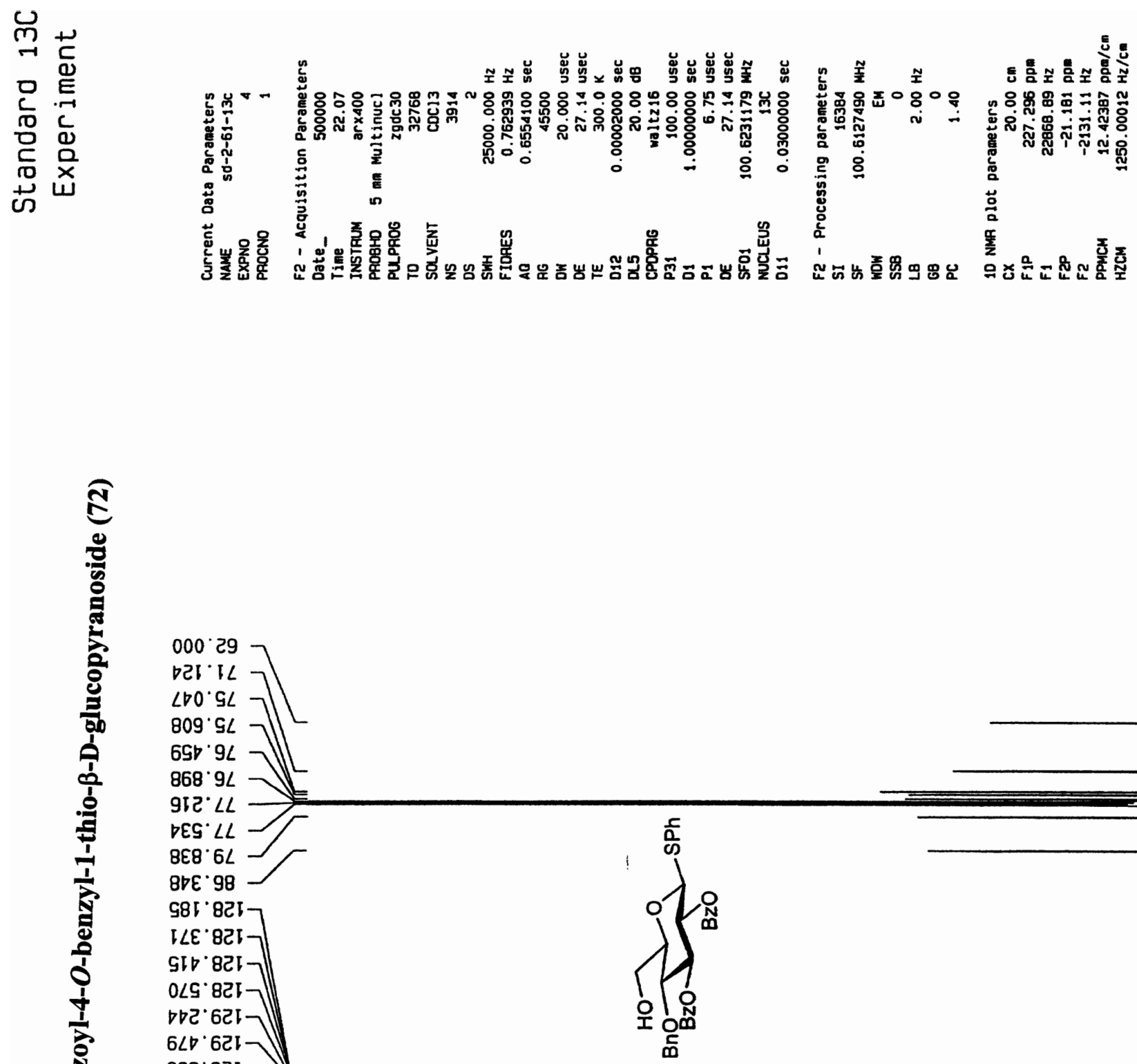


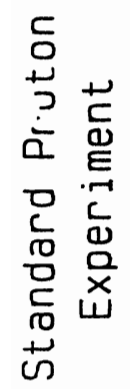
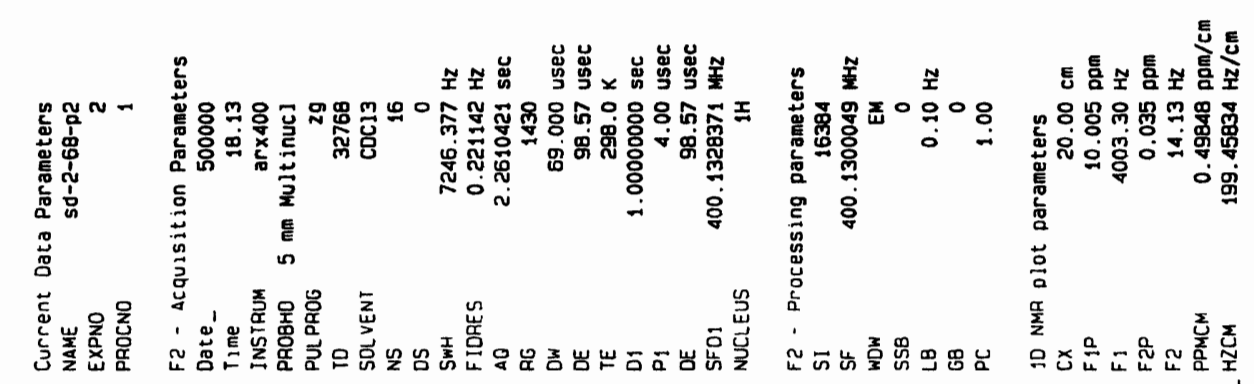

90626:0
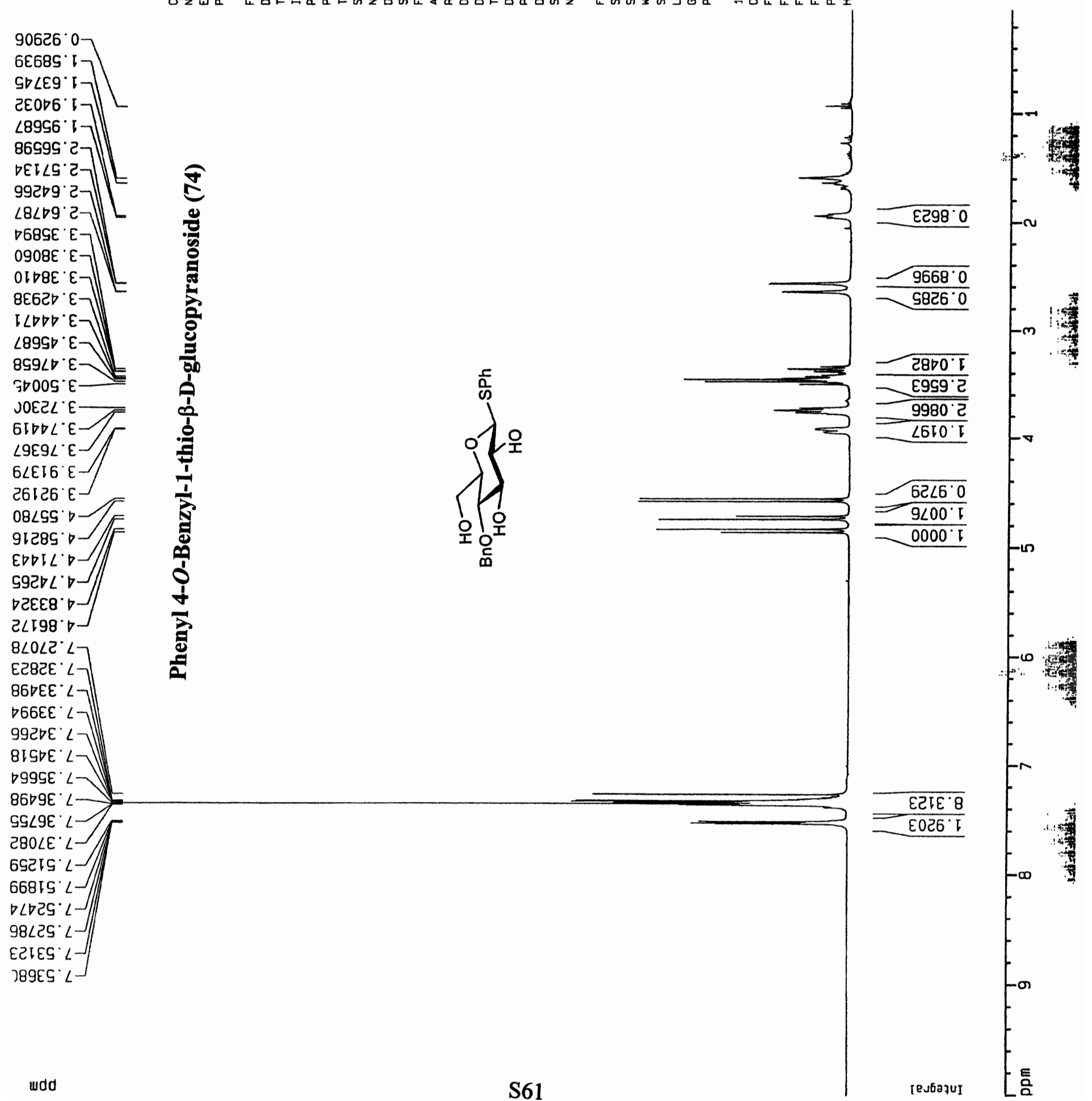


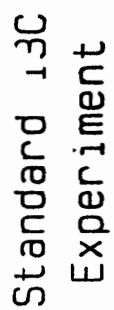

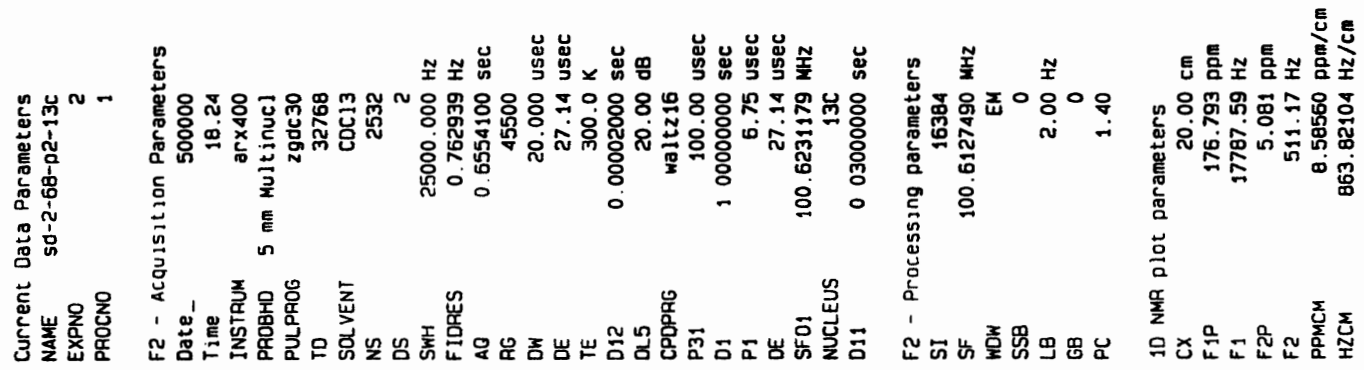



ОВЕ. 29

SमG. $2 L$

$\angle 86^{\circ} \nabla$

$988^{\circ} 9 L$

$101^{\circ} \mathrm{LL}$

$20{ }^{\circ} \angle L$

OZS $L L \perp$

6IE $8 L-$

599. $6 L$

ट0I. 88

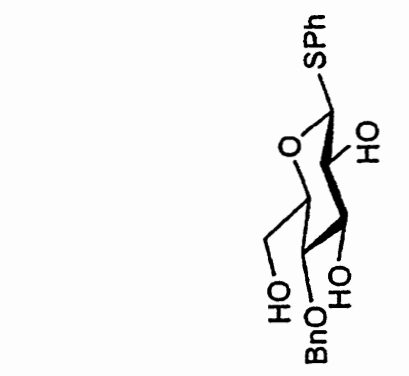

$\left.\begin{array}{l}252.8517 \\ \angle L C \cdot 851\end{array}\right]$

$\varepsilon โ 8^{\circ} 825$

टSE $62 \downarrow$

E98 IET

6I6 टЕ -

दटટ' $8 E$

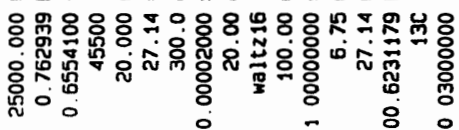

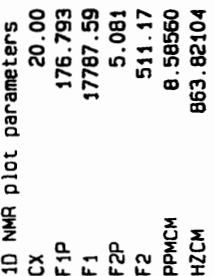



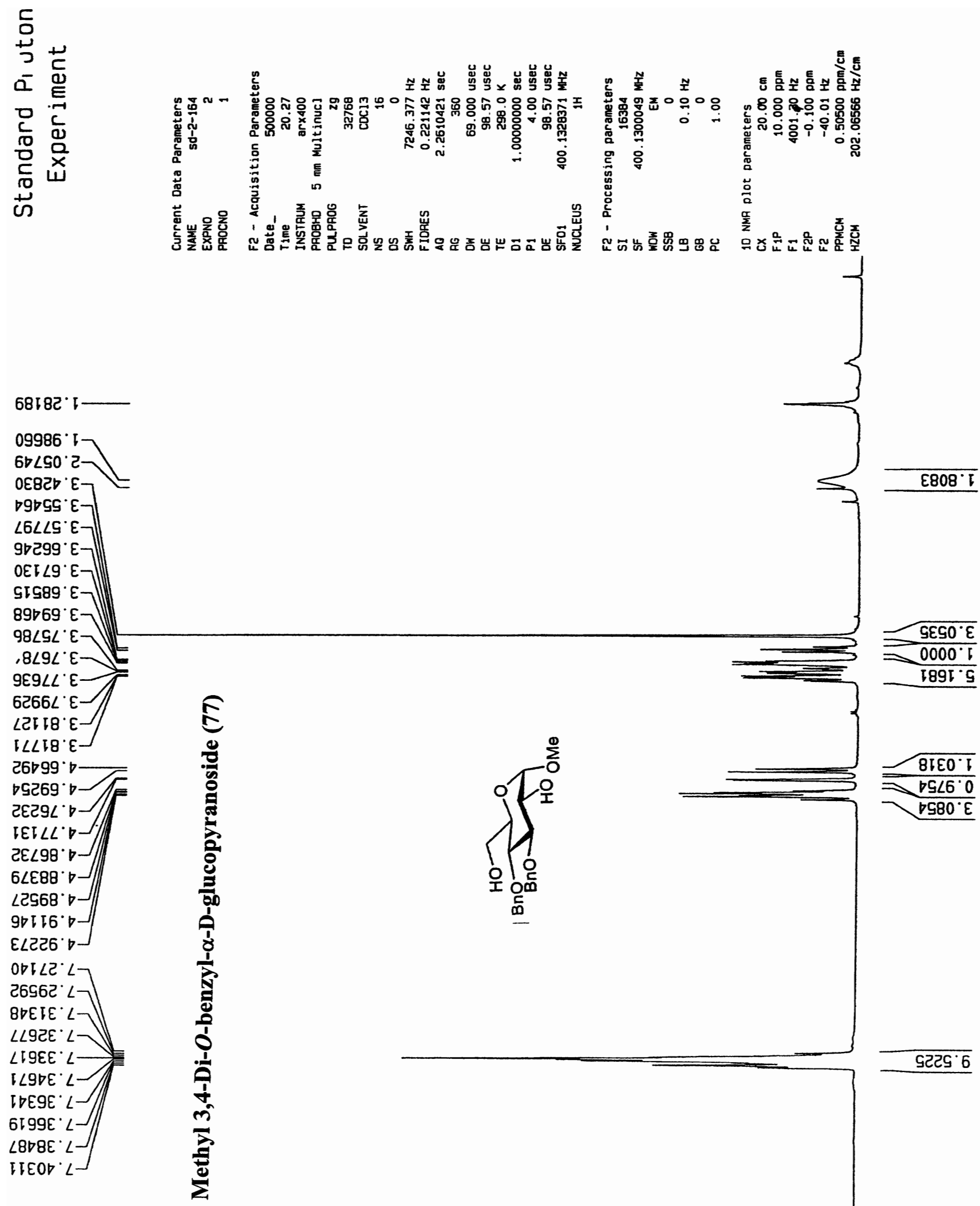

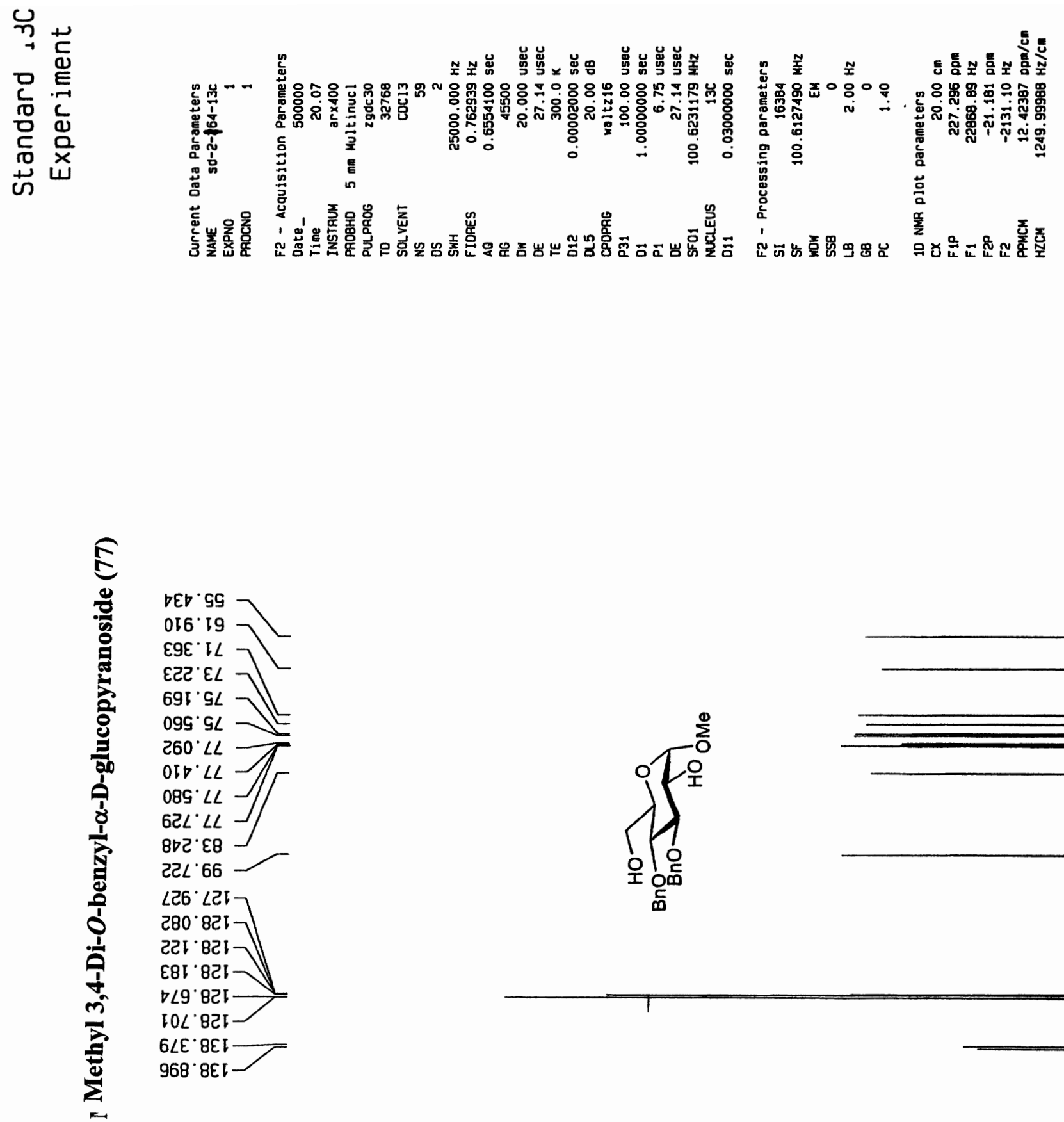


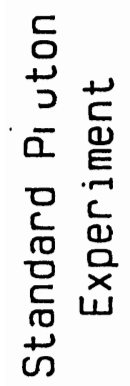

$\angle 2 I \angle 2 \cdot$

โ $682^{\circ} \cdot-$

86 $\angle 6 S^{\circ} \downarrow$

65919.

06॰29.

ดाटЕ9

9DBE9.

टOELI

हट08

09195.

I IOI9. E]

LZZE9' $E-$

6โ6E9 $E$

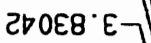

टES98 $E-$

$99 \varepsilon \angle 8^{\prime} \varepsilon-$

$58996^{\circ} E-$

$3 b \angle \varepsilon 9^{\circ} \bullet-[$

$\checkmark 999^{\circ}$

8 ㄴ $89^{\circ}$

IS29L

$610 \angle L D$

SGELL

$66 \angle 8 L^{\circ}$

S9LE '

ES9L6 $\square-$

$0<S 00^{\circ} \mathrm{s}$

9ट9ट2 $\angle$

$6 \angle I E C \cdot \angle$

$\angle S E D C^{\circ} \angle$

टEI $\angle C^{\circ} \angle \longrightarrow$

$\angle \nabla G I E \cdot \angle 7$

ESDटE $L>$

$\angle 692 E^{\circ} \angle$

ЕEОEE ' $\angle$

$\triangle 8 D E E^{\prime} L$

OIGEE ' $\angle$

$\angle$ LDV $\angle$

6EOSE

666SE' $L$

8006E $\angle$

OBE6E $\angle-$

IIESE $L$
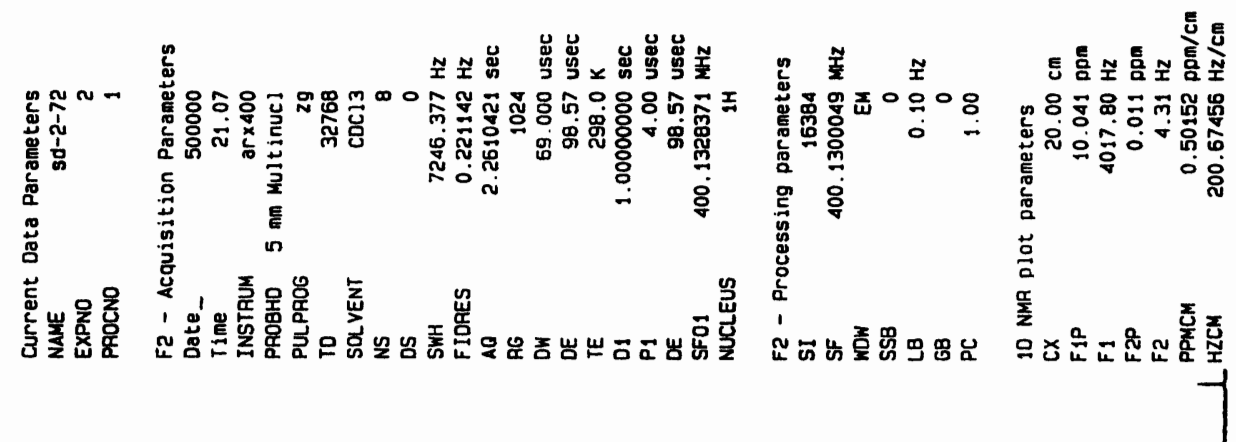


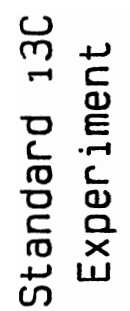

等

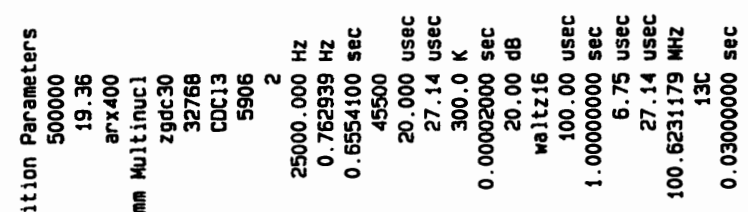

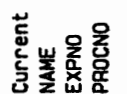

章

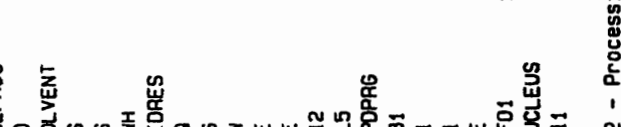

蓄

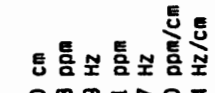

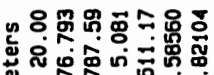

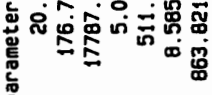

용

$62 L .92$

$006^{\circ} 62$

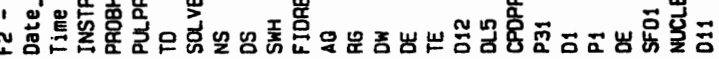

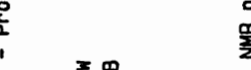

旁
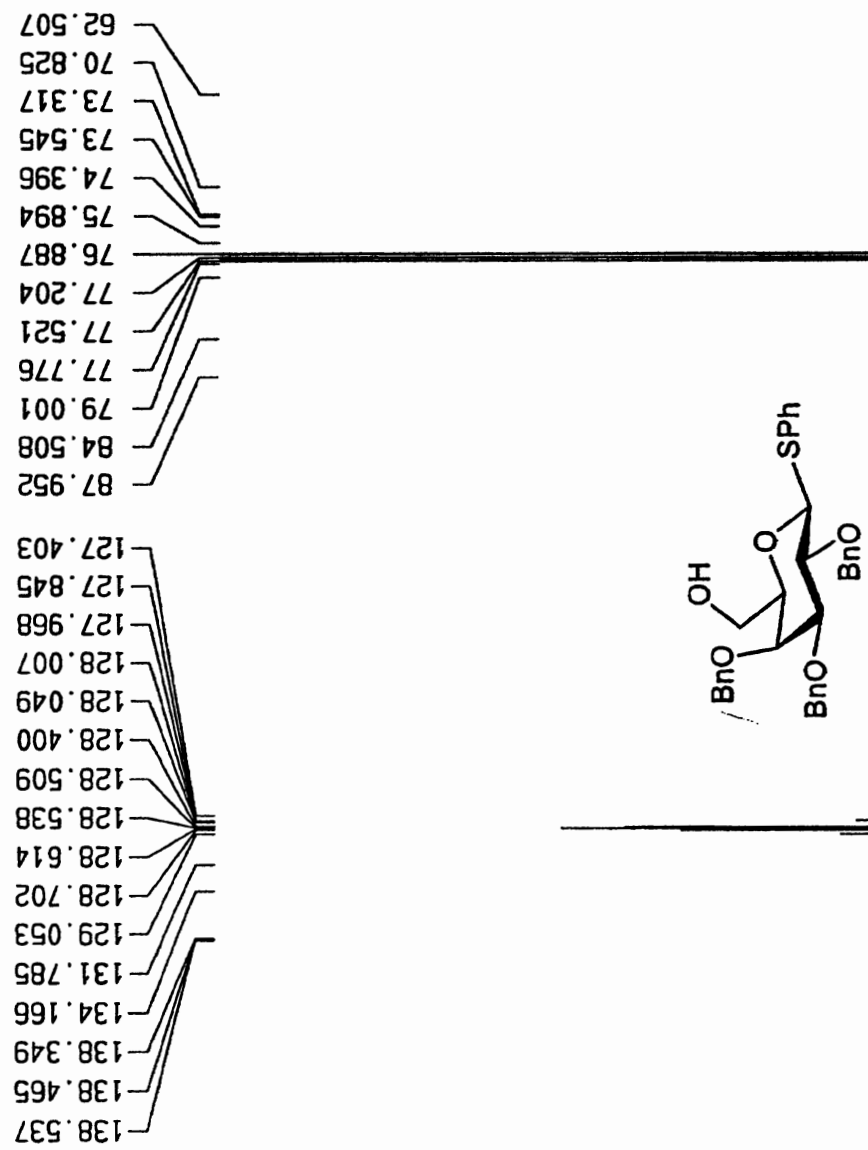

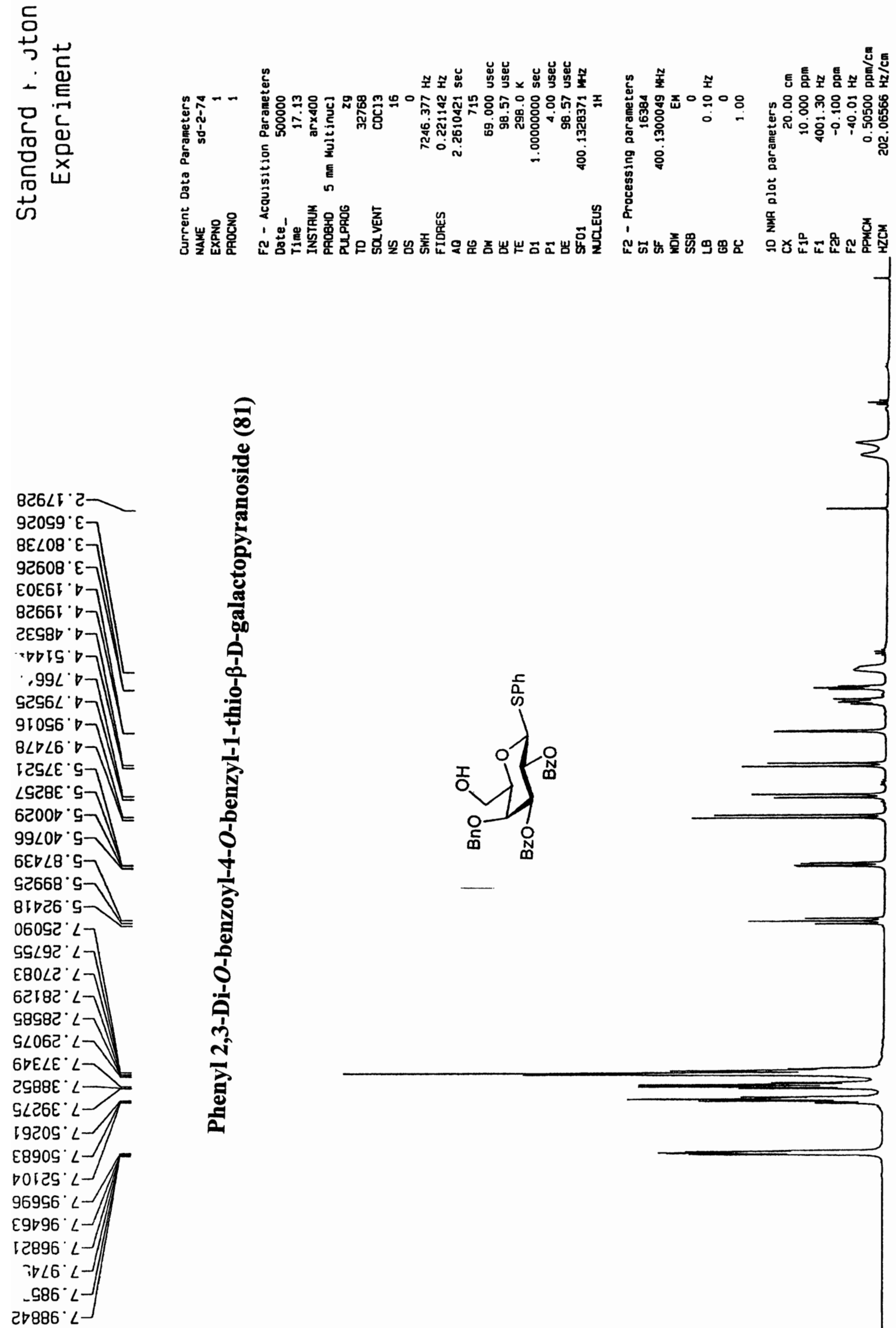

$\frac{\sqrt{4}}{4}$ 


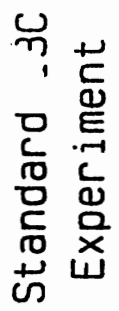
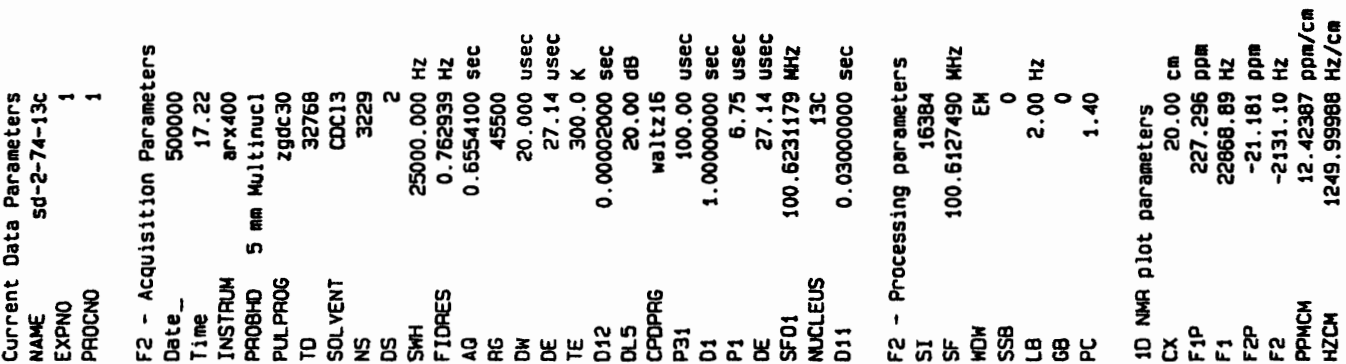

19.
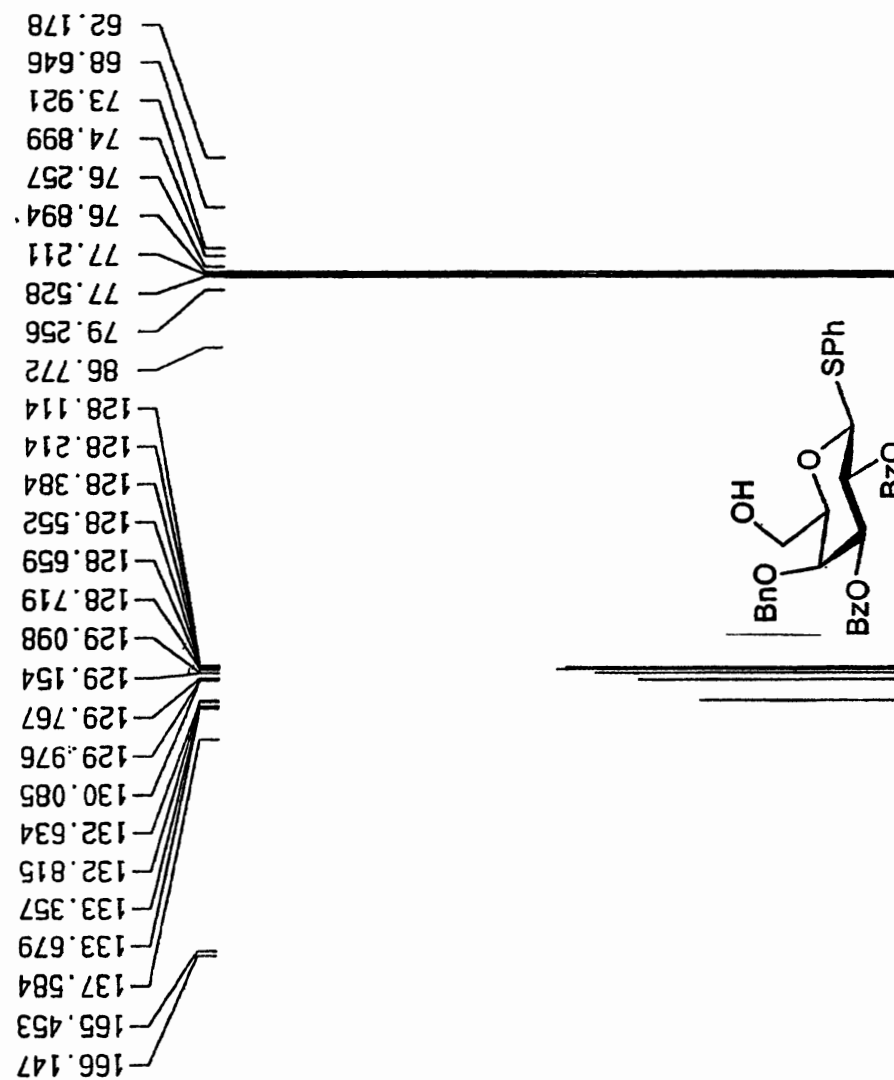


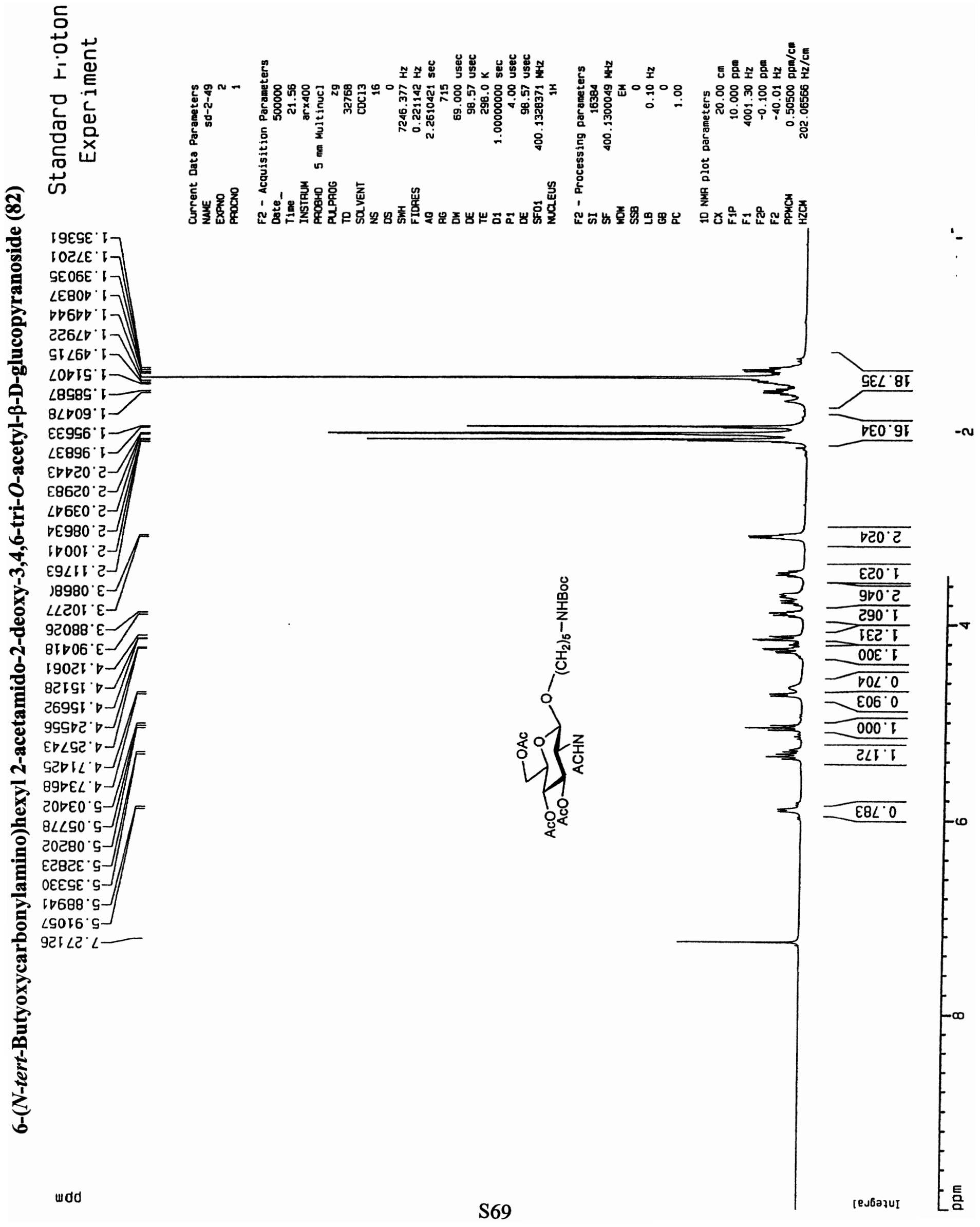




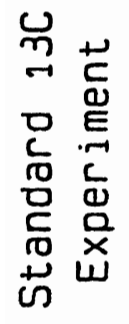

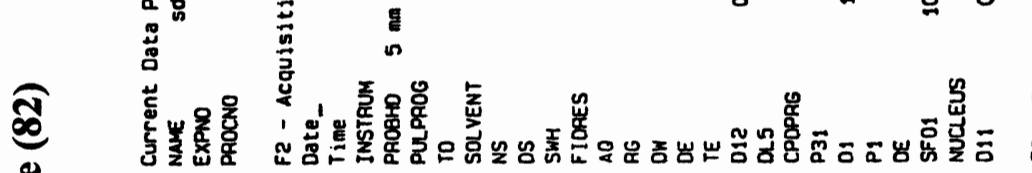

포 똤

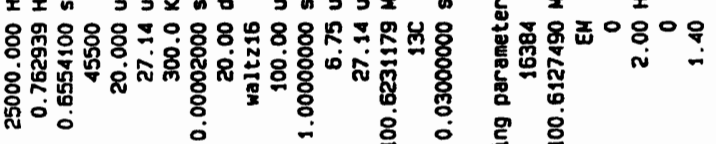
要

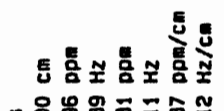

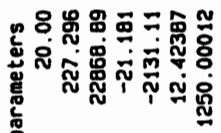
亭

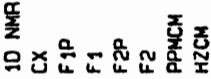



$928.02-$

898.05

DEG.0Z

ट8० $\varepsilon ट$

189.9?

$869^{\circ} 62$

$6 \varepsilon L \cdot O D$

982 ' $\mathrm{SG}$

$66 E^{\circ} \mathrm{ig}$

$098 .<9$

150.69

$\angle$ 18.69

$866^{\circ} \mathrm{KL}$

$\triangle 8 D^{\circ} \mathrm{CL}$

$988^{\circ} 9 L$

DOC $\angle L-$

$\angle 8 E \cdot 6 L]$

$0 \angle 8^{\circ} 00 \mathrm{~K}-$

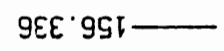
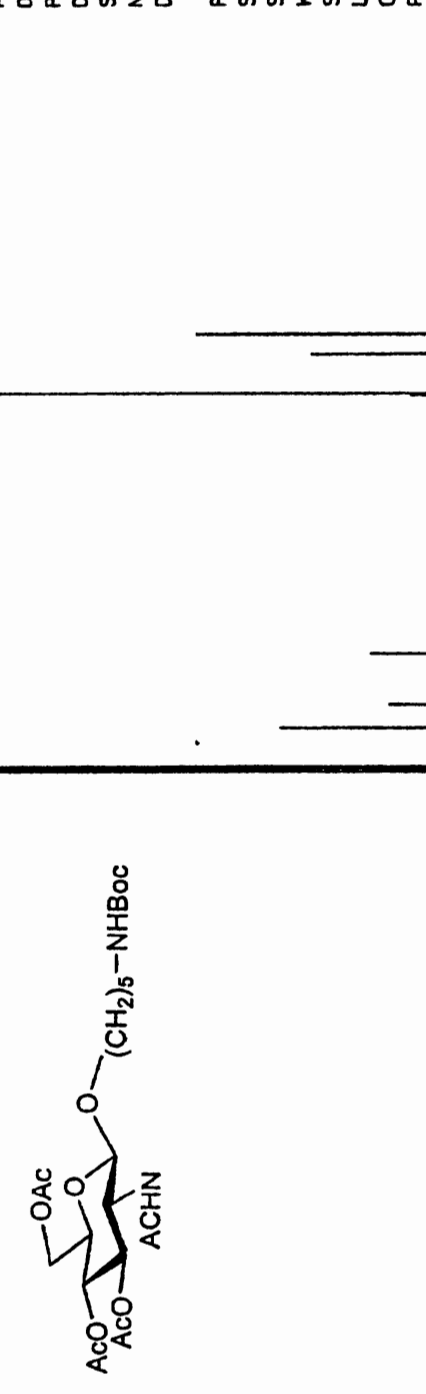

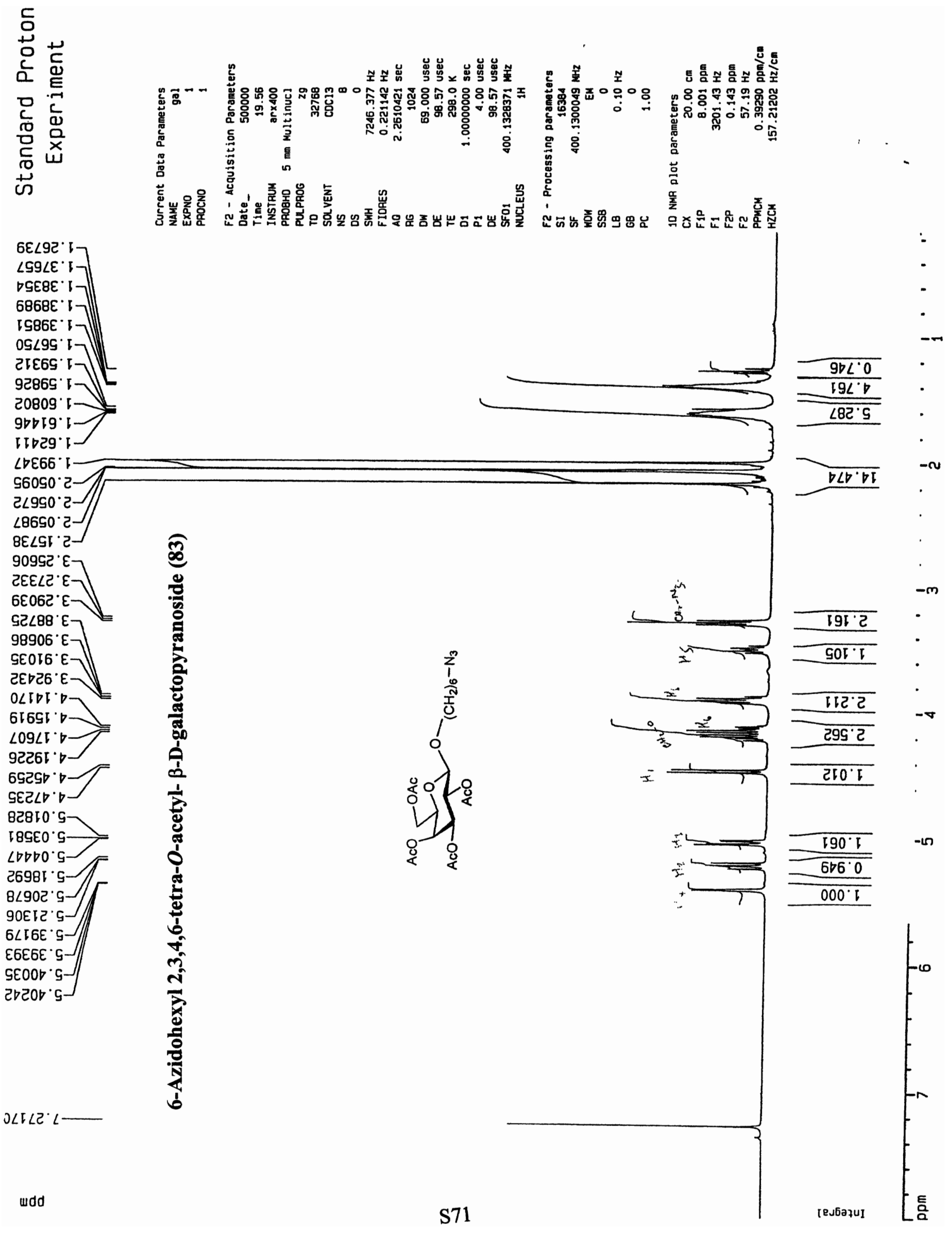


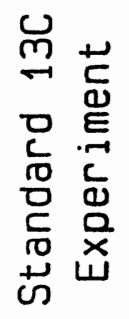

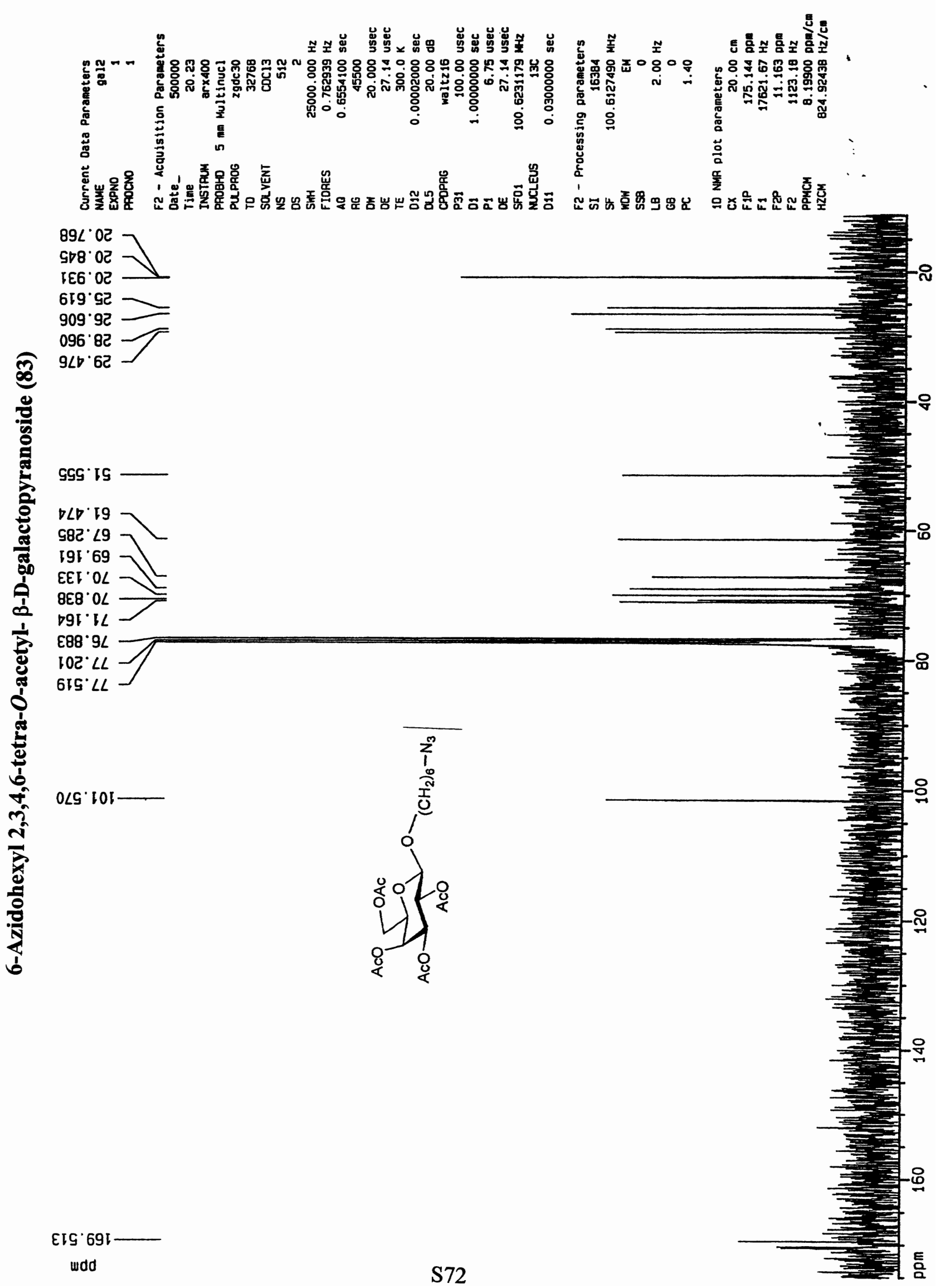




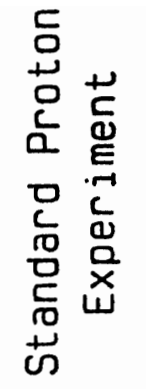

Eเ66! 1 $\square \angle D L C^{\circ}-7$ ट980ट. SOSLC' $E \angle S 82$ ' [$\angle E S L E$ ' $\triangle 826 E^{\circ}$ BOEET : Dट996' I $69980^{\circ} \cdot-$ ¿ $\angle O S^{\circ}$. I IEZS I96889 $\leftarrow$ $\angle \nabla 809^{\circ} \mathrm{L}-\mathrm{C}$ $\angle 92 \angle 6^{\circ}{ }^{\circ}-$ $80 \angle E 0^{\circ} \mathrm{C}-$ 9IEEI' $2-$ S9LDI' 2$]$ $\angle G D O I^{\circ} \varepsilon-$ 6EOटा $E-$ $\checkmark 8 \nabla 2 \nabla^{\circ} \varepsilon-$ $\varepsilon \varepsilon 9 \nabla 9 \cdot \varepsilon-$ $\angle E O \angle 9^{\circ} E-$ $62969^{\circ} \square$ $\mathrm{G} 6000^{\circ} \mathrm{G}$ $29690^{\circ} \mathrm{G}$ 8286 I $^{\prime} \mathrm{S}$ $8 \checkmark 202 \cdot 9$ $6 \angle 902^{\circ} \mathrm{s}$ DOTIS. 29852 $9-$ I $\angle 92 \cdot \mathrm{S}-$ SLE82 9 sटट62's-

IE692 $L$
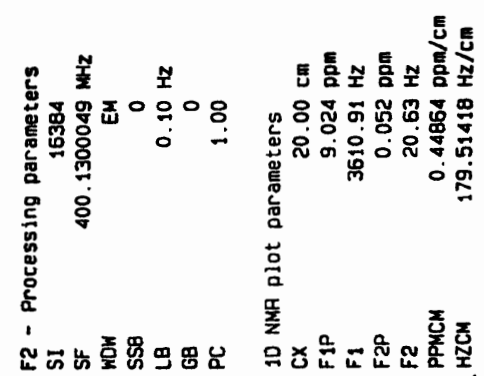

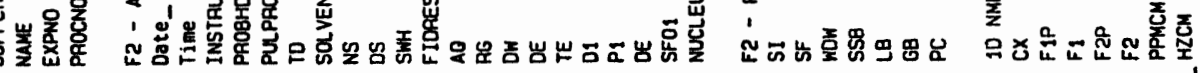
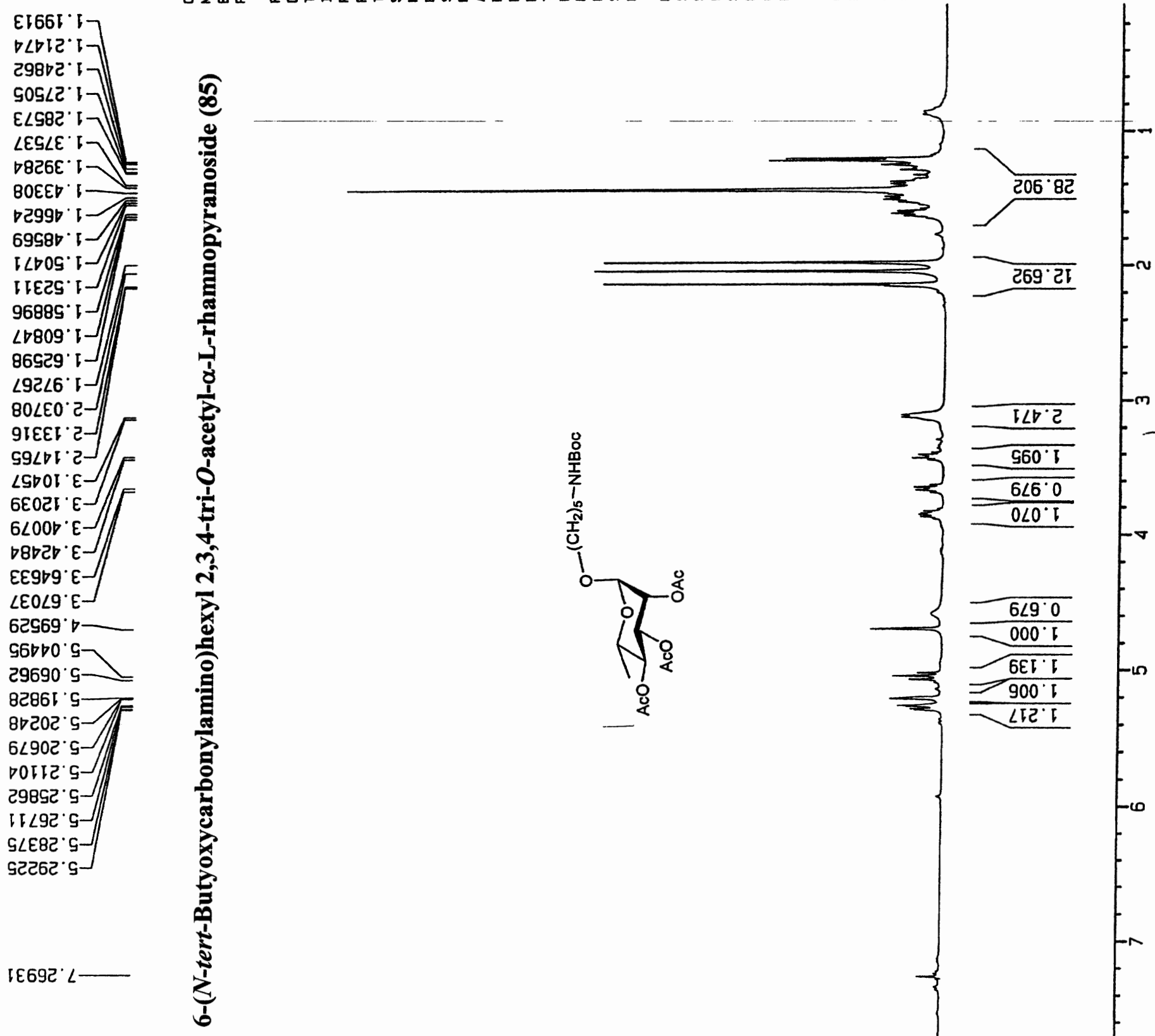


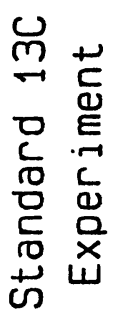
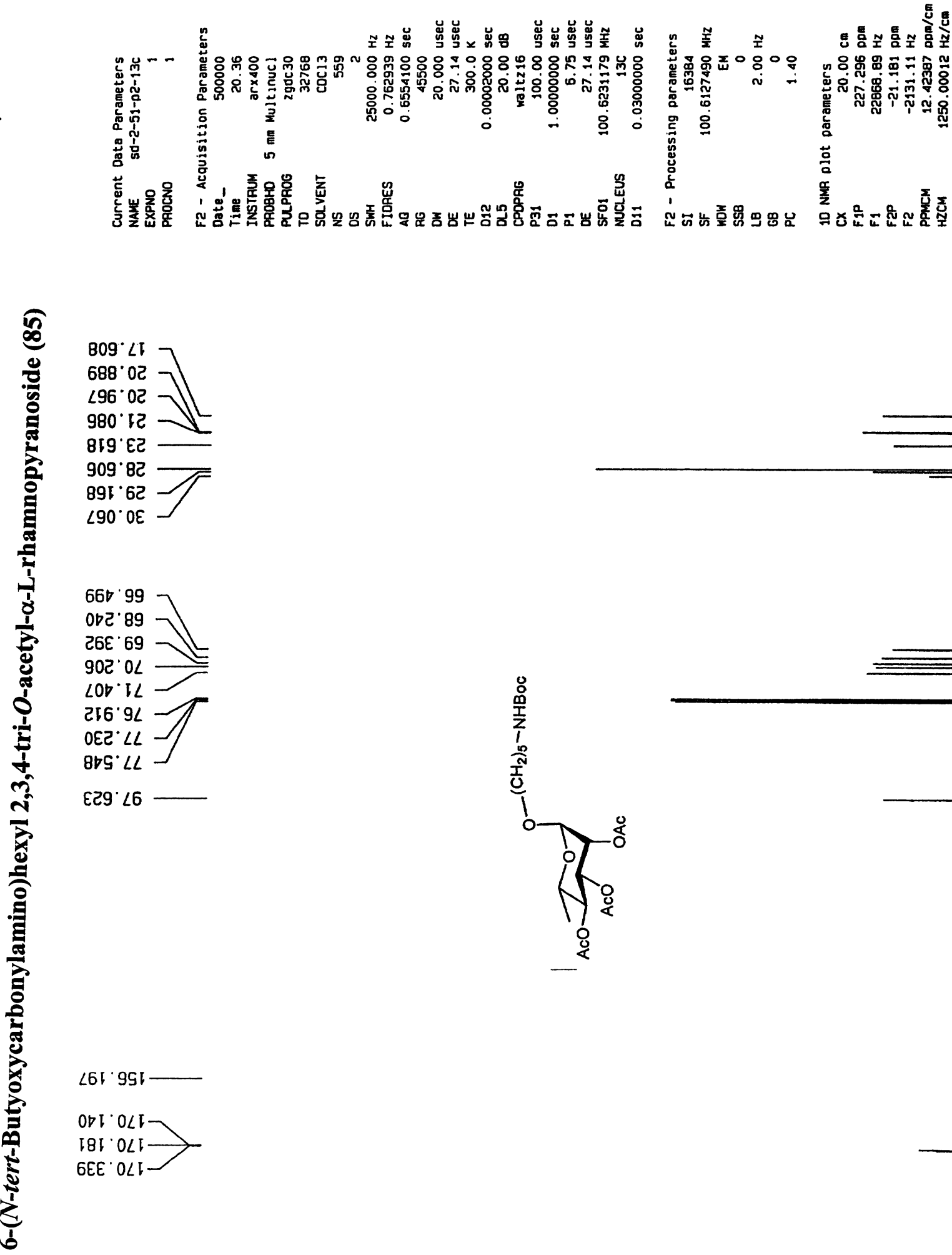


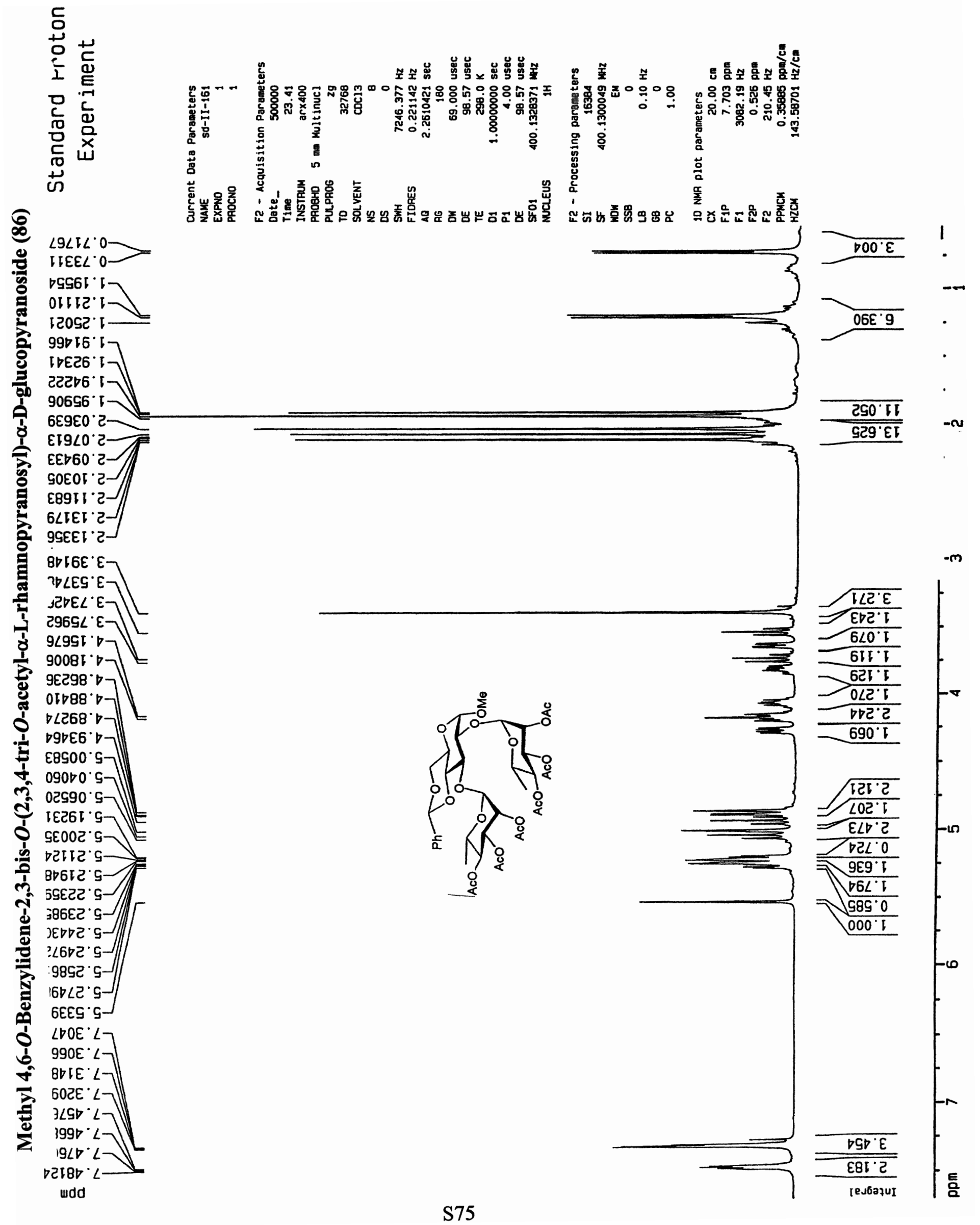




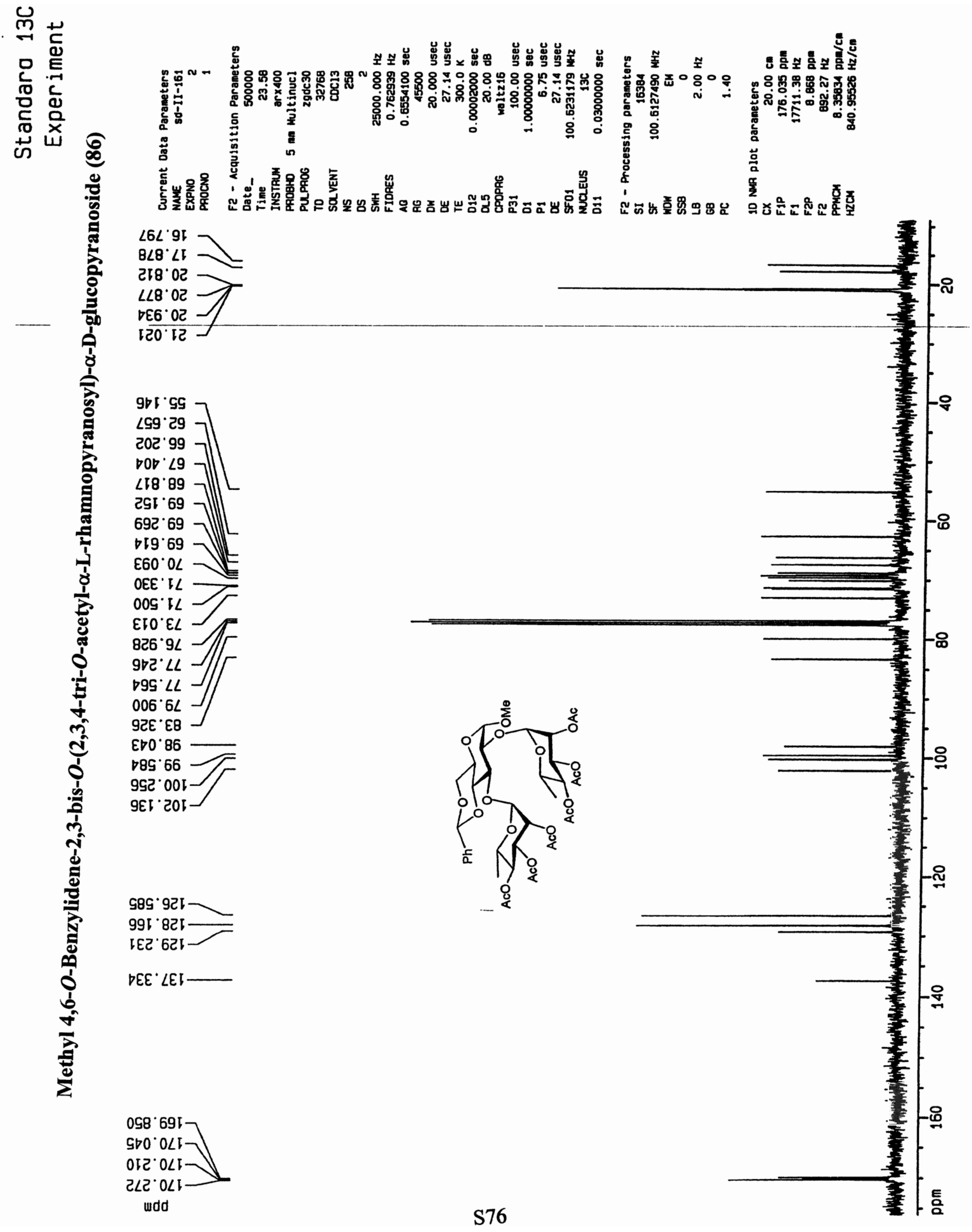



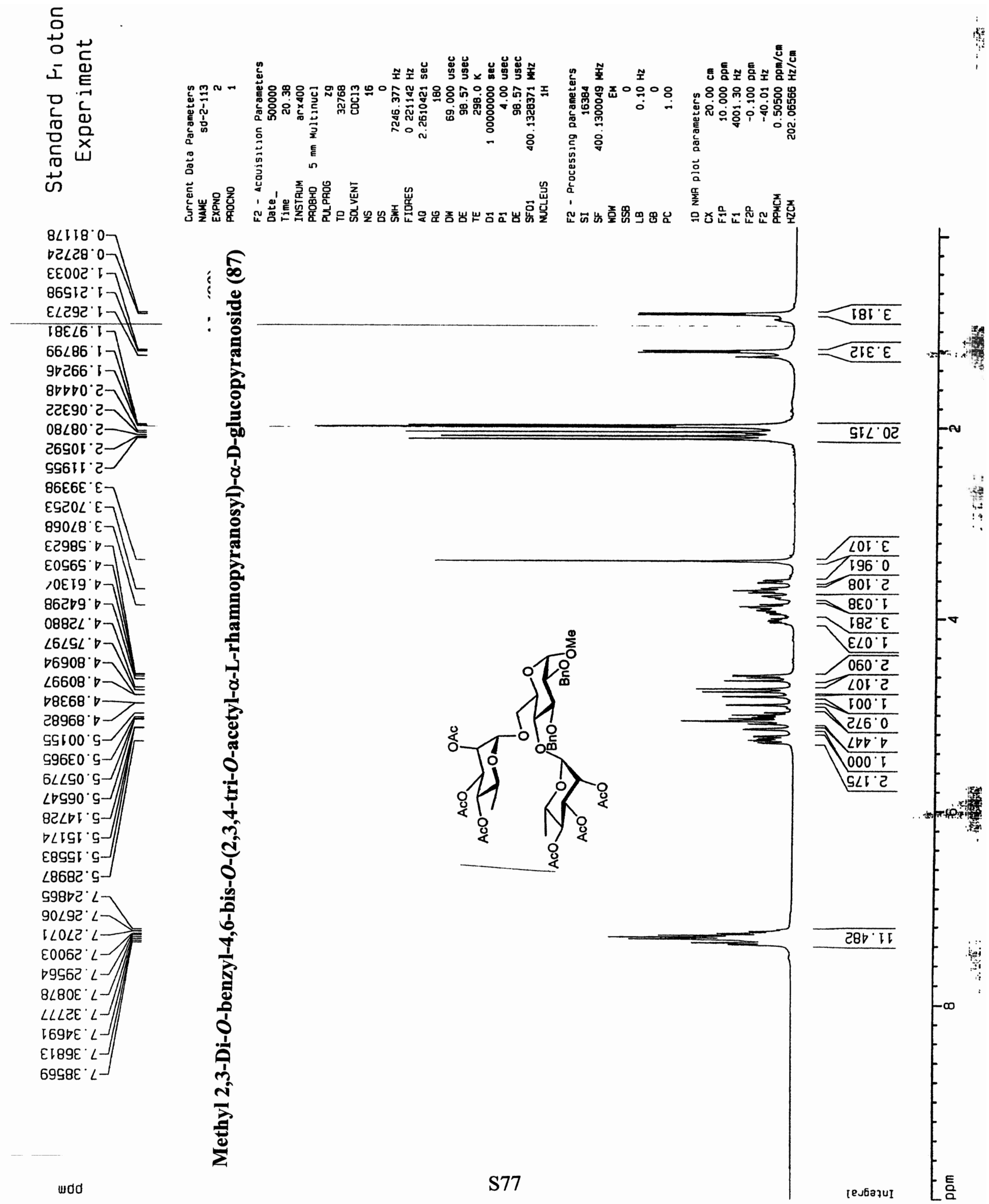


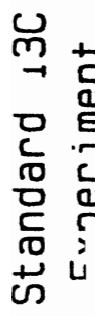
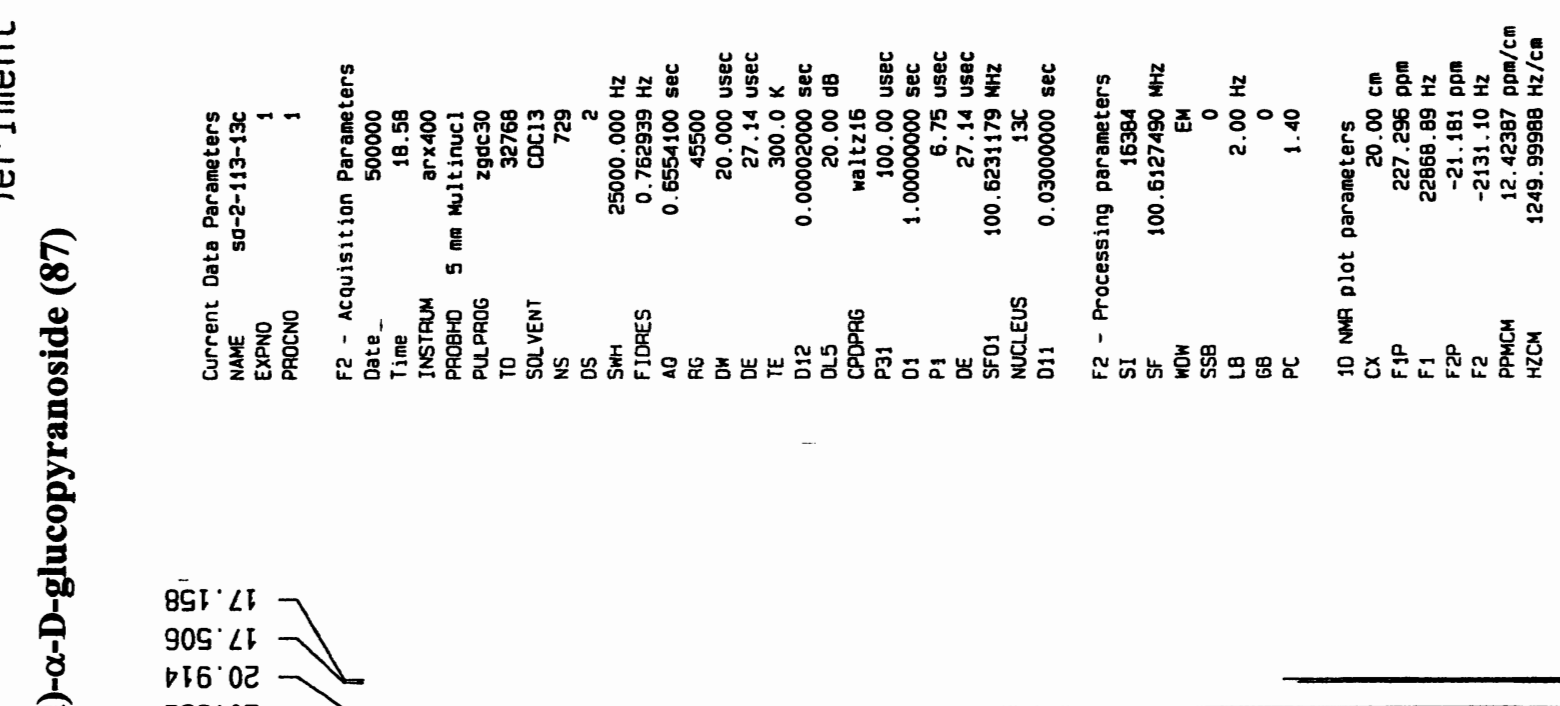

$286^{\circ} 02 \bigcirc$

Eटम'SG

$65 \overline{6} \cdot 99$

E9l' $\angle 9$

ร9l 69

टEट'69]

$\triangle 89.69$

966.69

SEE' 02

DOI' $L L$

$\varepsilon \angle \mathcal{S}^{\prime} \mathrm{V} L$

$G \angle G^{\cdot} E L$

I8S' 9

IEG ' $\mathrm{S} L$

$800^{\circ} \angle L$

$\angle 2 E^{\prime} \angle L$

SD9. LL

$\triangle 2 I \cdot 08$

I $\angle 8^{\circ} 08$

I $\angle 9^{\circ} \angle 6$

$6 \nabla 8 \cdot \angle 6$

$856 \circ 6$

$180 \cdot \angle 25-$

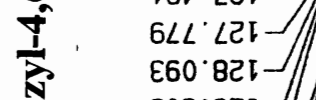

EOE. 851

ồ

टा9.8टा

Gटट $8 E 1-$

$\triangle 16.8 E 5$

076.691

266.691

$\angle 9 I^{\circ} 0 \angle I-$

CLC $0 \angle I-$

E89 $0<\mathrm{I}$ 


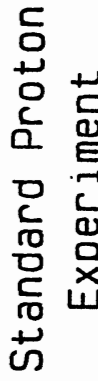
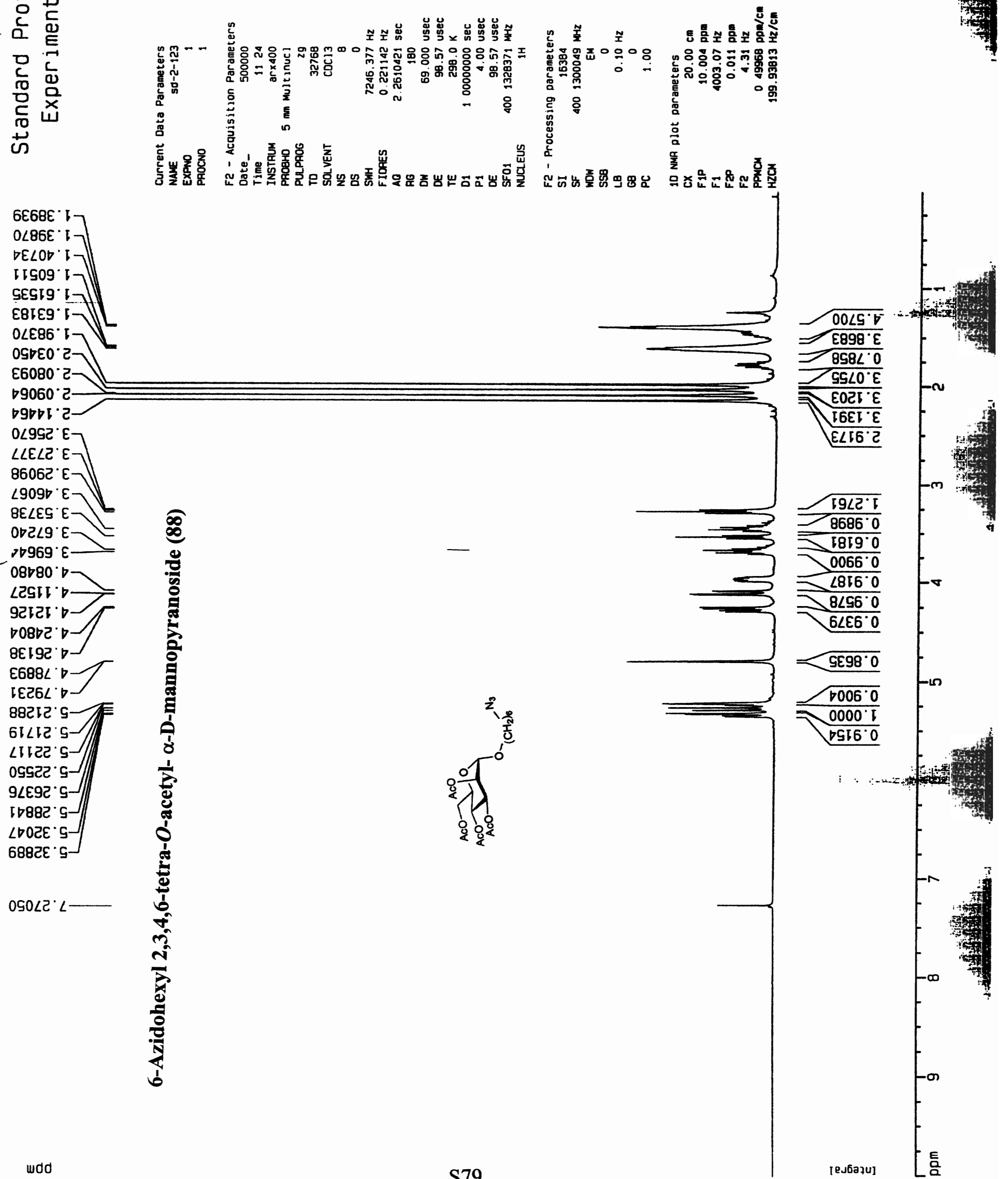


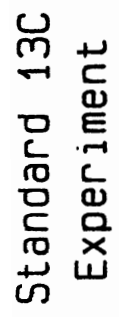
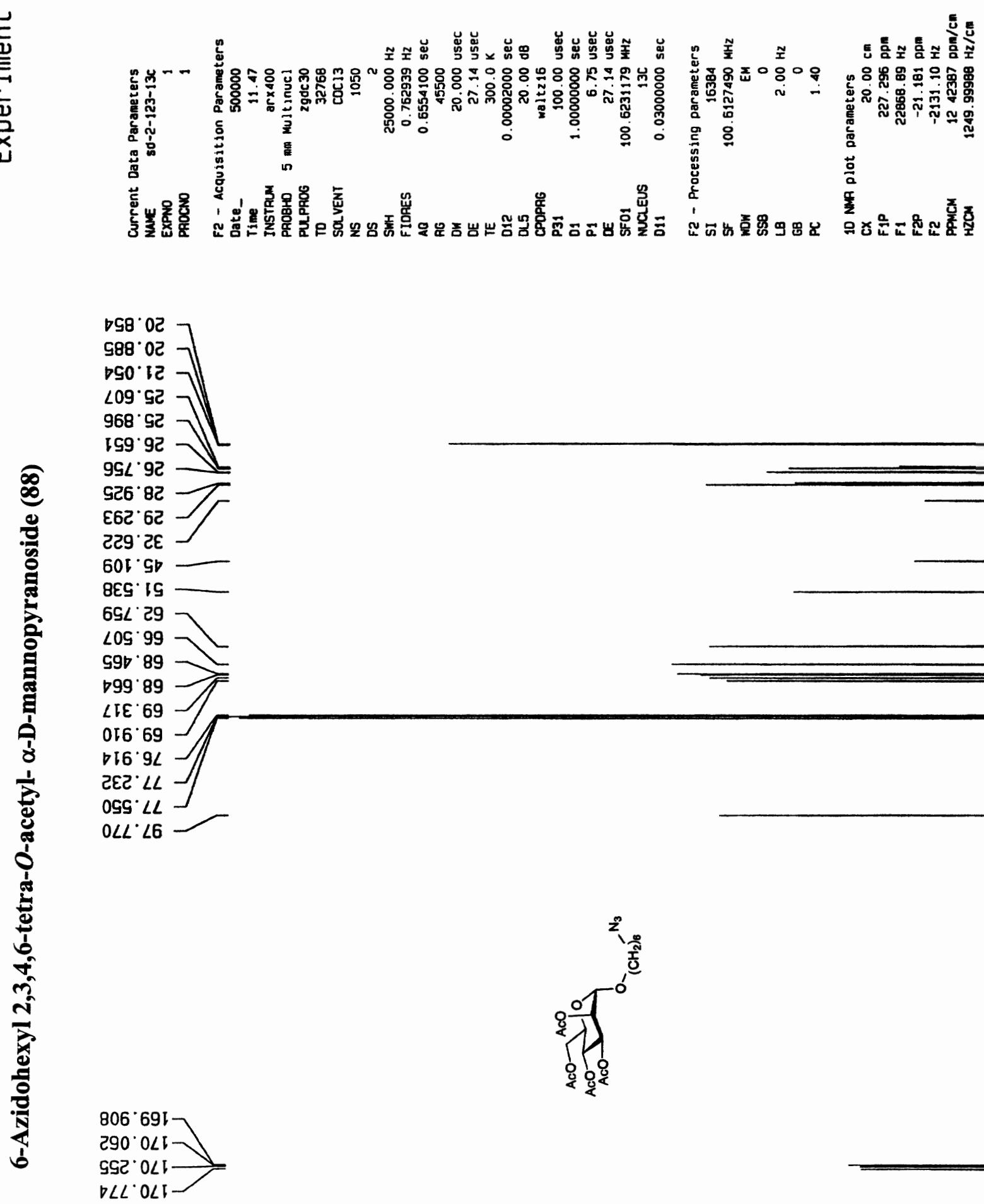


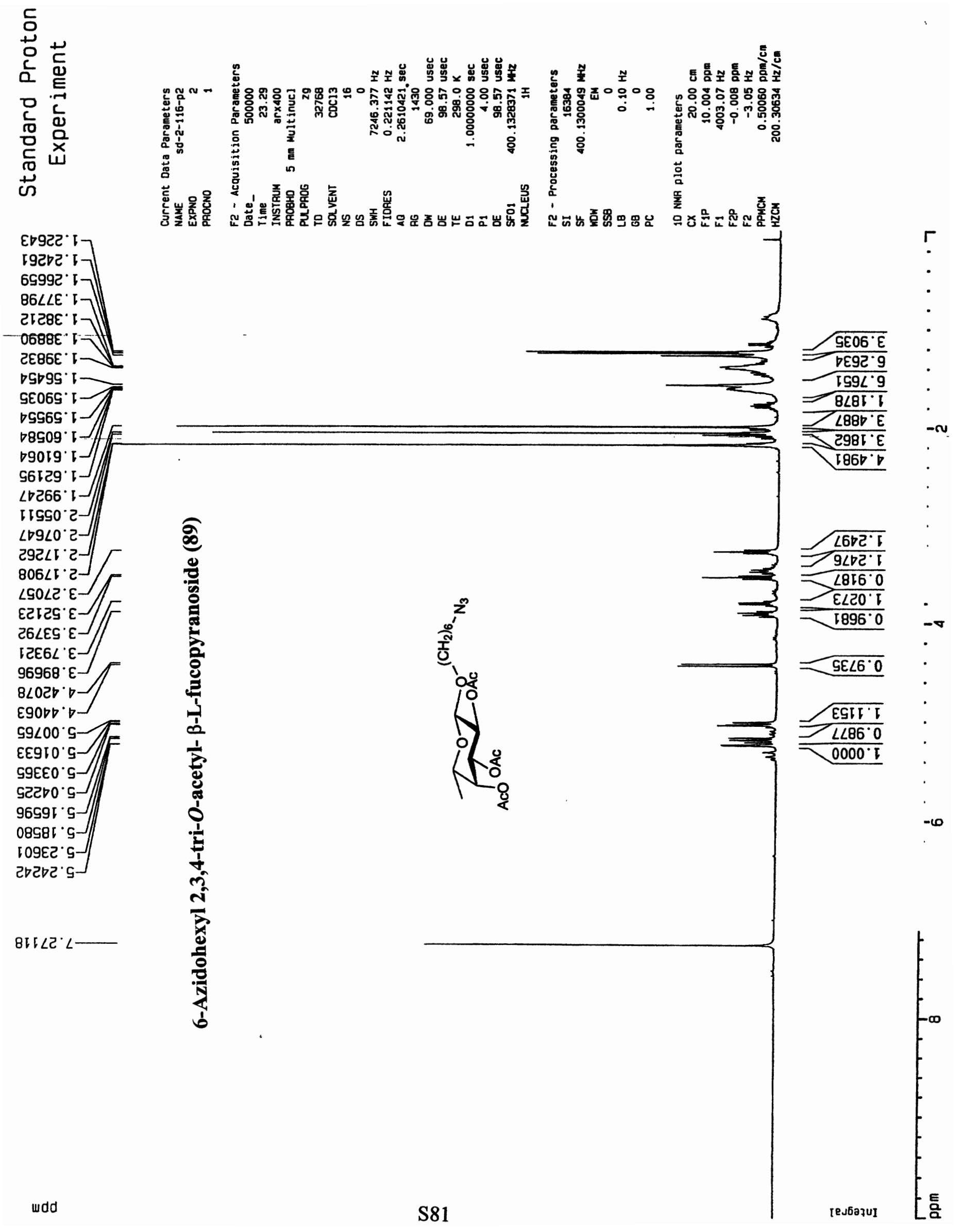



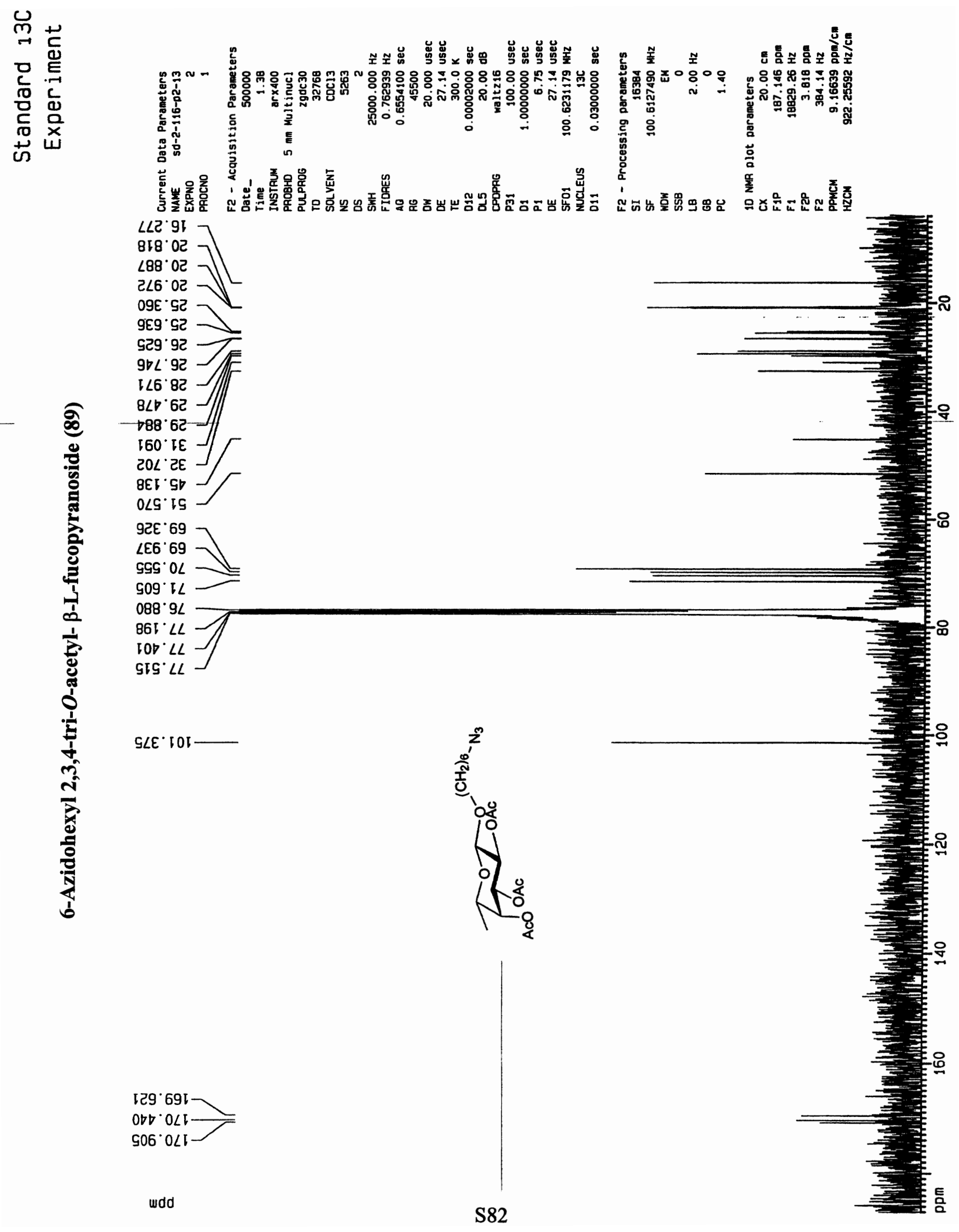

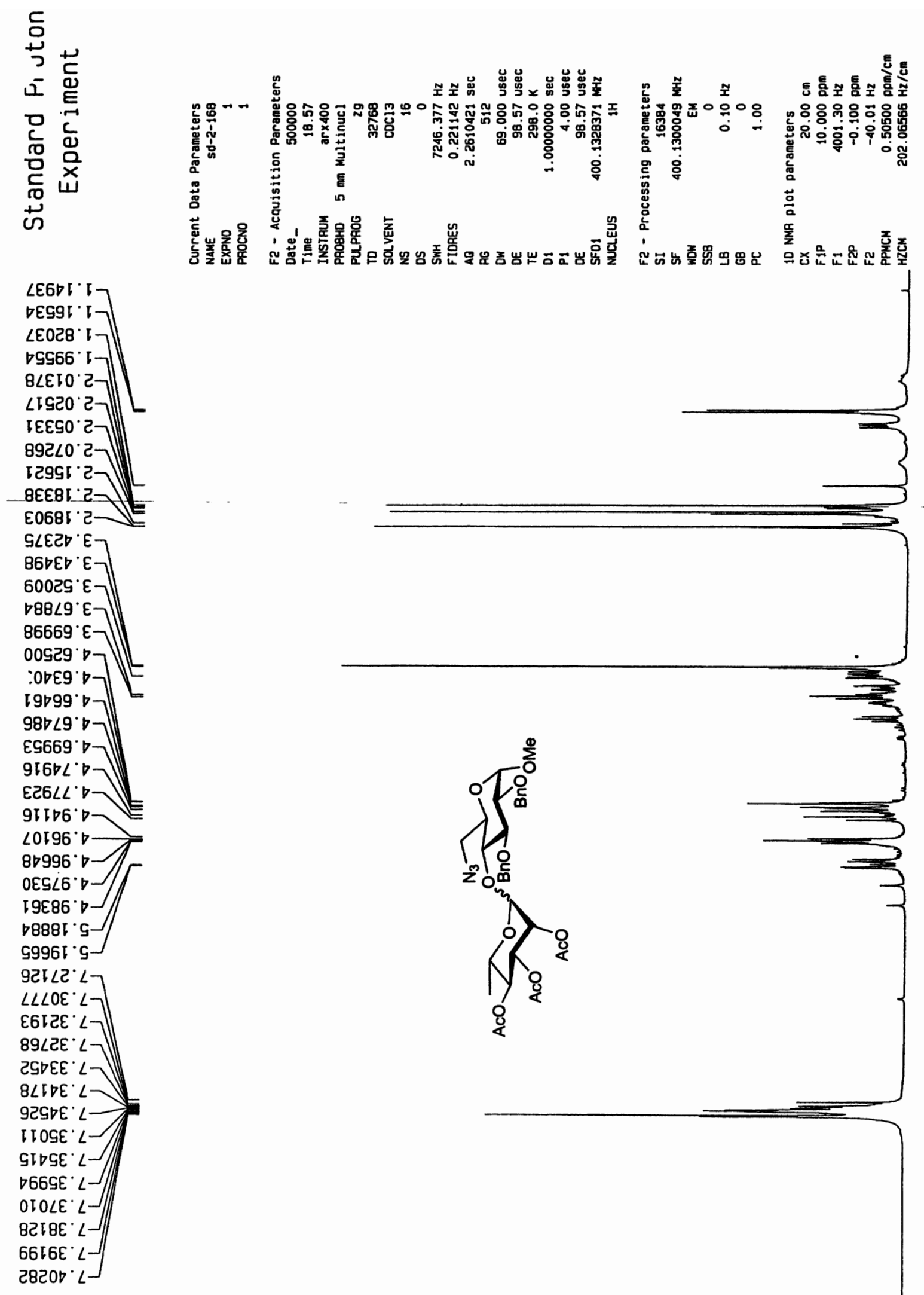


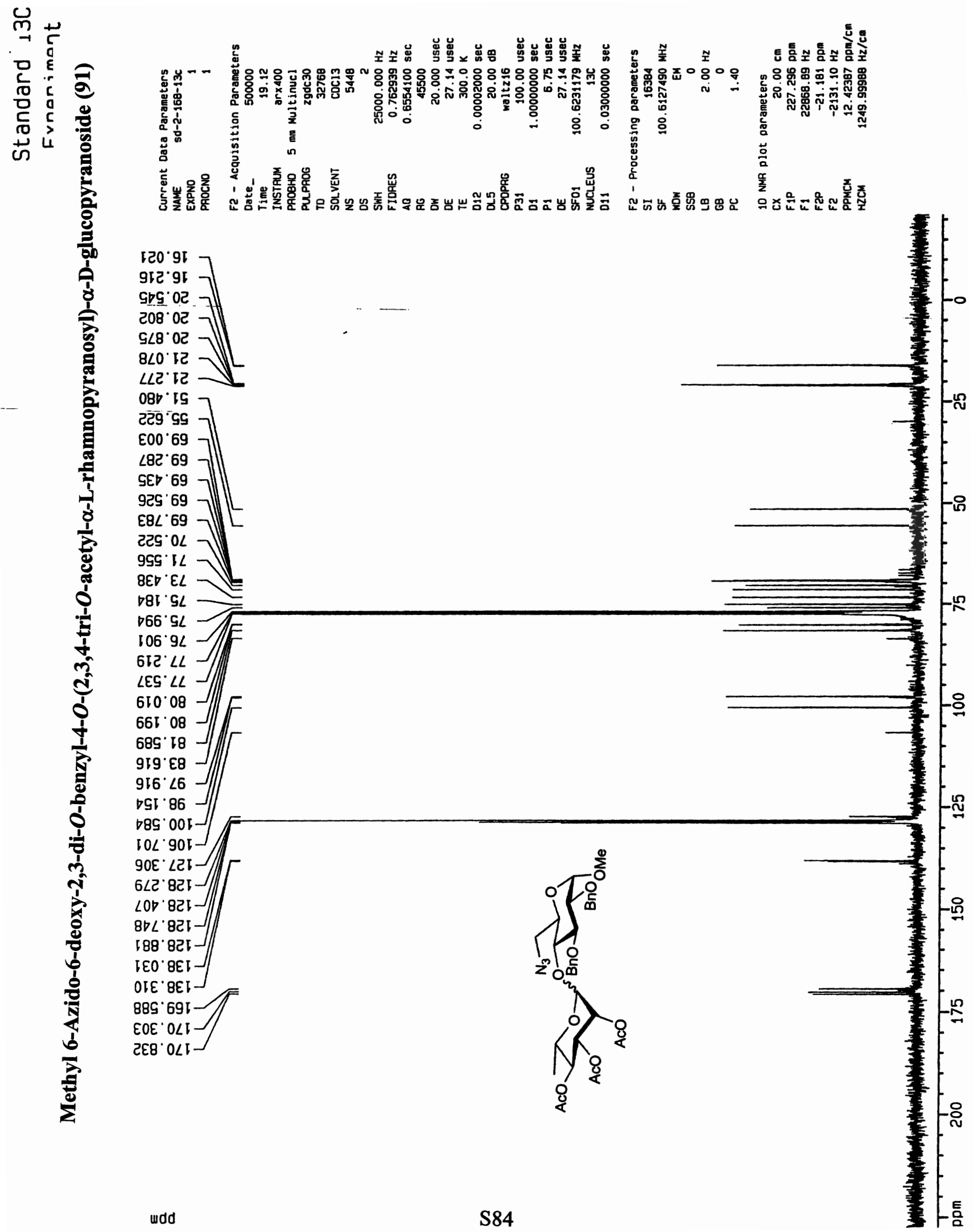


동

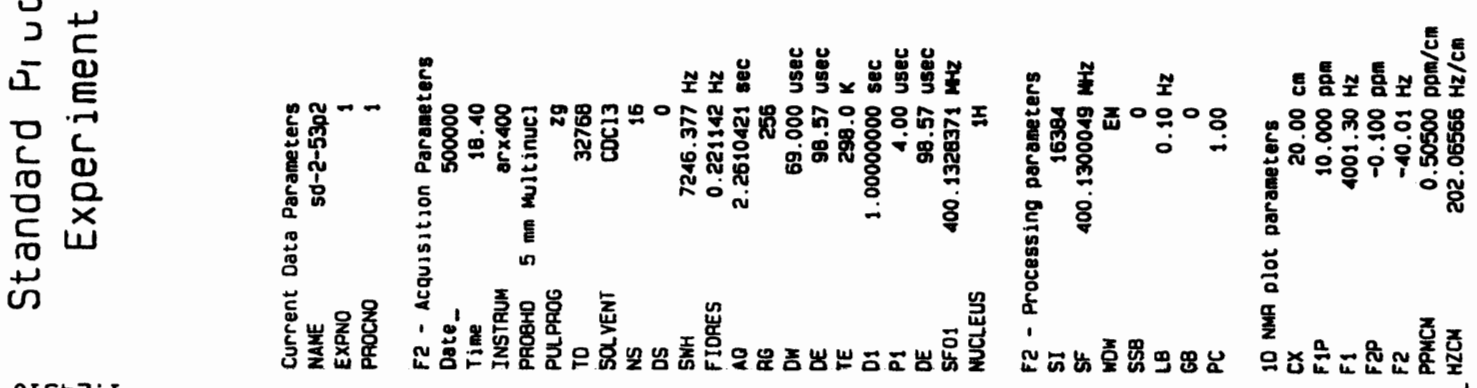

OL6D2 '

SDO6E. [-

टD०6E' [-

BS[LO'

$\angle \angle 6 I V$

BLEED $\cdot 1$

DLEZS

SBIVG

EIBSG

$\triangle S \angle L S$.

$80665^{\circ} \mathrm{I}$

$\angle E 100^{\circ} \mathrm{C}$

$1 \angle L 20^{\circ} 5$

OIOEO 2

$0 \triangle \square \angle 0^{\circ} \mathrm{C}-$

$8 E 6 \angle I^{\circ} E$

OSD6I $\varepsilon$

$59602 \cdot \varepsilon-$

. $0919^{\circ} \varepsilon$

ОSटE9 $\varepsilon$

Eะ8७9 $\varepsilon$

9ESटा $\square$

$\angle D O E I$

$\varepsilon \angle 6 \angle 2 \cdot \square$

$09062 \cdot \nabla-$

B60IE' $\square$

$89890^{\circ} \mathrm{S}$

$59980^{\circ} \mathrm{s}-$

$\checkmark 2680^{\circ} \mathrm{G}-$

9DIII $\mathrm{G}$

OBDEI 9

$08822^{\circ} \mathrm{s}$

LEटSE '

จ6เo9.

$08299 \cdot 9$
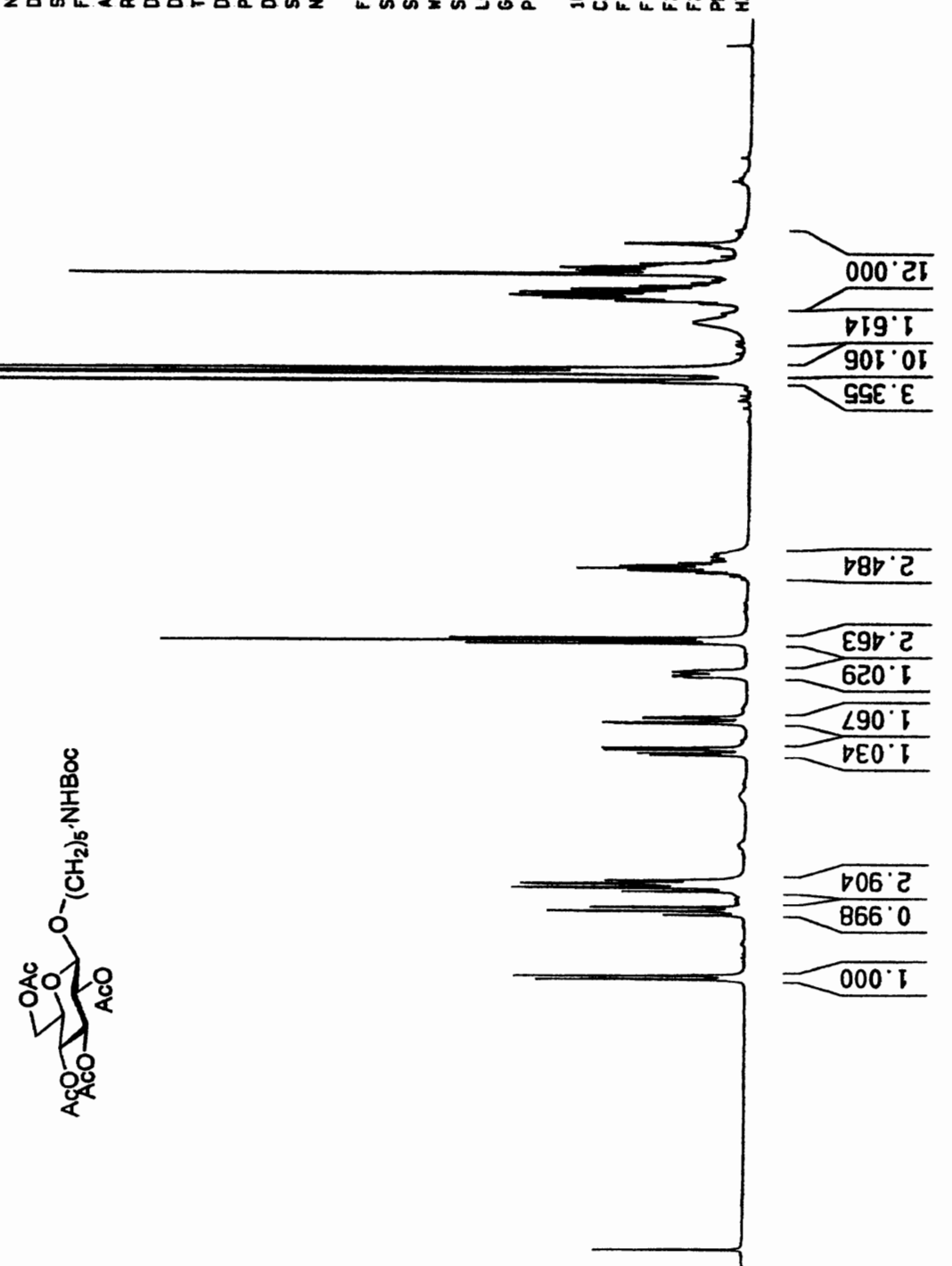

DOLLC L -

$880^{\circ} 2$

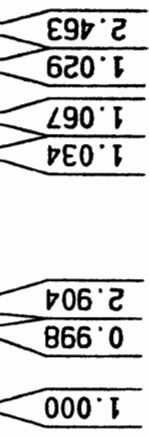




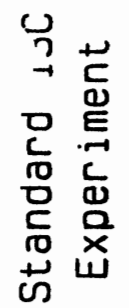
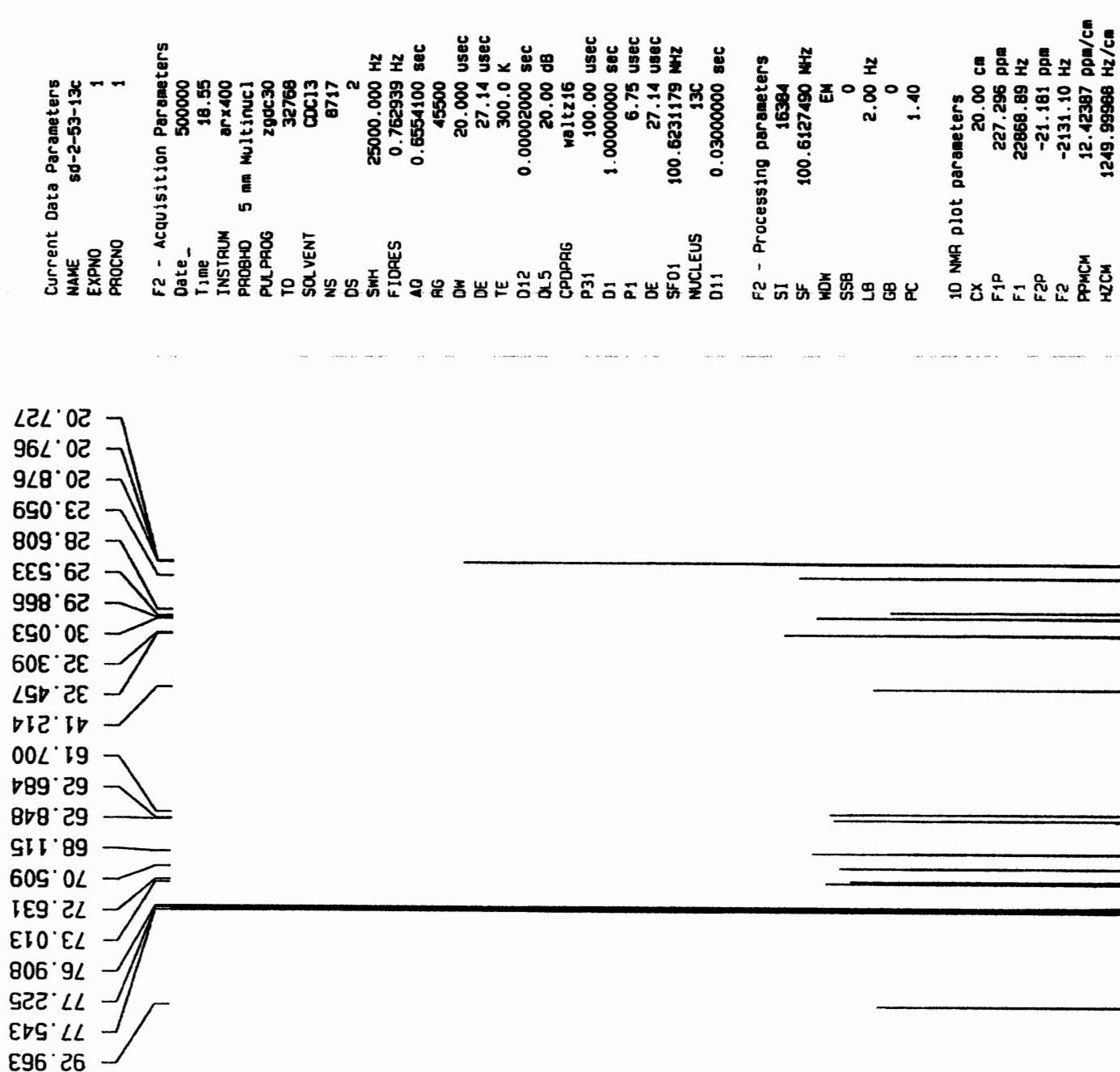

6E6 ES -

SE9. 691

IV $\angle \cdot 691$

हटट $0 \angle L$

Eट8 $0 \angle \mathrm{I}$

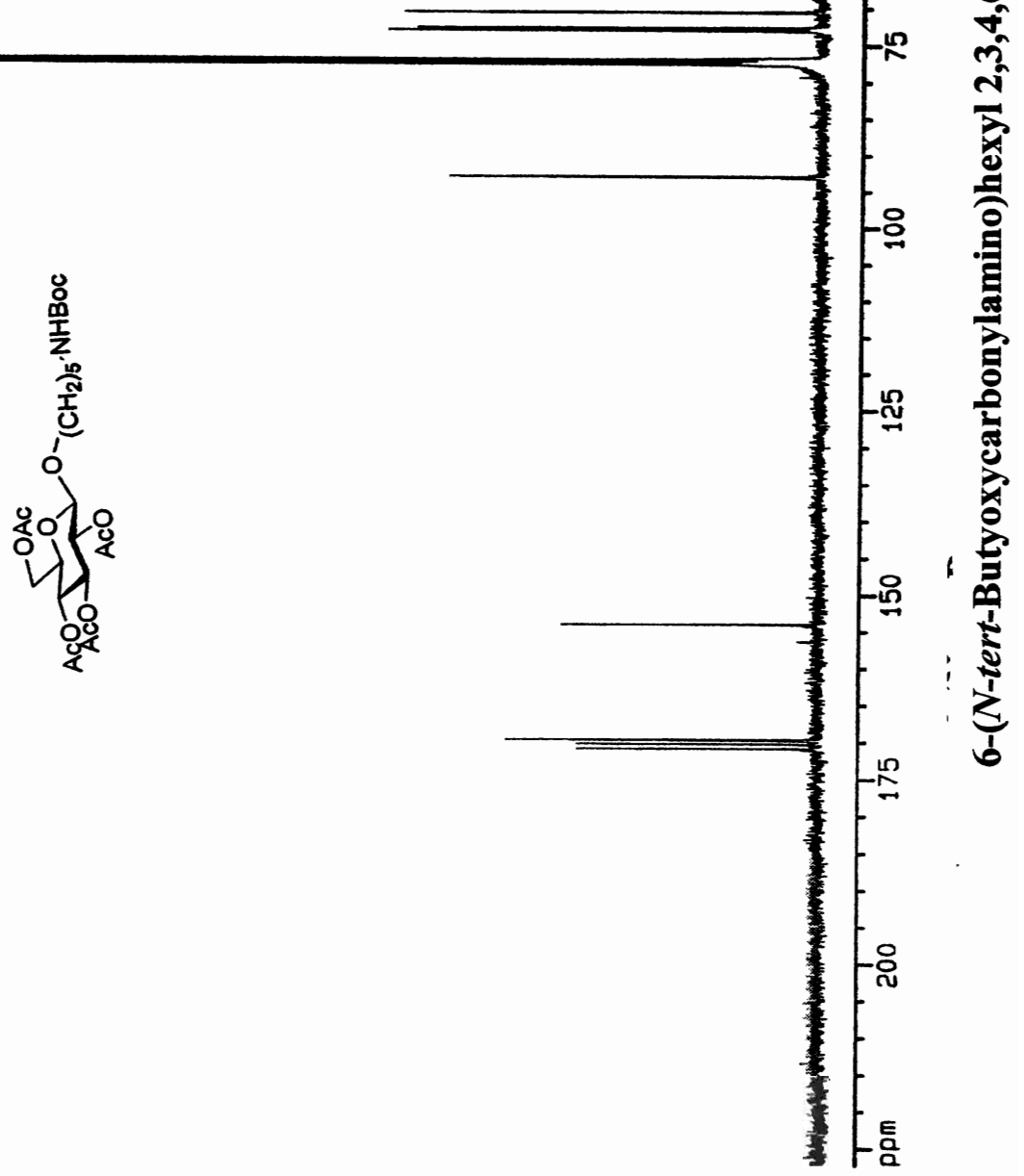




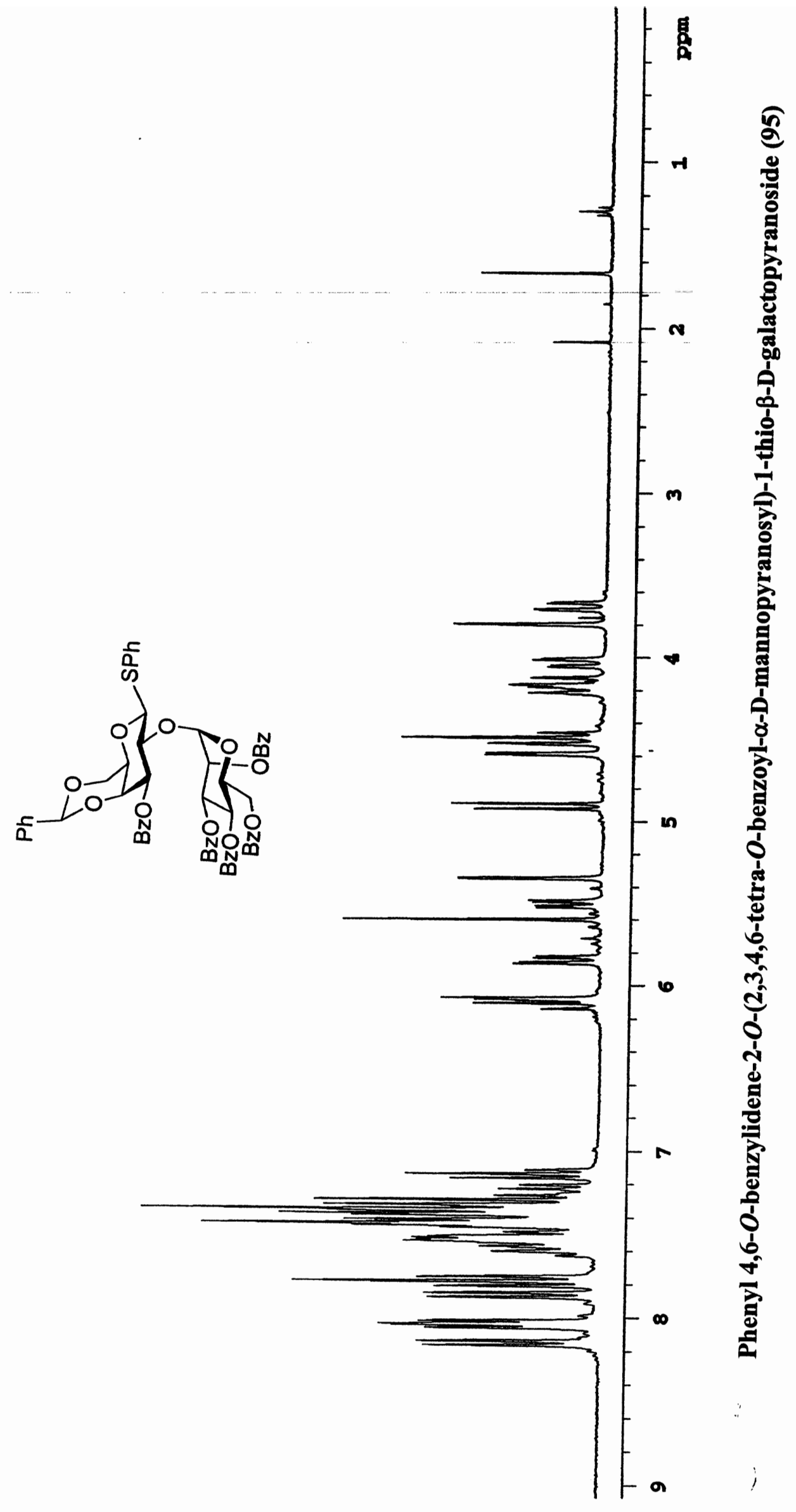




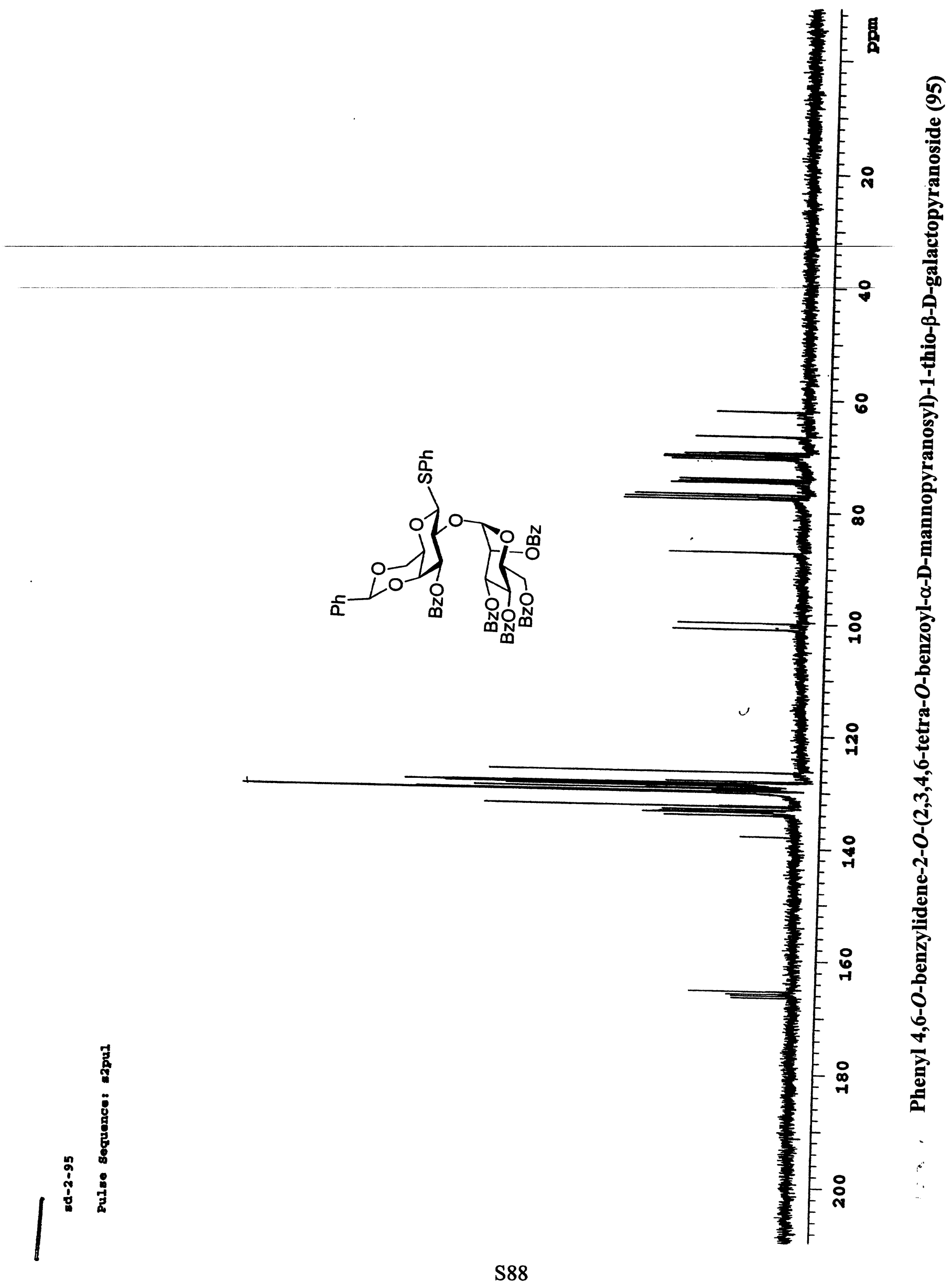




\section{References}

1. Kobayashi, Y.; Ogra, Y.; Ishiwata, Kazuya; T., Hiromitsu; A., Norio; S., Kazuo T. PNAS, 2002, 99, 15932-15936.

2. Commercially available.

3. Hanessian, S.; Lavallee, P. J. Antibiot. 1972, 25, 683-684.

4. Yonehara, K.; Hashizume, T.; Ohe, K.; Uemura, S. Tetrahedron: Asymmetry 1999, 10, 4029-4035.

5. Hui, Y.; Chang, C.-W. T. Org. Lett. 2002, 4, 2245-2248.

6. Evans, M. E. Carbohydr. Res. 1972, 21, 473-475.

7. Szarek, W. A.; Jones, J. K. N. Can. J. Chem. 1965, 43, 2345-2356.

8. Elchert, B.; Li, J.; Wang, J.; Hui, Y.; Rai, R.; Ptak, R.; Ward, P.; Takemoto, J. Y.; Bensaci, M.; Chang, C.-W. T. J. Org. Chem. 2004, 69, 1513-1523.

9. Gilbertson, S. R.; Chang, C.-W. T. J. Org. Chem. 1995, 60, 6226-6228.

10. Deng, S.; Chang, C.-W. T. Synlett. 2006, 756-760.

11. Wang, J.; Chang, C.-W. T. in Carbohydrate Drug Design, Anatole A. Klyosov, Zbigniew J. Witczak, and David Platt, Eds., ACS Symposium Series, No. 932, 2005.

12. Guilbert, B.; Davis, N. J.; Pearce, M.; Aplin, R. T. Tetrahedron: Asymmetry, 1994, 5, 2163-2178.

13. Chang, C.-W. T.; Hui, Y.; Elchert, B.; Wang, J.; Li, J.; Rai, R. Org. Lett. 2002, 4, 4603-4606.

14. Lou, B. in Preparative Carbohydrate Chemistry, Hanessian, S. Ed., Marcel Dekker, Inc. New York, 1997, p389-412. 
15. Chang, C.-W. T. unpublished result.

16. Dasgupta, F; Garegg, P. J. Synthesis 1994, 1121-1123.

17. Radhakrishnan, K. V.; Sajisha, V. S. Synlett. 2005, 997-999. 